JOURNAL OF

SYMPLECTIC GEOMETRY

Volume 3, Number 2, 221-311, 2005

\title{
REIDEMEISTER TORSION IN FLOER-NOVIKOV THEORY AND COUNTING PSEUDO-HOLOMORPHIC TORI, I
}

\author{
YI-JEN LEE
}

This is the first part of an article in two parts, which builds the foundation of a Floer-theoretic invariant, $\boldsymbol{I}_{\mathrm{F}}$.

The Floer homology can be trivial in many variants of the Floer theory; it is therefore interesting to consider more refined invariants of the Floer complex. We consider one such instance - the Reidemeister torsion $\boldsymbol{\tau}_{\mathrm{F}}$ of the Floer-Novikov complex of (possibly non-Hamiltonian) symplectomorphisms. $\boldsymbol{\tau}_{\mathrm{F}}$ turns out not to be invariant under Hamiltonian isotopies, but this failure may be fixed by introducing certain "correction term": We define a Floer-theoretic zeta function $\zeta_{\mathrm{F}}$, by counting perturbed pseudo-holomorphic tori in a way very similar to the genus 1 Gromov invariant. The main result of this article states that under suitable monotonicity conditions, the product $\boldsymbol{I}_{\mathrm{F}}:=\boldsymbol{\tau}_{\mathrm{F}} \boldsymbol{\zeta}_{\mathrm{F}}$ is invariant under Hamiltonian isotopies. In fact, $\boldsymbol{I}_{\mathrm{F}}$ is invariant under general symplectic isotopies when the underlying symplectic manifold $\boldsymbol{M}$ is monotone.

Because the torsion invariant we consider is not a homotopy invariant, the continuation method used in typical invariance proofs of Floer theory does not apply; instead, the detailed bifurcation analysis is worked out. This is the first time such analysis appears in the Floer theory literature in its entirety.

Applications of $\boldsymbol{I}_{\mathrm{F}}$, and the construction of $\boldsymbol{I}_{\mathrm{F}}$ in different versions of Floer theories are discussed in sequels to this article [Y.-J.L.].

\section{Contents}

1. Introduction

1.1. Background and motivations $\quad 222$

1.2. Bifurcation analysis in Floer theories 227

1.3. Notation and conventions 233 
2. Statements of main results 234

2.1. The Floer theory package. 234

2.2. Constructing $\boldsymbol{I}_{\mathbf{F}}$ : the algebraic framework. $\quad 239$

2.3. Statement of the main results 243

3. Floer theory of symplectomorphisms $\quad 246$

3.1. Setup and basics. 247

3.2. Structure of the moduli spaces: $\mathcal{P}, \mathcal{M}_{P}$. 252

3.3. Structure of the moduli spaces: $\mathcal{M}_{O}$. 256

4. Main theorems from a general invariance theorem 263

4.1. Proof of Theorem 2.3.3, assuming Theorem 4.1.1. 263

4.2. A fundamental example. 264

4.3. Regular homotopy of Floer systems 266

4.4. Invariance from RHFS 273

5. Near a minimally degenerate critical point $y \quad 280$

5.1. A decay estimate for the flows. 280

5.2. Fredholm theory and structure theorem for $\mathcal{M}_{P}(x, y)$. 286

5.3. Estimates for the new critical points $y_{+}, y_{-}$. 293

6. Existence of admissible $(J, X)$-homotopies $\quad 295$

6.1. Structure of parameterized moduli spaces. 295

6.2. From a CHFS to an admissible homotopy. 301

References 308

\section{Introduction}

This is the first of a series of papers dealing with torsion invariants in Floer theories. In this paper and its companion, Part II [27], we concentrate on establishing the foundation and invariance of the torsion invariant $I_{\mathrm{F}}$, and thus our main purpose here is to develop a general method for proving such foundational results. Examples, applications, and adaptations to various other versions of Floer theories will be discussed in subsequential papers (e.g, $[29-31])$.

Part I contains the construction of the proposed invariant, the main framework of the invariance proof, and the structure theorems for the relevant moduli spaces. The heavy analysis required for the proof of expected bifurcation behavior is postponed to Part II.

1.1. Background and motivations. The two original versions of symplectic Floer homologies have been initially introduced as tools for proving the Arnold conjecture, which gives a lower bound on the number of fixed points of a Hamiltonian symplectomorphism by the total betti number of 
the symplectic manifold $[\mathbf{1 1}, \mathbf{1 2}]$. Very roughly speaking, the Floer homology is the homology of a "Morse function" (the action functional) on an infinite-dimensional space. In the first version (the "absolute" version), this infinite-dimensional space is the free loop space of the compact symplectic manifold $(M, \omega)$. In the second version (the "relative" version), it is the space of paths ending at two transversely intersecting Lagrangian submanifolds in $M$.

To make the above heuristics work, it actually requires highly nontrivial transversality and compactness results for the relevant moduli spaces, which make use of the assumptions of monotonicity or $\pi_{2}=0$ in Floer's original papers. These assumptions have been subsequently weakened through the efforts of many people; recently, it has been completely removed in the absolute version via the virtual moduli technique by several groups of people, see for example $[\mathbf{1 7}, \mathbf{3 3}, \mathbf{3 8}, \mathbf{3 9}]$. For the relative version, traditionally the success has been more limited, though the recent paper [18] is able to deal with fairly general settings, whose implication includes the general version of Arnold conjecture proved in $[\mathbf{1 7}, \mathbf{3 3}]$.

The basic strategy in these works is the same as Floer's, namely proving the invariance of the Floer homology under Hamiltonian isotopies. This enables one to compute the Floer homology at a small, $t$-independent Hamiltonian, where $H F_{*}(M)=H_{*}(M)$, the usual homology of the symplectic manifold.

Floer's proof guarantees the existence of fixed points for any Hamiltonian symplectomorphism of $M$, because for closed $M, H_{*}(M)$ is never trivial. This is, however, no longer true in many variants of Floer theory. Here are some examples.

\subsubsection{Examples of vanishing Floer homology.}

Example A. (Symplectic manifolds with boundary) There are various ways of defining Floer homologies for symplectic manifolds with contact-type boundaries $[\mathbf{4}, \mathbf{4 6}]$. The Floer homology may be trivial in this situation. For example, $H F_{*}\left(D^{2}\right)=0$ according to Viterbo's definition.

Example B. (Space of loops in a non-trivial homotopy class) To find non-contractible Hamiltonian orbits, one might use the space of noncontractible loops (in a fixed homotopy class) to define Floer homology. However, this version of Floer homology vanishes by its invariance under Hamiltonian isotopies, because for small Hamiltonians, there is obviously no closed orbit $[\mathbf{3}]$.

Example C. (Floer theory of Lagrangian intersections) There are many examples where the relative version - the Lagrangian intersection Floer homology vanishes. For example, the Floer homology of compact Lagrangian submanifolds $L \subset \mathbb{C}^{n}$ vanishes once it is defined and invariant under Hamiltonian isotopies, because it is easy to find Hamiltonian isotopies 
disengaging one from the other. According to [18], such $L$ can be any simplyconnected Lagrangian submanifold in $\mathbb{C}^{n}$ for $n>2$.

Example D. (Twisted versions of Floer theories) This is our prime example. The Floer theory considered in this article will always be the twisted version.

The action functional is in general not globally defined (the usual construction only defines a closed 1-form on the loop space, which is the differential of a real-valued action function only in special cases). Therefore, one often needs to consider twisted versions of Floer homology, modeling on the Morse theory of closed 1-forms introduced by Novikov. A typical example is the Floer homology of a non-Hamiltonian symplectomorphism $f$.

For any symplectic isotopy connecting the identity with a non-Hamiltonian symplectomorphism $f$, Le-Ono [32] defined such a twisted version of Floer homology and showed, following Floer's strategy, that when $M$ is monotone, its total betti number is equal to the total betti number of the Novikov homology $H N\left(M, \theta_{f}\right)$. (Le-Ono actually had a slightly weaker assumption on $M$.) $\theta_{f} \in H^{1}(M)$ above is the "flux" or "Calabi invariant" of $f$, and the Novikov homology $H N\left(M, \theta_{f}\right)$ is a twisted version of Morse homology.

Le-Ono's result guarantees the existence of symplectic fixed points in many cases; however, there are also many examples where this version of Floer homology vanishes. The reason is that the "twisting" procedure often reduces the rank of the homology: different twisted versions of homologies correspond to homologies of different coverings, and the larger the covering group is, the smaller the rank of the corresponding homology is.

1.1.2. Torsion invariants in Floer and Morse theories. Naturally, one is interested in more refined invariants of the Floer complex when the Floer homology vanishes.

It is known that the finite-dimensional Morse theory captures much more than homological informations of the underlying manifold; in fact, Cohen-Jones-Segal showed that the Morse theory recovers the entire homeomorphism type of the underlying manifold [5]. One therefore, expects that Floer theory similarly has much more to offer than merely the Floer homology. The path from Morse theory to Floer theory is strewn with heavy technical difficulties (see Section 1.2 below for some discussion); here are, however, some instances of progress in this direction, in the context of symplectic Floer theories:

Fukaya proposed to study the $A_{\infty}$ structure of Floer theory which will capture the full rational homotopy type of the Floer complex. In $[\mathbf{1 6}]$, Fukaya and $\mathrm{Oh}$ showed that the $A_{\infty}$ category of the Lagrangian intersection Floer theory for cotangent bundles is equivalent to the $A_{\infty}$ category of the Morse theory. 
As the first non-homotopy invariant historically, the Reidemeister torsion is the obvious next candidate to consider. In 1994, Fukaya proposed studying torsion and even higher torsions in symplectic Floer theories. In [15], He sketched the definition of the Whitehead torsions for symplectomorphisms and Lagrangian intersections, claimed that they are invariant under Hamiltonian isotopies, and conjectured that the Floer homology together with the torsion give complete obstructions of Hamiltonian isotopying a symplectomorphism to one without fixed points, or a Lagrangian submanifold to one without intersection with another fixed Lagrangian submanifold (the "symplectic s-cobordism conjecture"). There were, however, no details.

More recently, rigorous works in this direction were done by EliashbergGromov [9] and Sullivan [43]. However, to sidestep the substantial technical difficulties in the invariance proof (see Section 1.2, II.1 for more discussion), these results require restrictive assumptions or ad hoc methods: EliashbergGromov consider only graphical Lagrangian submanifolds in cotangent bundles, so that it may be reduced to purely finite-dimensional method of generating functions. Sullivan made several restrictive assumptions to ensure that the action functional is globally defined, which do not hold in general (cf. discussion in Example D earlier). He also used a stabilization trick to sidestep the analysis of death-birth bifurcations, which applies only to the untwisted version of Lagrangian intersection Floer theory. In both $[\mathbf{9}]$ and $[\mathbf{4 3}]$, the Lagrangian submanifolds have to be non-compact for their versions of torsion to be non-trivial. (Compare with [30], where an adaptation of this article gives torsion invariants that are often non-trivial for compact Lagrangian submanifolds).

One goal of this article is to provide, once and for all, a direct and general invariance proof that should work for any version of Floer theory, by overcoming the analytical difficulties. In this paper, we consider a general version of the Floer theory of symplectomorphisms (cf. Section 3.1), which covers Examples B and D provided earlier. Analogous results for the versions of Floer theories in Examples $\mathrm{A}$ and $\mathrm{C}$ can be obtained by simple adaptations of this paper (see e.g., $[\mathbf{2 9}, \mathbf{3 0}]$ ). We study the Reidemeister torsion of the Floer complex, denoted $\tau_{\mathrm{F}}$. The torsion we use is an abelian (and hence weaker) version of the Whitehead torsion. On the other hand, since this definition uses a standard set of bases singled out by the Morse-theoretic context, it is also in a sense more refined. See Section 2.1 for the precise definition.

However, $\tau_{\mathrm{F}}$ is not an invariant for the twisted versions of Floer theories in Example D. Nevertheless, we find that one may construct an invariant by taking into account (perturbed) pseudo-holomorphic tori. More precisely, we define the product

$$
I_{\mathrm{F}}:=\tau_{\mathrm{F}} \zeta_{\mathrm{F}}
$$


where the "zeta function" $\zeta_{\mathrm{F}}$ is a generating series counting perturbed pseudo-holomorphic tori. Our main result is that $I_{F}$ is a symplectic invariant.

In fact, this result should be viewed as an infinite-dimensional analog of our previous results on the torsion invariants of Morse 1-forms [2123]. Given a Morse 1-form $\theta$ on a closed, finite-dimensional manifold $M$, we considered the dynamics of the flow generated by the vector field dual to $\theta$. The Reidemeister torsion $\tau$ of the associated Morse-Novikov complex counts flow lines ending in critical points in a sense; whereas the dynamical zeta function $\zeta$ counts the closed orbits. We showed that the product $I=\tau \zeta$ is independent of the metric or $\theta$, though neither $\tau$ nor $\zeta$ alone is invariant. Actually, $I$ is equivalent to the combinatorially defined Reidemeister torsion of the manifold $M$. In keeping with the picture of the Floer theory as an infinite-dimensional Morse theory, the flow lines in the loop space correspond to perturbed pseudo-holomorphic curves. Thus, $\tau_{\mathrm{F}}$ and $\zeta_{\mathrm{F}}$ above are, respectively, the infinite-dimensional analogs of the Reidemeister torsion of the Morse-Novikov complex and the dynamical zeta function.

1.1.3. Relation with Gromov theory. Due to the nature of the definition of $I_{\mathrm{F}}$ as a product, one may either regard it as a refinement of Floer homologies, or as a counting invariant of pseudo-holomorphic curves. These two perspectives lead to different types of applications. One incentive for our choice of the abelian version of torsion over the non-abelian Whitehead torsion is so that $I_{\mathrm{F}}$ counts perturbed pseudo-holomorphic curves with homology class, which is more in keeping with the usual definition of curvecounting invariants (Gromov invariants). In fact, $I_{\mathrm{F}}$ much resembles the Gromov-Taubes invariant. The perturbed pseudo-holomorphic tori here has the interpretation as the perturbed pseudo-holomorphic sections of the symplectic mapping tori $(M \times[0,1] /(x, 0) \sim(f(x), 1)) \times S^{1}$, and the definition of $\zeta_{\mathrm{F}}$ is very similar to the zeta function introduced by Ionel-Parker in [25], which computes an averaged version of the genus 1 Gromov invariant of symplectic mapping tori. A fascinating problem is to better understand the precise relation between $I_{\mathrm{F}}$ and genus 1 Gromov invariants: such a relation would provide a link between Floer theory and Gromov theory, which may be applied in both directions. Some simplest cases of this relation are discussed for two variants of $I_{\mathrm{F}}$ in $[\mathbf{3 0}]$, which lead to interesting results in symplectic topology that are beyond the reach of either Floer theory or Gromov theory alone.

More generally, "physical reasoning" leads one to expect a Floertheoretic interpretation of genus 1 Gromov invariants along the line of our construction of $I_{\mathrm{F}}$. Philosophically, mirror symmetry is a correspondence between symplectic (A-model) and complex (B-model) geometries. Since the complex side is typically easier to compute, mirror symmetry produces many 
interesting conjectural formulae for the curve-counting invariants on the symplectic side. Recently, much progress has been made in mathematically rigorous formulation and verification of 0-loop mirror symmetry, but the higher genera case remains little understood. In their famous paper in 1993, the four physicists Bershadsky, Cecotti, Ooguri, Vafa extended mirror symmetry to higher genera curves [2]. At the 1-loop level, this says that the generating series of genus 1 Gromov-Witten invariants from the A-model side should correspond to certain holomorphic analytic torsion from the B-model side. On the other hand, Kontsevich formulated the 0-loop mirror symmetry as an equivalence between the Fukaya-Floer category from the A-model side, and the category of coherent sheaves on the B-model side. In particular, the Floer cohomologies should correspond to certain sheaf cohomologies on the B-model side. A naive generalization of Kontsevich's proposal leads one to expect higher-loop mirror symmetry as an equivalence of secondary invariants on symplectic and complex sides. In particular,

generating series of genus 1 Gromov invariants "=" Floer-theoretic torsion $\zeta_{\mathrm{F}}$ "=" torsion on symplectic side;

holomorphic analytic torsion "=" torsion on complex side.

Our construction using $\zeta_{\mathrm{F}}$ to "correct" the Reidemeister torsion of the Floer complex also has a better-known analog on the complex side: log of the holomorphic analytic torsion can be viewed as the "correction term" to the $L^{2}$-metric on the determinant line bundle of sheaf cohomologies, and the combination of the two defines the invariant Quillen metric. (This above observation was due to Fukaya [18].)

1.1.4. Higher torsions in Floer theories. In [24], Igusa defined higher Franz-Reidemeister torsions via parametrized Morse theory. The bifurcation analysis carried out in this paper enables one to lift Igusa's work to the Floer-theoretic setting. In principle, these Floer-theoretic higher torsions may be useful for investigating homotopy groups of the symplectomorphism groups. We hope to return to this subject in the future.

1.2. Bifurcation analysis in Floer theories. Our method of invariance proof is very different from the traditional one.

Hitherto, the only available tool for invariance proof in Floer theory is the "continuation method" (cf. e.g., $[\mathbf{1 2}]$ ), which relies on the construction of chain homotopy equivalences between Floer complexes. The drawback of this method is that it is only useful for proving the invariance of homotopy invariants such as homology or $A_{\infty}$ categeory. It does not apply for nonhomotopy invariants such as the Reidemeister torsion considered in this paper.

A natural and straightforward approach, which would apply in more general situations, is direct bifurcation analysis. Namely, consider a generic 
1-parameter family of Floer complexes, classify the possible bifurcations, and study how the proposed invariant change at these bifurcations.

In his first paper on Floer homologies [11], Floer gave a very brief outline of this approach in the case when the action functional is globally defined. The details were, however, largely missing, and this method was subsequently superceded by the continuation method mentioned earlier. During the long gestation period of the present article, some recent papers following Floer's original approach have appearred, see for example, $[\mathbf{7}, \mathbf{4 3}]$. As noted earlier, these papers, including Floer's, rely on ad hoc methods peculiar to the special cases considered. (In particular, they do not apply to the situation considered here).

In this article, we present a fairly general invariance proof which carries out this direct approach in its entirety. This proof is amenable to adaptations to other versions of Floer theories (see e.g., $[\mathbf{3 0}]$ ). In addition to openning the door for the study of other more refined Floertheoretic invariants, it also offers a viable alternative to the conventional continuation method in more intricate variants of Floer homology, as [7] demonstrates.

Since we work in the general Morse-Novikov situation, where the action functional may not be globally defined, there are several important new features which did not appear in previous works by Floer and other authors, making the analysis substantially harder than the situation previously considered. Further remarks follow in Sections 1.2.1 and 1.2.2.

1.2.1. Finite-dimensional model: the Morse-theoretic picture. It is helpful to first understand the simpler case of Morse theory on a finitedimensional manifold.

Given a generic 1-parameter family of Morse functions, there are two basic types of bifurcations: a "handle-slide", namely a flow line between two nondegenerate critical points of the same index, or a "death-birth", namely two critical points cancelling each other.

Moreover, it is not hard to understand how the Morse complexes change at a bifurcation point, thanks to the geometric interpretation of flow lines as intersection points (of ascending/descending manifolds, or in the case of closed orbits, as the fixed points of a local Poincaré return map).

For real-valued Morse theory, the Morse complex changes by an elementary transformation at a handle-slide, and at a death-birth, the Morse complex changes by an expansion/collapse modulo elementary transformations. (see Remark 4.4.3(b) for terminologies) This shows that not only the homotopy type of the Morse complex is invariant, but its simple-homotopy type is invariant.

For Morse theory of closed 1-forms, the bifurcation behavior is more complicated, but has been partially worked out in $[\mathbf{2 1}]$. We list some major 
differences between the real-valued Morse theory and the Morse-Novikov theory of a non-exact closed 1-form below.

(1) Unlike the case with real-valued Morse theory, in this case the moduli spaces of flows between two fixed critical points are non-compact. To obtain a well-defined Morse complex in this setting, one needs to work with Novikov coefficients. Of crucial importance here is a filtration, which depends on the cohomology class of the Morse 1-form, modulo rescaling by positive numbers. (The truncated moduli space of flows with an upper bound on energy is compact). The 1-parameter family of Morse 1-forms $\left\{\theta_{\lambda}\right\}_{\lambda \in \Lambda}$ used for the invariance proof should have the same filtration, namely, their cohomology classes should be "co-directional" in the sense that $\left[\theta_{\lambda}\right]=\alpha_{\lambda}[\theta]$ for a fixed $[\theta] \in H^{1}$, and a family of non-negative numbers $\left\{\alpha_{\lambda}\right\}_{\lambda \in \Lambda}$.

(2) The flow lines may form closed orbits. At a bifurcation point, infinitely many new isolated flow lines between critical points, or closed orbits, may be generated by gluing arbitrarily many flow lines simultaneously.

(3) There can be infinitely many handle-slides in a family of MorseNovikov theory parametrized by a compact interval $\Lambda \subset \mathbb{R}$. As the Morse complex is undefined on a possibly dense subset of $\Lambda$, even the notion of the "change" of the Morse complex at a bifurcation point requires careful definition.

At a handle-slide bifurcation where a flow line starts and ends at the same critical point (called "type II handle-slide" in this article), the higher order (in terms of energy filtration) bifurcation behavior is not understood. In [21], Hutchings side-stepped this problem by considering the induced flow on finite-cyclic coverings of the manifold, using the relation between the torsion of $M$ and of its finite-cyclic coverings to reduce the higher order problem to the low order problem. We shall follow his approach.

1.2.2. From Morse theory to Floer theory. While Morse theory gives a nice model for Floer theory, there are several important differences. For example:

(1) The grading of a Floer complex often takes values not in the group $\mathbb{Z}$, but rather in $\mathbb{Z} / N \mathbb{Z}$ for some $N \in \mathbb{Z}^{+}$. In order for the Reidemeister torsion to be well-defined, $N$ has to be even. It is not hard to see that this is the case for the version of Floer theory considered in this article.

(2) The geometric interpretation of flow lines mentioned in Section 1.2.1 is no longer available in the infinite-dimensional context of Floer theory. Instead, the spaces of flow lines need to be described as moduli spaces of certain elliptic PDEs. The verification of the bifurcation behaviors predicted by Morse theory requires detailed understanding 
of the behaviors of moduli spaces under bifurcation, which relies on certain gluing theorems. Proving these gluing theorems constitutes the major difficulty in this direct approach of invariance proof. (In comparison, the traditional continuation method only requires certain characteristic numbers of the relevant moduli spaces to be welldefined). Part II will deal with this problem.

(3) While a Morse function admits rather flexible perturbations, only very restrictive perturbations to a Floer theory make good moduli problems. Consequently, some trivial facts in Morse theory become highly non-trivial in a Floer theory. As a well-known instance, the transversality proofs in Floer theory can be rather involved, unlike the case of Morse theory. Two instances relevant to this article are:

(a) To apply Hutchings's argument for type II handle-slide bifurcation, one needs non-equivariant perturbations to the induced flow on finite-cyclic covers of the loop space (see Section 4.4.5 for a more precise statement). In the Floer theory context, it is highly non-trivial to construct such perturbations satsifying the desired properties. Unless one restricts to special classes of symplectic manifolds, the perturbation will be non-local, and new arguments are required to establish the usual transversality and compactness properties of the moduli spaces (see Section 6 of Part II).

(b) As explained in item 1 of Section 1.2.1 earlier, the 1-parameter family of Morse 1-forms used in the invariance proof must be "codirectional". Nonetheless, the finite-dimensional invariant $I$ is independent of the Morse 1-form used for its definition, regardless of its cohomology class. This is because any Morse 1form may be connected to an exact one via a path of co-directional closed 1-forms. In Floer theory, it is often difficult to find a path which both satisfies the co-direction condition and makes a good moduli problem. In our Floer-theoretic context, the analog of the Morse 1-form is the "action 1-form" $y_{X}$. Perturbing the symplectic vector field $X$ by Hamiltonian vector fields does not change the cohomology class $\left[y_{X}\right]$, but perturbing by a general symplectic vector field does. While one expects $I_{\mathrm{F}}$ to be invariant under Hamiltonian isotopies, whether it is invariant under general symplectic isotopies depends on whether one may find a symplectic vector field $X_{0}$ such that $\left[y_{X_{0}}\right]=0$. The existence of such $X_{0}$ is guaranteed by imposing strong monotonicity conditions on the symplectic manifold; see Theorem 2.3.3(b) for a precise statement. It is not known whether $I_{\mathrm{F}}$ is invariant under symplectic isotopies in general. 
1.2.3. Adaptability of the method. We now briefly indicate which portion of the arguments in this article is independent of the specific Floer theory considered. By the "Floer theory", we mean one which satisfies the properties outlined in Section 2.1 below.

The following depend on the specific Floer theory: part of the transversality and compactness arguments, such as the structure theorems of the moduli spaces proven in Section 3, and the arguments in Section 6, and the construction of non-equivariant perturbations in Section 6.3 of Part II; the orientability of moduli spaces, as discussed in Section 7.2 of Part II.

The following are universal for all Floer theories: the main framework of proof for the general invariance theorem, Theorem 4.1.1 below; the analysis for moduli space of flows ending at a degenerate critical point contained in Section 5; the main outline of the proofs of gluing theorems in Sections 2-5 in Part II applies to any version of Floer theory; the coherentness of orientations of moduli spaces discussed in Section 7.3 of Part II, which rely on linear versions of gluing theorems. The estimates in the proofs of these gluing theorems require the following additional conditions, which hold for most of the existent Floer theories:

- Recall that the Floer theory is constructed from the formal flow of a (densely defined) vector field $\mathcal{V}$ on a Banach manifold $\mathcal{C}$ modeling on a function space. In local coordinates, we need $\mathcal{V}$ to depend only on the function itself, not its higher derivatives, at least in a neighborhood of the degenerate critical points.

In symplectic Floer theories, this condition holds by the assumption of the degenerate critical points being in "standard d-b neighborhoods" (cf. Section 5.3). In gauge theories, it follows directly from the defining formula for $\mathcal{V}$ that this condition holds anywhere on $\mathcal{C}$.

- We use the Sobolev embedding theorem to obtain $C_{0}$ estimates from $L^{p}$-estimates on several occasions. The applicability of Sobolev embedding depends on the dimension $1+l$, when $T C$ is a space of functions/sections over an $l$-dimensional manifold. In this article, as well as in other symplectic Floer theories, $l=1$. In a typical gauge theory, $l=3$. In this case, we need to require $p \geq 4$ for these specific estimates to work.

We shall also consider in [30] an equivariant version of Floer theory, that does not entirely fit in the framework of Section 2.1.2. Nevertheless, a minor modification of the methods of this article still serves the purpose.

1.2.4. Outline of part I. In Section 2, we summarize the abstract framework for defining the invariant $I_{\mathrm{F}}$, and give precise statements of the main results. The concrete Floer theory considered in this article is set up in Section 3, where structure theorems of the relevant moduli spaces needed 
for the construction of $I_{\mathrm{F}}$ are established. In Section 4, we state a general invariance theorem, Theorem 4.1.1, and show how it implies the theorems stated in Section 2. Based on the finite-dimensional Morse theoretic picture, we summarize the expected bifurcation behavior of a generic 1-parameter family of Floer systems into the notion of a "regular homotopy of Floer systems" (RHFS). Following [21], it is shown that the existence of an RHFS with the extra property (NEP) implies the general invariance theorem 4.1.1. Part II and the rest of Part I are devoted to establish the existence of such an RHFS.

Since a degenerate critical point appears at a death-birth bifurcation, we need to describe the structure of the moduli spaces of flows ending at such degenerate critical points. This is done in Section 5. Section 6 introduces the notion of an "admissible $(J, X)$-homotopy", which is equipped with most of the properties of an RHFS. We then establish the existence of an admissible ( $J, X)$-homotopy.

Based on a series of hard gluing theorems, Part II will show that an admissible $(J, X)$-homotopy also possess the remaining properties of an RHFS. There, the issue of orientation will also be addressed, thus concluding the proof of Theorem 4.1.1. See Section 1 of Part II for a summary of these results, and an outline of the methods of proof.

The main technical components of Part I are contained in different sections as follows:

Section 3.3, in which we establish transversality for moduli spaces of closed orbits in homology class $A, \hat{\mathcal{M}}_{O}^{0}(A)$, simultaneously for all $A$. This depends on an adaptation of Taubes's argument in [44], which translates the problem of transversality for multiple covers into the simultaneous surjectivity of a sequence of differential operators over simple orbits. The knowledge of the kernels and cokernels of these differential operators as representation spaces of finite-cyclic groups helps to solve the latter problem.

Section 5, in which we establish the polynomial decay of flows ending at a degenerate critical point, and the Fredholm theory for the moduli of such flows. The decay estimates employs center manifold theory for flows on Hilbert manifolds, modeling on the discussion in [36]. To find the suitable Fredholm framework, the appropriate spaces for the domain and range of the deformation operator turn out to be somewhat complicated, due to the following multiple constraints:

(i) the deformation operator must be Fredholm between these spaces;

(ii) the domain must be big enough to contain the moduli space considered; 
(iii) the non-linear part of the PDE must satisfy the expected quadratic bound.

They are polynomially weighted Sobolev spaces, but the weights on the "transversal directions" and the "longitudinal direction" are different. The rationale for the choice is that the deformation operator is modeled on the operator $d / d s+$ $C$, where $C$ is a constant that is non-zero for the transversal directions, and zero for the longitudinal directions.

Section 6.2, where we show how to perturb a 1-parameter family of Floer systems into an admissible $(J, X)$-homotopy, based on a refinement of the standard transversality arguments.

Due to the non-compactness of moduli spaces (cf. e.g., Section 1.2.1 items 2 and 3), careful filtration arguments are frequently called for throughout the length of this article (see in particular Sections 3.3 and 6.2).

1.3. Notation and conventions. The following notation and conventions are adopted in both parts of this article. References to section or equation numbers in part II will be denoted as II.*. We suggest the reader to first browse through this subsection and to return later for reference of the notations.

- $C, C^{\prime}, C_{i}$, etc., usually denote positive constants depending on the context. $\varepsilon, \varepsilon^{\prime}$ usually denote small positive numbers. In contrast, the plain $\epsilon$ usually denotes a fixed parameter; for example, in Sections 3 and 5 it parameterizes the weight in the exponentially weighted Sobolev spaces; in II.2-II.5 as it denotes a fixed small number associated with the choice of partition of $\Theta$.

- $\Pi_{V}$ in general denotes a projection. It denotes the projection to the space $V$ if $V$ is a space; or to the direction of $V$ if $V$ is a vector.

- $S_{T}^{1}=\mathbb{R} / T \mathbb{Z}$ denotes the circle of length $T$.

- The top exterior power of a vector space $V$ is denoted by $\operatorname{det} V$.

- $\|\xi\|_{p, k}$ denotes the Sobolev norm obtained by summing $L^{p}$ norms of derivatives of $\xi$ up to order $k . L_{k}^{p}$ denotes the associated Sobolev space. Throughout this article, $p$ is an integer $p>2$.

- $C_{\epsilon}^{\infty}=C_{\epsilon} \subset C^{\infty}$ denotes the Banach space endowed with the $\|\cdot\|_{\epsilon^{-}}$ norm defined in [10].

- Let $L$ be any of the norms used in this article, and $F$ be an Euclidean/Hermitian vector bundle over $M . L(M)$ denotes the space of functions on the manifold $M$ with finite $L$-norm. $L(M ; F)=L(F)$ denotes the space of sections of $F$ with finite $L$-norm.

- In this paper, the inner product $\langle\cdot, \cdot\rangle_{2}$, and the norms $\|\cdot\|$ are usually for functions or sections over the cylinder or torus $\Theta=\left\{(s, t) \mid t \in S^{1}\right\}$, $s \in \mathbb{R}$ or $S_{T}^{1}$. We will occasionally encounter restrictions of these functions/sections to a circle of fixed $s$ in $\Theta$. The inner product or norms of such restrictions are denoted by $\langle\cdot, \cdot\rangle_{2, t},\|\cdot\|_{p, t}$, etc. 
- Let $\xi$ be a section over $\Theta$. $\partial_{s}, \partial_{t}$ denote the covariant derivative with respect to a natural connection, for example, that induced from the Levi-Civita connection. We also often use the short hand $\dot{\xi}:=\partial_{t} \xi$; $\xi^{\prime}:=\partial_{s} \xi$.

- Many functions in this paper depend on both the position of a point in the symplectic manifold $M$, and other variables such as $s, t, \lambda$. However, the notation $\nabla$ is always taken to mean the gradient as a function of $M$, holding other variables constant. Derivatives in the directions of $s, t, \lambda$ are denoted $\partial_{s}, \partial_{t}, \partial_{\lambda}$, respectively.

- Let $R$ be a commutative ring. $Q(R)$ denotes the total ring of fractions (also called quotient ring) of $R$. Namely, the localization at all nonzero divisors of $R$.

- Let $V_{1}, V_{2}$ be two isomorphic oriented spaces. Then $V_{1} / V_{2} \in\{1,-1\}$ denotes the relative sign of them. If $V_{1} / V_{2}=-1$, we write $V_{1}=-V_{2}$. If $V_{1}$ is one-dimensional and $a$ is a non-trivial element of $V_{1}$, then $[a] / V_{1}$ denotes the sign of $a$ with respect to the orientation of $V_{1}$. $\operatorname{sign}(u):=u /|u|$ for $u \in \mathbb{R}$.

- We follow the convention of calling a point in a moduli space (Zariski) smooth or non-degenerate if the relevant deformation operator at that point is surjective. A moduli space is said to be (Zariski) smooth or non-degenerate if it consists of smooth points.

- Following a well-known convention, a vector field on a Banach space refers to one that is only densely defined.

More notation and conventions will be introduced along the way in later sections.

\section{Statements of main results}

Some preliminaries are required for the precise statements of our results.

2.1. The Floer theory package. As our construction of Floer-theoretic torsions applies to many versions of Floer theories, we give here a general outline of the abstract Floer-theoretic framework needed for this construction. The concrete content of the specific version of Floer theory considered in this article will be described in Section 3. The main difference between the following discussion and those in the existent literature consists of the requirement of an absolute $\mathbb{Z} / 2 \mathbb{Z}$-grading, the attention to the moduli spaces of closed orbits, and the emphasis on "grading-compatible" orientation for the moduli spaces of closed orbits.

2.1.1. Basic ingredients: $\left(\mathcal{C}, \mathfrak{H}\right.$, ind $\left.; y_{\chi}, \mathcal{V}_{\chi}\right)$. In a typical Floer-Novikov theory, one has an (infinite-dimensional) Banach manifold $\mathcal{C}$, and a closed 1 -form $y_{\chi}$ on $\mathcal{C}$, which may depend on certain parameter $\chi$. Together with an $L^{2}$-metric on $\mathcal{C}$ (also possibly dependent on $\chi$ ), $y_{\chi}$ determines a (densely 
defined) dual vector field $\mathcal{V}_{\chi}$ on $\mathcal{C}$. This defines a formal flow on $\mathcal{C}$ in the following sense: The moduli space of critical points, $\mathcal{P}_{\chi} \subset \mathcal{C}$ is defined as the zero locus of $\mathcal{V}_{\chi}$. A flow line $u$ is a solution to the elliptic PDE, $\partial_{s} u+\mathcal{V}_{\chi}(u)=0$, where $s \in \mathbb{R}$ or $S^{1}$. It is called a closed orbit when $s \in S_{T}^{1}$, for some $T>0$, and $T$ is said to be the period of the closed orbit. When $s \in \mathbb{R}$ and $u(s)$ approaches critical points as $s \rightarrow \pm \infty$, it is called a connecting flow line. The moduli spaces of non-constant closed orbits and the moduli spaces of connecting flow lines will be denoted, respectively, by $\mathcal{M}_{O}(\chi)$ and $\mathcal{M}_{P}(\chi)$. A closed orbit is typically written as either $u$ or $(T, u)$, when one wants to emphasize its period.

In order for the Floer-theoretic torsion to be defined, we consider Floer theories endowed with an absolute $\mathbb{Z} / 2 \mathbb{Z}$-grading: Let $\mathcal{P}_{\chi, \text { ndg }} \subset \mathcal{P}_{\chi}$ be the set of non-degenerate elements. There is a map

$$
\text { ind : } \mathcal{P}_{\chi, \text { ndg }} \rightarrow \mathbb{Z} / 2 \mathbb{Z} \quad \forall \chi
$$

defined by the spectral flow from certain standard operator to the linearization of $\mathcal{V}_{\chi}$ at $x \in \mathcal{P}_{\chi, \text { ndg }}$, denoted $A_{x}$.

Let $\tilde{\mathcal{C}}$ denote the regular covering of $\mathcal{C}$ with an abelian covering group $\mathfrak{H}$ and monodromy homomorphism im $: \pi_{1}(\mathcal{C}) \rightarrow \mathfrak{H}$.

Notation. We shall frequently use the following notation for elements in a covering space. Let $\gamma_{0} \in \mathcal{C}$ be a base point. Identify $\tilde{\mathcal{C}}$ with the set $\tilde{\mathrm{C}}^{\gamma_{0}}$ of equivalence classes $(x,[w])$, where $x \in \mathcal{C}, \mu$ is a map $[0,1] \rightarrow \mathcal{C}$, $\mu(0)=\gamma_{0} ; \mu(1)=x$, and two pairs $(x,[w]),\left(x^{\prime},\left[w^{\prime}\right]\right)$ are equivalent iff $x=x^{\prime}$, and $\operatorname{im}\left[\mu-\mu^{\prime}\right]=0$.

We consider Floer theories in which $\mathfrak{H}$ can be chosen so that for any $\chi$,

- $y_{\chi}$ lifts to an exact form $d \mathcal{A}_{\chi}$. Namely, the cohomology class $\left[y_{\chi}\right]=\underline{\mathrm{im}}^{*}\left\langle y_{\chi}\right\rangle$, where $\left\langle y_{\chi}\right\rangle \in \operatorname{Hom}(\mathfrak{H}, \mathbb{R})$, and $\underline{\mathrm{im}}$ is the abelianization of im.

- The absolute $\mathbb{Z} / 2 \mathbb{Z}$-valued grading lifts to a relative $\mathbb{Z}$-valued grading: Let $\tilde{\mathcal{P}}_{\chi} \subset \tilde{\mathcal{C}}$ denote the lift of $\mathcal{P}_{\chi}$. There is a map gr $: \tilde{\mathcal{P}}_{\chi, \text { ndg }} \times \tilde{\mathcal{P}}_{\chi, \text { ndg }} \rightarrow$ $\mathbb{Z}$, so that for any $(x,[w]),(y,[v]),(z,[r]) \in \tilde{\mathcal{P}}_{\chi}$,

$$
\begin{gathered}
\operatorname{gr}((x,[w]),(y,[v]))+\operatorname{gr}((y,[v]),(z,[r]))=\operatorname{gr}((x,[w]),(z,[r])) ; \\
\operatorname{gr}((x,[w]),(y,[v])) \quad \bmod 2=\operatorname{ind}(x)-\operatorname{ind}(y), \quad \text { and }
\end{gathered}
$$

there is a homomorphism (which we call the SF-homomorphism)

$$
\psi: \mathfrak{H} \rightarrow 2 \mathbb{Z}
$$

with the following property: Let $(x,[w]),\left(x,\left[w^{\prime}\right]\right)=A \cdot(x,[w])$ be different lifts of the same $x \in \mathcal{P}_{\chi}$, where $A \in \mathfrak{H}$ acts by deck transformation. Then

$$
\operatorname{gr}\left(\left(x,\left[w^{\prime}\right]\right),(x,[w])\right)=\psi(A) .
$$


Via index theory, $\psi$ is typically computable from the topology of the underlying manifold and is independent of the parameter $\chi$. However, in a general Floer theory it is only expected to take value in $\mathbb{Z}$ instead of $2 \mathbb{Z}$, as we require here.

Sometimes weighted versions of ind and gr are also needed: For $\sigma, \sigma_{1}, \sigma_{2} \in$ $\mathbb{R}$, ind ${ }^{\sigma}(x)$ and $\operatorname{gr}^{\left(\sigma_{1}, \sigma_{2}\right)}\left(\left(x_{1},\left[w_{1}\right]\right),\left(x_{2},\left[w_{2}\right]\right)\right)$ are defined similarly to ind and gr, but with the role of $A_{x_{*}}$ replaced by $A_{x_{*}}+\sigma_{*}$.

We shall often denote a Floer theory by the five-tuple of its basic ingredients: $\left(\mathcal{C}, \mathfrak{H}\right.$, ind $\left.; y_{\chi}, \mathcal{V}_{\chi}\right)$. Note that the first three items are independent of the parameters, while the last two do.

2.1.2. Variants of moduli spaces. We now introduce some notation and terminologies frequently used in this article. Throughout this subsubsection, we work with a fixed parameter $\chi$. Therefore, though all the moduli spaces below depend on the parameter, we shall omit $\chi$ from the notation.

Let $\mathcal{M}_{P}(x, y)$ denote the moduli space of connecting flow lines starting from the critical point $x$ and ending at the critical point $y$. Given $(x,[w])$, $(y,[v]) \subset \tilde{\mathcal{P}}_{\chi}, \mathcal{M}_{P}((x,[w]),(y,[v])) \subset \mathcal{M}_{P}(x, y)$ denote the subset of elements which lift to a path in $\mathcal{C}$ starting from $(x,[w])$ and ending in $(y,[v])$.

Given $A \in \mathfrak{H}, \mathcal{M}_{O}(A) \subset \mathcal{M}_{O}$ denotes the set of closed orbits with homotopy class in $\mathrm{im}^{-1}(A)$.

The grading of a closed orbit $u \in \mathcal{M}_{O}(A)$ is given by $\psi(A)$. For a connecting flow line $u \in \mathcal{M}_{P}((x,[w]),(y,[v]))$, it is given by $\operatorname{gr}((x,[w])$, $(y,[v]))=k$. Let $\mathcal{M}_{O}^{k+1} \subset \mathcal{M}_{O}, \mathcal{M}_{P}^{k}(x, y) \subset \mathcal{M}_{P}(x, y)$ denote the subset consisting of elements with grading $k$.

Weighted versions of moduli spaces are occasionally needed. Roughly speaking, $\mathcal{M}_{P}^{\left(\sigma_{1}, \sigma_{2}\right)} \subset \mathcal{M}_{P}$ consists of connecting flow lines decaying exponentially at $\pm \infty$ ends, with the exponent $>\sigma_{1}$ in the negative end, and $>-\sigma_{2}$ in the positive end. $\mathcal{M}_{P}^{k,\left(\sigma_{1}, \sigma_{2}\right)}$ consists of such connecting flow lines with the weighted grading $\operatorname{gr}^{\left(\sigma_{1}, \sigma_{2}\right)}=k$.

The energy of an element $u \in \mathcal{M}_{O}(A)$ is given by $-\left\langle y_{\chi}\right\rangle(A)$; for an element $u \in \mathcal{M}_{P}((x,[w]),(y,[v]))$, it is given by $\tilde{\mathcal{A}}_{\chi}(x,[w])-\tilde{\mathcal{A}}_{\chi}(y,[v])$.

We often need truncated versions of moduli spaces: Given $\Re \in \mathbb{R}$, let $\mathcal{M}_{O}(\chi)^{\Re} \subset \mathcal{M}_{O}(\chi), \mathcal{M}_{P}(\chi)^{\Re} \subset \mathcal{M}_{P}(\chi)$ be the subsets consisting of elements with energy $\leq \Re$. Similarly for other variants of $\mathcal{M}_{P}, \mathcal{M}_{O}$.

Note that there is a free $\mathbb{R}$-action on $\mathcal{M}_{P}$ and a semi-free $S^{1}$-action on $\mathcal{M}_{O}$ by translation. The reduced moduli spaces $\hat{\mathcal{M}}_{P}, \hat{\mathcal{M}}_{O}$, are respectively, the quotient spaces of $\mathcal{M}_{P}, \mathcal{M}_{O}$ under this action; similarly for other variants of $\mathcal{M}_{P}$ and $\mathcal{M}_{O}$ introduced above. In particular, $\hat{\mathcal{M}}_{P}^{k}=\mathcal{M}_{P}^{k+1} / \mathbb{R} ; \hat{\mathcal{M}}_{O}^{k}=$ $\mathcal{M}_{O}^{k+1} / S^{1}$. An element in the reduced moduli space is called a reduced flow line. Given $u \in \mathcal{M}_{P}$ or $\mathcal{M}_{O}$, we use $\hat{u}$ to denote the corresponding element in the reduced moduli space; conversely, $u$ is said to be a representative of $\hat{u}$. 
Given an element $u \in \mathcal{M}_{O}$, mult $(u)=\operatorname{mult}(\hat{u})$ denotes the multiplicity of $u$. Namely, the order of the stabilizer at $u$ under the $S^{1}$-action. A closed orbit of multiplicity 1 is said to be simple; otherwise it is said to be a multiple cover.

It is often convenient to identify the reduced moduli spaces with a slice of representatives in the unreduced version. In particular, we often identify $\hat{\mathcal{M}}_{P}$ with the set of centered elements in $\mathcal{M}_{P}$, where an element $u \in \mathcal{M}_{P}((x,[w]),(y,[v]))$ is centered if

$$
2 \tilde{\mathcal{A}}_{\chi}(u(0))=\tilde{\mathcal{A}}_{\chi}(x,[w])-\tilde{\mathcal{A}}_{\chi}(y,[v]) .
$$

The moduli spaces $\mathcal{M}_{P}(x, y), \mathcal{M}_{O}$ embed, respectively, into certain Banach manifolds $\mathcal{B}_{P}(x, y), \mathcal{B}_{O}$. They and their reduced versions are thus endowed with the ambient topology, which we call $\mathcal{B}$-topology. The reduced moduli spaces $\hat{\mathcal{M}}_{P}, \hat{\mathcal{M}}_{O}$ are included in the following bigger spaces: the moduli space of broken trajectories, denoted $\hat{\mathcal{M}}_{P}^{+}$, and the moduli space of broken orbits, denoted $\hat{\mathcal{M}}_{O}^{+}$. The latter consists of the following more general objects:

A $\left(k\right.$-th) broken trajectory is an ordered set $\left\{\hat{u}_{0}, \hat{u}_{1}, \ldots, \hat{u}_{k}\right\}$, where $\hat{u}_{i}$ are reduced connecting flow lines, with the end point of $\hat{u}_{i}$ identified with the starting point of $\hat{u}_{i+1} \forall i \in\{0, \ldots, k-1\}$. These critical points are said to be the connecting rest points of the broken trajectory. The starting point of the broken trajectory is defined to be the starting point of $\hat{u}_{0}$, while its end point is defined to be the end point of $\hat{u}_{k}$. With the notion of starting and end points of a broken trajectory clarified, the space $\hat{\mathcal{M}}_{P}^{+}(x, y)$ now makes sense. The connecting flow line $\hat{u}_{i}$ are said to be the $i$-th components of the broken trajectory. In particular, any connecting flow line is a 0-th broken trajectory.

Similarly, when $k \in \mathbb{Z}^{+}$, a $k$-th broken orbit is a cyclically ordered set $\left\{\hat{u}_{1}, \hat{u}_{2}, \ldots, \hat{u}_{k}\right\}$, where $\hat{u}_{i}$ are reduced connecting flow lines, with the end point of $\hat{u}_{i}$ identified with the starting point of $\hat{u}_{i+1} \forall i \in \mathbb{Z} / k \mathbb{Z}$; a 0 -th broken orbit is by definition a closed orbit. Given representatives $u_{i}$ of the components $\hat{u}_{i}$, the homology class of the $k$-th broken orbit above is defined to be the homology class of the 1-cycle $\sum_{i=1}^{k} u_{i}$ in $\mathcal{C}$. With this explained, the definition of $\hat{\mathcal{M}}_{O}(A)$ easily extends to give $\hat{\mathcal{M}}_{O}^{+}(A)$.

The spaces of broken trajectories/orbits are endowed with the chain topology. They are stratified spaces, with the strata indexed by the set of rest points (regarded as an ordered set for broken trajectories, and as an cyclically ordered set for broken orbits). A stratum indexed by $S_{1}$ is in the closure of the stratum indexed by $S_{2}$ iff $S_{2} \subset S_{1}$ as ordered or cyclically ordered subsets.

The notion of grading and energy extend naturally to the moduli spaces of broken trajectories or broken orbits. Thus, we also have $\hat{\mathcal{M}}_{P}^{k,+}(\chi), \hat{\mathcal{M}}_{P}^{+}(\chi)^{\Re}$, 
$\hat{\mathcal{M}}_{P}^{k,+}(\chi)^{\Re}, \hat{\mathcal{M}}_{O}^{k,+}(\chi)^{\Re}$, etc., - moduli spaces of broken trajectories or orbits with the indicated grading and/or energy bound.

Generically, $\hat{\mathcal{M}}_{P}^{+}(x, y)$ or $\hat{\mathcal{M}}_{O}^{+}$are expected to be manifolds with corners. As the construction of $I_{\mathrm{F}}$ involves only moduli spaces of expected dimension not more than 1, we limit ourselves to the following special case:

Definition (lmb). Let $\hat{\mathcal{M}}^{+}$be a stratified space consisting of smooth strata of dimension no more than 1 , and $S \subset \hat{\mathcal{M}}^{+}$is a stratum of dimension $0 . \hat{\mathcal{M}}^{+}$ said to be locally a 1-manifold with boundary along $S$ (abbreviated $l m b$ ), if there is a homeomorphism $S \times[0,1)$ to a neighborhood of $S \subset \hat{\mathcal{M}}^{+}$, which restricts to a diffeomorphism over $S \times(0,1)$.

2.1.3. Floer systems. For generic parameter $\chi$, the moduli spaces are expected to satisfy the following regularity and compactness properties:

(FS1) (Structure of $\mathcal{P}_{\chi}$ ) $\mathcal{P}_{\chi}$ consists of finitely many non-degenerate points.

(FS2) (Structure of $\mathcal{M}_{P}(\chi)$ ) For any integer $k \leq 2$ and any $x, y \in$ $\mathcal{P}_{\chi}, \mathcal{M}_{P}^{k}(x, y ; \chi)$ is a (Zariski) smooth manifold of dimension $k$. Furthermore, for any real constant $\Re, \hat{\mathcal{M}}_{P}^{0}(\chi)^{\Re}$ consists of finitely many (Zariski) smooth points, $\hat{\mathcal{M}}_{P}^{1,+}(\chi)^{\Re}$ is compact, and is $\mathrm{lmb}$ along the strata of 1-th broken trajectories.

(FS3) (Structure of $\mathcal{M}_{O}(\chi)$ ) For any $k \leq 1$, the space $\mathcal{M}_{O}^{k}$ is a (Zariski) smooth manifold of dimension $k$. Moreover, for any real $\Re$, $\hat{\mathcal{M}}_{O}^{0}(\chi)^{\Re}$ consists of finitely many elements.

(FS4) (Orientation) The moduli spaces $\mathcal{M}_{P}^{k}(x, y), \mathcal{M}_{O}^{k}$ are orientable; and hence so are their reduced versions. Furthermore, the orientations of $\left\{\mathcal{M}_{P}^{k}(x, y)\right\}_{k \in \mathbb{Z} ; x, y \in \mathcal{P}}$ can be chosen coherently, and the orientation of $\mathcal{M}_{O}^{1}$ can be chosen to be grading-compatible. With respect to this choice,

$$
\partial \hat{\mathcal{M}}_{P}^{1,+}(x, y)=\coprod_{z \in \mathcal{P}} \hat{\mathcal{M}}_{P}^{0}(x, z) \times \hat{\mathcal{M}}_{P}^{0}(z, y) \text {. }
$$

The precise definition of "coherent orientation" and "grading-compatible orientation" will be postponed to II.7.2. Roughly speaking, a coherent orientation is a choice of orientations consistent with gluing, and a gradingcompatible orientation is one which allows a spectral-flow interpretation compatible with the spectral-flow interpretation of the absolute $\mathbb{Z} / 2$-grading ind. Note also that the definition of orientability here means the orientability of the relevant determinant line bundle; hence, the moduli space might be orientable even if it is not a manifold (see II.7 for details).

Remark. The issue of orientation is simpler in the context of Morse theory, when $\mathcal{C}$ is a finite-dimensional oriented manifold. In this case, the orientation 
of $\mathcal{C}$ gives a standard choice of the orientations of $\mathcal{M}_{P}$ and $\mathcal{M}_{O}$, via their interpretation as spaces of intersections (see e.g., [22]). As emphasized in Section 1.2.2, item 2, this interpretation is unavailable in Floer theory.

The formal flow associated to $\mathcal{V}_{\chi}$ is said to be a Floer system if (FS1-FS4) hold. An oriented Floer system is a Floer system together with a choice of coherent orientation on $\mathcal{M}_{P}$, a grading-compatible orientation on $\mathcal{M}_{O}^{1}$, and the induced orientation on the reduced moduli spaces.

2.2. Constructing $I_{\mathrm{F}}$ : the algebraic framework. Below is a straightforward adaptation of the construction of $[\mathbf{2 3}]$ to the Floer-theoretic settings.

2.2.1. The Novikov ring. Let $G$ be an abelian group, $R$ a ring, and $N: G \rightarrow \mathbb{R}$ a homomorphism. The Novikov ring $\operatorname{Nov}(G, N ; R)$ is the set of formal sums $\sum_{g \in G} a_{g} \cdot g$, with $a_{g} \in R$, such that for every $C \in \mathbb{R}$, the set $\left\{g \in G \mid N(g)<C\right.$ and $\left.a_{g} \neq 0\right\}$ is finite. $\operatorname{Nov}(G, N ; R)$ is a ring with the obvious addition and the convolution product (see e.g., $[\mathbf{2 0}]$ ).

Notice that $\operatorname{Nov}(G, 0, R)=R[G]$, and there is an inclusion

$$
i_{N}: R[G] \hookrightarrow \operatorname{Nov}(G, N ; R) .
$$

The Novikov ring should thus be viewed as a completion of the group ring.

The degree of $a$, denoted $\operatorname{deg}(a)$, is defined to be the minimum of $N(g)$ among $g$ such that $a_{g} \neq 0$. (Such minimum exists by the definition of Novikov rings.)

The notion of limit is defined for Novikov rings: $\lim _{n \rightarrow \infty} a_{n}=a$ for a sequence $\left\{a_{n} \in \operatorname{Nov}(G, N ; R)\right\}$ if $\forall \Re \in \mathbb{R}, \exists \Lambda(\Re)$ such that $\operatorname{deg}\left(a_{n}-a\right) \geq \Re$ $\forall n \geq \Lambda(\Re)$.

Given $a=\sum_{g} a_{g} g \in \operatorname{Nov}(G, N ; R)$, the "leading term" of $a$ is defined to be

$$
\operatorname{lt}(a):=\sum_{N(g)=\operatorname{deg}(a)} a_{g} g .
$$

$a-\operatorname{lt}(a)$ is called "higher order terms". Notice that lt defines a homomorphism

$$
\operatorname{Nov}(G, N ; R) /( \pm G) \rightarrow R[\operatorname{ker} N] /( \pm \operatorname{ker} N) .
$$

In this article,

$$
G \text { is a finitely generated abelian group; } R=\mathbb{Z} \text { or } \mathbb{Q} \text {. }
$$

In this case, the Novikov ring is commutative.

We shall often need to consider the rings of fractions of Novikov rings.

First, observe that a splitting

$$
G=\operatorname{ker} N \oplus G / \operatorname{ker} N,
$$

induces an embedding:

$$
Q(\operatorname{Nov}(G, N ; R)) \hookrightarrow \operatorname{Nov}(G / \operatorname{ker} N, N ; Q(R[\operatorname{ker} N])),
$$


and different embeddings are related by the natural action of the space of splittings ker $N$ on the right hand side.

Furthermore, in the case of (2), both sides of (3) are finite sums of fields. (As a special case, $Q(R[G])$ is a finite sum of fields, see e.g., [45] Section 3.1, [21] Lemma A.4). The embedding (3) is compatible with the decompositions on both sides as sums of fields.

Remark. In comparison with the first ring, the second ring in (3) has nicer properties (e.g., existence of the notions of degree, order, and limit), which the invariance proofs in $[\mathbf{2 1}, \mathbf{2 3}]$ made use of. There is a confusion between the two rings in $[\mathbf{2 3}]$, which also propagate to later papers. In these papers, the notion of order for elements in $Q(\operatorname{Nov}(G, N, R))$ should be understood in terms of the larger ring above through the embedding (3).

Through the embedding (3), we may extend the notion of leading term to:

$$
\text { lt : } Q(\operatorname{Nov}(G, N ; R)) / \pm G \rightarrow Q(R[\operatorname{ker} N]) / \pm \operatorname{ker} N \text {. }
$$

Note that the above map is independent of the choice of splitting, since we mod out ker $N$. The embedding $i_{N}$ also extends to the ring of fractions,

$$
i_{N}: Q(R[G]) \hookrightarrow Q(\operatorname{Nov}(G, N ; R)) .
$$

Later, we shall also use the same notation $i_{N}$ to denote the induced map from $Q(R[G]) /( \pm G)$ to $Q(\operatorname{Nov}(G, N ; R)) /( \pm G)$.

Let $\tilde{\mathbb{Q}}$ be a ring extension of $\mathbb{Q}$, for example, $\tilde{\mathbb{Q}}=\mathbb{Q}$ or the ring $Q(R[\operatorname{ker} N])$ in $(3) .{\text { Let } \operatorname{Nov}^{+}}^{+}(G, N ; \tilde{\mathbb{Q}}) \subset \operatorname{Nov}(G, N ; \tilde{\mathbb{Q}})$ denote the subset of elements of positive degree.

Let $\operatorname{Nov}^{1}(G, N ; \tilde{\mathbb{Q}}) \subset \operatorname{Nov}(G, N ; \tilde{\mathbb{Q}})$ be the subgroup consisting of elements of the form $1+c, c \in \operatorname{Nov}^{+}(G, N ; \tilde{\mathbb{Q}})$. The exponential

$$
\exp : \operatorname{Nov}^{+}(G, N ; \tilde{\mathbb{Q}}) \rightarrow \operatorname{Nov}^{1}(G, N ; \tilde{\mathbb{Q}}) \hookrightarrow Q(\operatorname{Nov}(G, N ; \tilde{\mathbb{Q}}))
$$

is well-defined via the usual power series. Conversely, the logarithm

$$
\ln : \operatorname{Nov}^{1}(G, N ; \tilde{\mathbb{Q}}) \rightarrow \operatorname{Nov}^{+}(G, N ; \tilde{\mathbb{Q}})
$$

also makes sense formally.

Novikov rings arise naturally in Morse-Novikov theory as the coefficient rings.

2.2.2. The Floer-Novikov complex. Each oriented Floer system (e, $\mathfrak{H}$, ind; $\left.y_{\chi}, \mathcal{V}_{\chi}\right)$ is associated with a (twisted) Floer complex as follows.

Let the chain groups $\tilde{C F}$ be the free $\operatorname{Nov}\left(\mathfrak{H},-\left\langle y_{\chi}\right\rangle ; \mathbb{Z}\right)$-module generated by elements in $\tilde{\mathcal{P}}_{\chi}$, on which $\mathfrak{H}$ acts by deck-transformation.

Let the boundary map $\tilde{\partial}_{\mathrm{F}}: \tilde{C F} \rightarrow \tilde{\mathrm{CF}}$ be defined by

$$
\tilde{\partial}_{\mathrm{F}}(x,[w])=\sum_{(y,[v]) \in \tilde{\mathcal{P}}} \chi\left(\hat{\mathcal{M}}_{P}^{0}((x,[w]),(y,[v]))\right)(y,[v]) .
$$


By (FS2), $\tilde{\partial}_{\mathrm{F}}$ is a well-defined $\operatorname{Nov}\left(\mathfrak{H},-\left\langle y_{\chi}\right\rangle ; \mathbb{Z}\right)$-linear transformation, and $\tilde{\partial}_{\mathrm{F}}^{2}=0$.

Via the relative $\mathbb{Z}$-grading on $\tilde{\mathcal{P}}_{\chi}, \tilde{\mathrm{CF}}$ splits as:

$$
\left(\tilde{\mathrm{CF}}, \tilde{\partial}_{\mathrm{F}}\right)=\bigoplus_{k}\left(\tilde{\mathrm{CF}}_{k}, \tilde{\partial}_{\mathrm{F}, k}\right)
$$

where $k$ is in an affine space under $\mathbb{Z}$. Each summand $\tilde{C F}_{k}$ is a free $\Lambda_{\mathrm{F}}$-module of finite rank, where

$$
\Lambda_{\mathrm{F}}:=\operatorname{Nov}\left(\operatorname{ker} \psi,-\left\langle y_{\chi}\right\rangle ; \mathbb{Z}\right) \subset \operatorname{Nov}\left(\mathfrak{H},-\left\langle y_{\chi}\right\rangle ; \mathbb{Z}\right) .
$$

Furthermore, they satisfy the periodicity condition

$$
\left(\tilde{\mathrm{CF}}_{k}, \tilde{\partial}_{\mathrm{F}, k}\right)=\left(\tilde{\mathrm{CF}}_{k+2 \mathbb{N}_{\psi}}, \tilde{\partial}_{\mathrm{F}, k+2 \mathbb{N}_{\psi}}\right) \text {, }
$$

$2 \mathbb{N}_{\psi}$ being the ged of the values of $\psi$.

As the relative $\mathbb{Z}$-valued index on $\tilde{\mathcal{P}}_{\chi}$ reduces to an absolute $\mathbb{Z} / 2 \mathbb{Z}$-valued grading ind, there is also a reduced version of Floer complex,

$$
\left(\mathrm{CF}, \partial_{\mathrm{F}}\right)=\bigoplus_{i}\left(\mathrm{CF}_{i}, \partial_{\mathrm{F}, i}\right),
$$

where $i$ takes values in the group $\mathbb{Z} / 2 \mathbb{Z}$, and $\mathrm{CF}_{i}$ is the free $\Lambda_{\mathrm{F}}$-module generated by all elements in $\mathcal{P}_{\chi}$ of index $i$.

2.2.3. The Reidemeister torsion. We now specify the version of torsion used in this paper.

(1) Suppose first for simplicity that the coefficient $\operatorname{ring} F$ is a field. Let $\left(C_{i}, \partial_{i}\right), i \in \mathbb{Z} / 2 \mathbb{Z}$ be a complex of finite-dimensional $F$-vector spaces, and $Z_{i}, B_{i}$ be, respectively, the subspace of cycles and boundaries in $C_{i}$. The standard short exact sequences $0 \rightarrow Z_{i} \rightarrow C_{i} \rightarrow B_{i-1} \rightarrow 0$ and $0 \rightarrow B_{i} \rightarrow Z_{i} \rightarrow H_{i} \rightarrow 0$ induce a canonical isomorphism

$$
\mathcal{T}: \bigotimes_{i} \operatorname{det}\left(C_{i}\right)^{(-1)^{i}} \longrightarrow \bigotimes_{i} \operatorname{det}\left(H_{i}\right)^{(-1)^{i}} \text {. }
$$

Let $e$ be an ordered basis for $C_{*}$, that is, an ordered basis $e_{i}$ for each $C_{i}$. Let $h$ be an ordered basis for $H_{*}$. Let $[e] \in \bigotimes_{i} \operatorname{det}\left(C_{i}\right)^{(-1)^{i}}$ and $[h] \in \bigotimes_{i} \operatorname{det}\left(H_{i}\right)^{(-1)^{i}}$ denote the resulting volume forms.

In this simplest case, when the coefficient ring is a field, define the Reidemeister torsion

$$
\tau\left(C_{*} ; e\right):=\left\{\begin{array}{cl}
\mathcal{T}([e]) /[h] \in F^{\times} & \text {if } H_{*}=0 \\
0 & \text { otherwise }
\end{array}\right.
$$

(2) For our applications, the coefficient ring of the complex is a Novikov ring of the type specified in (2) (including group rings). We saw that in this case it is in general not a field, but its total ring of fractions is a direct sum of fields. 
Definition. [45] Let $R$ be a ring, and assume that its total ring of fractions $Q(R)$ is a finite sum of fields, $Q(R)=\bigoplus_{j} F_{j}$. Let $\left(C_{i}, \partial_{i}\right), i \in \mathbb{Z} / 2 \mathbb{Z}$ be a complex of finitely generated free $R$-modules with an ordered basis $e$. Then

$$
\tau\left(C_{*}, e\right):=\sum_{j} \tau\left(C_{*} \otimes_{R} F_{j}, e \otimes 1\right) \in \bigoplus_{j} F_{j}=Q(R) .
$$

(3) Associated to an oriented Floer system, the Reidemeister torsion of the Floer complex is defined as

$$
\tau_{\mathrm{F}}:=\tau\left(\mathrm{CF}, e_{\mathcal{P}}\right) \in Q\left(\Lambda_{\mathrm{F}}\right) /( \pm \operatorname{ker} \psi)
$$

where $e_{\mathcal{P}}$ is an ordered basis of $\mathrm{CF}$ given by an ordering of elements in $\mathcal{P}$, and a lift Lf $: \mathcal{P} \rightarrow \tilde{\mathcal{P}}$. In the case when $\mathbb{N}_{\psi} \neq 0$, we require further:

- that the lift Lf is such that $|\operatorname{gr}(\operatorname{Lf}(x), \operatorname{Lf}(y))|<2 \mathbb{N}_{\psi} \forall x, y \in \mathcal{P}$, and

- a choice of an $A_{0} \in \mathfrak{H}$, such that $\psi\left(A_{0}\right)=2 \mathbb{N}_{\psi}$.

The set of all $\left(n A_{0}\right) \cdot \operatorname{Lf}(x), n \in \mathbb{Z}, x \in \mathcal{P}$ then forms a basis of $\tilde{C F}$ as a graded free $\Lambda_{\mathrm{F}}$-module.

Different ordering of the critical points results in a possible change of sign for $\tau\left(\mathrm{CF}, e_{\mathcal{P}}\right)$, and different such lifts and $A_{0}$ result in a multiplication of $\tau$ by an element in ker $\psi$; so by modding out \pm ker $\psi$ in the definition we obtain an invariant independent of these choices.

(4) Another version of torsion that is important in topology is the Reidemeister torsion of a manifold, denoted $\tau(M)$. Let $M$ be a manifold with a cell-decomposition, such that the cell chain complex $C_{*}(M)$ is a finite complex of finite-rank $\mathbb{Z}$-modules. The universal abelian covering $\tilde{M}$ is endowed with an induced equivariant cell-decomposition, and $C_{*}(\tilde{M})$ is a $\mathbb{Z}\left[H_{1}(M ; \mathbb{Z})\right]$-module.

$$
\tau(M):=\tau\left(C(\tilde{M}), e_{M}\right) \in Q\left(\mathbb{Z}\left[H_{1}(M ; \mathbb{Z})\right]\right) / \pm H_{1}(M ; \mathbb{Z}),
$$

where $e_{M}$ is an ordered basis consisting of lifts of cells in $M$.

2.2.4. The zeta function and the counting invariant $I_{\mathrm{F}}$. Fix an oriented Floer system $\left(\mathcal{C}, \mathfrak{H}\right.$, ind; $\left.\mathfrak{y}_{\chi}, \mathcal{V}_{\chi}\right)$.

To count the closed orbits, we imitate the definition of the dynamical zeta function, and define the the Floer-theoretic zeta function as 


$$
\begin{aligned}
\zeta_{\mathrm{F}} & :=\exp \left(\sum_{A \in \operatorname{ker} \psi, A \neq 0} \chi\left(\hat{\mathcal{M}}_{O}(A)\right) A\right) \\
= & \exp \left(\sum_{A \in \operatorname{ker} \psi, A \neq 0} \sum_{u \in \hat{\mathcal{M}}_{O}(A)} \frac{\operatorname{sign}(u)}{\operatorname{mult}(u)} A\right) \\
& \in \operatorname{Nov}^{1}\left(\operatorname{ker} \psi,-\left\langle y_{\chi}\right\rangle ; \mathbb{Q}\right) \subset Q\left(\operatorname{Nov}\left(\operatorname{ker} \psi,-\left\langle y_{\chi}\right\rangle ; \mathbb{Q}\right)\right) .
\end{aligned}
$$

In (5), $\chi$ is the "orbifold Euler number", and we shall denote the exponent therein by $\eta_{\mathrm{F}}$. By $(\mathrm{FS} 3), \eta_{\mathrm{F}} \in \operatorname{Nov}^{+}\left(\operatorname{ker} \psi,-\left\langle y_{\chi}\right\rangle ; \mathbb{Q}\right)$, and the exponential is well-defined.

Finally, viewing both $\zeta_{\mathrm{F}}$ and $\tau_{\mathrm{F}}$ as elements in $Q\left(\operatorname{Nov}\left(\operatorname{ker} \psi,-\left\langle y_{\chi}\right\rangle ; \mathbb{Q}\right)\right) / \pm$ ker $\psi, I_{\mathrm{F}}$ is simply defined as the product

$$
I_{\mathrm{F}}:=\zeta_{\mathrm{F}} \tau_{\mathrm{F}} \in Q\left(\operatorname{Nov}\left(\operatorname{ker} \psi,-\left\langle y_{\chi}\right\rangle ; \mathbb{Q}\right)\right) / \pm \operatorname{ker} \psi .
$$

Remark. Ideally, $I_{\mathrm{F}}$ should be defined in $Q\left(\Lambda_{\mathrm{F}}\right) / \pm \operatorname{ker} \psi$ instead of the above larger monoid. However, this would require proving a product formula similar to $[\mathbf{2 3}]$ equation $(2)$ to ensure that $\zeta_{\mathrm{F}} \in \operatorname{Nov}^{1}\left(\operatorname{ker} \psi,-\left\langle y_{\chi}\right\rangle ; \mathbb{Z}\right)$.

2.3. Statement of the main results. Let $(M, \omega)$ be a closed, connected symplectic manifold of dimension $2 n$, and let $f: M \rightarrow M$ be a smooth, (possibly non-Hamiltonian) symplectomorphism.

Let $c_{1}:=c_{1}(T M)$. We shall need the notion of weak monotonicity and a stronger companion version.

2.3.1. Definition. A symplectic manifold $M$ is weakly monotone if $\omega(A)>$ 0 for all $A \in H_{2}(M)$ in the image of $\pi_{2}(M)$ under the Hurewicz map satisfying $0<c_{1}(T M)(A)<n-2$.

It is said to be $w^{+}$-monotone if $\omega(A)>0$ for all $A \in H_{2}(M)$ in the image of $\pi_{2}(M)$ under the Hurewicz map satisfying $0<c_{1}(T M)(A) \leq n-2$.

It is clear from the definition that monotonicity implies $w^{+}$-monotonicity, which in turn implies weak-monotonicity. Examples of $w^{+}$-monotone symplectic manifolds include Fano and Calabi-Yau manifolds, and any symplectic manifold of dimension less than six.

Our main result concerns a " $w^{+}$-monotone" symplectic manifold. The assumption of $w^{+}$-monotonicity is mainly here for simplicity: In view of $[\mathbf{1 7}, \mathbf{3 3}, \mathbf{3 8}]$, we expect the result to hold for general symplectic manifolds. In fact, most of the proofs contained in this article work for weakly monotone manifolds. The stronger $w^{+}$-monotonicity assumption is imposed in Section 6 to shorten the discussion on the structure of parameterized moduli spaces. 
It is convenient to introduce the mapping torus of $f$ :

$$
T_{f}:=\{(x, t): x \in M, t \in[0,1]\} /(x, 0) \sim(f(x), 1),
$$

which fibers over the circle of unit length $S_{1}^{1}$ :

$$
M \stackrel{\iota_{f}}{\longrightarrow} T_{f} \stackrel{\pi_{S}}{\longrightarrow} S_{1}^{1} .
$$

Notation. By restricting to the fibers of $\pi_{S}$, a function $S$ on $T_{f}$ or a section of a bundle over $T_{f}$ corresponds to a path of functions/sections over $M$ with matching conditions on the two ends determined by $f$. We shall denote these functions/sections over $M$ by $S_{t}, t \in[0,1]$.

2.3.2. Definition. The $f$-twisted loop space $\mathcal{L}_{f} M$ (usually just called the "loop space") is the space of $L_{1}^{2}$ sections of $T_{f}$.

Given $\gamma_{0} \in \mathcal{L}_{f} M_{\gamma_{0}}$, let $\mathcal{L}_{f} M_{\gamma_{0}}=$ : $\mathcal{C}$ be the path component containing $\gamma_{0}$.

There is a natural map

$$
\operatorname{im}: \pi_{1}(\mathcal{C}) \rightarrow H_{2}\left(T_{f} ; \mathbb{Z}\right)
$$

sending each 1-cycle $\gamma: S^{1} \rightarrow \mathcal{L}_{f} M_{\gamma_{0}}$ representing a homotopy class, to the corresponding map from $S^{1} \times S^{1}$ to $T_{f}$.

Let $K$ be the subbundle of $T T_{f}$ consisting of tangent vectors to the fibers.

A complex structure $J$ of the bundle $K$ is said to be $\omega$-compatible if $J_{t}$ are $\omega$-compatible for all $t \in[0,1]$. Namely, $\omega\left(J_{t} \cdot, \cdot\right)$ gives a Riemannian metric on $M^{1}$. Let $\partial_{K}$ denote the Banach manifold of $C_{\epsilon} \omega$-compatible complex structures on $K$.

Let $X=\left\{X \mid X \in C_{\epsilon}\left(T_{f}, K\right), X_{t}\right.$ is a symplectic vector field $\left.\forall t\right\}$.

Let $\mathcal{H}=C_{\epsilon}\left(T_{f}\right)$. Given $H \in \mathcal{H}$, let $\chi_{H} \in \mathcal{X}$ be defined by

$$
\left(\chi_{H}\right)_{t}=\chi_{H_{t}} ; \quad \omega\left(\chi_{H_{t}}, \cdot\right)=d H_{t} .
$$

We shall describe in Section 3 , a Floer theory $\left(\mathcal{C}, \mathfrak{H}\right.$, ind; $\left.\mathcal{Y}_{\chi}, \mathcal{V}_{\chi}\right)$ satisfying the framework outlined in Section 2.1, in which $\mathcal{C}=\mathcal{L}_{f} M_{\gamma_{0}}, \mathfrak{H}=$ Image $(\mathrm{im}) \subset H_{2}\left(T_{f}\right)$, ind is a variant of the Conley-Zehnder index, and $y_{\chi}, \nu_{\chi}$ are parameterized by $\chi=(J, X) \in \partial_{K} \times X$. For certain generic $(J, X)$ which we call "regular pairs", the associated formal flow is an oriented Floer system (see Section 3 and II.7.2). Thus, for each regular pair $(J, X)$ the construction of Section 2.2 defines an $I_{\mathrm{F}}$, which we denote by $I_{\mathrm{F}}^{f,\left[\gamma_{0}\right]}(M ; J, X)$.

For fixed $f,\left[\gamma_{0}\right]$, the cohomology class $\left[y_{\chi}\right]$ only depends on the flux of $X$. In particular, $I_{\mathrm{F}}^{f,\left[\gamma_{0}\right]}(M ; J, X)$ and $I_{\mathrm{F}}^{f,\left[\gamma_{0}\right]}\left(M ; J^{\prime}, X^{\prime}\right)$ take values in the same monoid if $X-X^{\prime}$ is Hamiltonian. Our main result concerns the invariance properties of $I_{\mathrm{F}}^{f,\left[\gamma_{0}\right]}(M ; J, X)$.

\footnotetext{
${ }^{1}$ This definition differs by a sign from some literature, and is convenient for constructing Floer homology instead of Floer cohomology.
} 


\subsubsection{Theorem.}

(a) Let $(M, \omega)$ be a $w^{+}$-monotone symplectic manifold, and let $f$ be a symplectomorphism of $M$ and $\gamma_{0} \in \mathcal{L}_{f} M$ be chosen as earlier. Suppose $\left(J_{1}, X+\chi_{H_{1}}\right),\left(J_{2}, X+\chi_{H_{2}}\right) \in \mathcal{J}_{K} \times \mathcal{X}$ are two regular pairs. Then

$$
I_{\mathrm{F}}^{f,\left[\gamma_{0}\right]}\left(M ; J_{1}, X+\chi_{H_{1}}\right)=I_{\mathrm{F}}^{f,\left[\gamma_{0}\right]}\left(M ; J_{2}, X+\chi_{H_{2}}\right) .
$$

(b) Suppose $(M, \omega)$ is monotone. Then there exists an

$$
I_{\mathrm{F}}^{f,\left[\gamma_{0}\right]}(M) \in Q(\mathbb{Z}[\operatorname{ker} \psi]) / \pm \operatorname{ker} \psi \quad \text { such that }
$$

$$
I_{\mathrm{F}}^{f,\left[\gamma_{0}\right]}(M ; J, X)=i_{-\left[y_{X}\right]} I_{\mathrm{F}}^{f,\left[\gamma_{0}\right]}(M) \quad \text { for any regular pair }(J, X) .
$$

We shall review in Section 3.1.4 a well-known principle that relates varying $f,\left[\gamma_{0}\right]$ by symplectic isotopies and varying the symplectic vector field $X$. According to this principle, the earlier theorem implies that $I_{\mathrm{F}}$ is invariant under Hamiltonian or symplectic isotopies of $f,\left[\gamma_{0}\right]$, in the case of (a) or (b), respectively. In particular, under the assumptions of Theorem 2.3.3 (b), this implies that $I_{\mathrm{F}}$ depends only on the symplectic mapping class of $f$ and the conjugacy class of $\left[\gamma_{0}\right] \in \pi_{0}\left(\mathcal{L}_{f} M\right)$ under the standard action of $\pi_{1}\left(\operatorname{Symp}_{0}(M)\right)$ on $\pi_{0}\left(\mathcal{L}_{f} M\right)$. $\left(\operatorname{Symp}_{0}(M)\right.$ denotes the path component of the symplectomorphism group of $M$ containing the identity.)

Due to this principle, we shall say that $I_{\mathrm{F}}$ is invariant under Hamiltonian isotopies when (7) holds; similarly, we say that $I_{\mathrm{F}}$ is invariant under symplectic isotopies when (8) holds.

Combining this result with the main theorem in $[\mathbf{2 1}, \mathbf{2 3}]$, we can compute $I_{\mathrm{F}}^{f,\left[\gamma_{0}\right]}(M)$ in the following basic case:

2.3.4. Corollary. Suppose $M$ is monotone, and $f$ is connected to the identity via the symplectic-isotopy $f_{t}, t \in[0,1]$, with $f_{0}=\mathrm{Id}, f_{1}=f$. Let $\gamma_{0}$ be the path $\gamma_{0}(t)=f_{t}\left(p_{0}\right)$ for a base point $p_{0} \in M$. In this case $\operatorname{ker} \psi=\left.H_{1}(M ; \mathbb{Z}) \oplus \operatorname{ker} c_{1}(T M)\right|_{\pi_{2}(M)} ;$ let $\iota: H_{1}(M ; \mathbb{Z}) \rightarrow \operatorname{ker} \psi$ denote the inclusion, and let $\iota_{*}: Q\left(\mathbb{Z}\left[H_{1}(M ; \mathbb{Z}]\right) / \pm H_{1}(M ; \mathbb{Z}) \rightarrow Q(\mathbb{Z}[\operatorname{ker} \psi]) / \pm \operatorname{ker} \psi\right.$ denote the induced map. Then

$$
I_{\mathrm{F}}^{f,\left[\gamma_{0}\right]}(M)=\iota_{*} \tau(M) .
$$

In Section 4, we shall state a more general invariance theorem and explain how Theorem 2.3.3 follows as a consequence.

2.3.5. Some immediate application. Since critical points in the Floer theory correspond to symplectic fixed points, non-triviality of the Reidemeister torsion $\tau_{\mathrm{F}}$ will guarantee the existence of symplectic fixed points. Here is a sample result in this direction: 
Corollary. Let $M, f, \gamma_{0}$ be as in Corollary 2.3.4. In addition, suppose that lt $\tau(M) \neq 1$. Then $f$ has a fixed point.

This is a direct consequence of the computation in Corollary 2.3.4 and the observation that $\operatorname{lt}\left(\zeta_{\mathrm{F}}\right)=1$ by definition.

More applications shall be discussed in subsequential articles (e.g., [2931]), including applications exploiting higher order terms of $I_{\mathrm{F}}$. Here, we shall content us with the following few remarks.

\section{Remarks.}

(a) The abelian version of torsion introduced in this paper is not amenable for obtaining quantitative results (e.g., getting fixed-point-number bounds). A possible refinement is to work with the non-abelian Whitehead torsion instead: one would then obtain a lower bound on fixed-point-numbers in terms of the minimal rank of representations of the relevant Whitehead torsion. This is, however, often very difficult to compute, and thus does not seem an effective way for obtaining such results. Some results in this direction for the Floer theory of Lagrangian intersections (i.e., Example 1.1.3) may be found in [9].

(b) The Morse-theoretic picture leads one to expect a converse of such existence theorems: that the critical points of the Floer theory (i.e., symplectic fixed points or Lagrangian intersection points) can be removed via a Hamiltonian isotopy when both the Floer homology and the Floer-theoretic Whitehead torsion vanish. This is the outstanding "symplectic s-cobordism conjecture" posed by Fukaya [15].

(c) The requirement that $M$ is both closed and symplectic places a very strong constraint on the topology of $M$, and it is not easy to find closed symplectic manifolds with interesting Reidemeister torsion (i.e., the field components of $\tau(M)$ are not all 0 or 1 ). We shall show in the sequels to this paper that interesting examples are easier to produce in other versions of Floer theories, for example, those in Examples 1.1.1-1.1.3. See also an $S^{1}$-equivariant version in [30]. For instance, one may take $M$ to be a Stein manifold in Example 1.1.1. By the combinatorial description of Stein manifolds ([8]), $M$ has very flexible topology and it is easy to produce $M$ with interesting torsion accordingly.

\section{Floer theory of symplectomorphisms}

Via a simple modification of Floer's work, the Floer homology of a general symplectomorphism was first introduced by Dostoglou and Salamon in [6] for monotone symplectic manifolds. The virtual moduli method being available today, it should be defined for general symplectic manifolds. In this paper, we however, take the middle ground by imposing the weakly monotonicity condition, to avoid the virtual moduli method. 
Though it is more-or-less standard, there does not seem to be a good reference that deals directly with the situation we need. We shall therefore, go into some details on the construction of this version of Floer theory, by indicating how the available literature should be modified.

3.1. Setup and basics. Recall the definitions and notation from Section 2.3 .

3.1.1. Topology of the loop space. Fix a base point $p_{0} \in M$, and let $\gamma_{0} \in \mathcal{C}$ be a path with $\gamma_{0}(0)=p_{0}$. We gather some basic facts about $\mathcal{L}_{f} M_{\gamma_{0}}=$ : $\mathcal{C}$ below.

First, notice that the fiber-bundle structure of $T_{f}$

$$
M \stackrel{\iota_{f}}{\longrightarrow} T_{f} \stackrel{\pi_{S}}{\longrightarrow} S_{1}^{1}
$$

gives rise to the following useful Mayer-Vietoris type sequence:

$$
\cdots H_{2}(M ; \mathbb{Z}) \stackrel{1-f_{*}}{\longrightarrow} H_{2}(M ; \mathbb{Z}) \stackrel{\iota_{f *}}{\longrightarrow} H_{2}\left(T_{f}\right) \stackrel{\partial_{f}}{\longrightarrow} H_{1}(M ; \mathbb{Z}) \stackrel{1-f_{*}}{\longrightarrow} \cdots
$$

Let $c_{1}^{f}:=c_{1}(K)$; since $f$ is a symplectomorphism, $\omega$ defines a two-form on $T_{f}$, which we denote by $\omega^{f}$. The restrictions of the cohomology classes $c_{1}^{f},\left[w^{f}\right]$ define two homomorphisms $\psi_{c}: \mathfrak{H} \rightarrow \mathbb{Z}, \psi_{\omega}: \mathfrak{H} \rightarrow \mathbb{R}$, respectively. They are related to the SF-homomorphism and the cohomology class $\left[y_{\chi}\right]$, respectively.

Next, note the fibration

$$
\Omega M \rightarrow \mathcal{L}_{f} M \stackrel{e_{f}}{\rightarrow} M,
$$

where $\Omega M$ is the loop space (with based point) of $M$, and $e_{f}(\gamma)=\gamma(0)$ is the end point map. Thus, we have the associated homotopy sequence:

$$
\cdots \pi_{2}(M ; p) \rightarrow \pi_{1}\left(\mathcal{L}_{f} M ; \gamma_{0}\right) \rightarrow \pi_{1}(M ; p) \stackrel{\delta_{f, \gamma_{0}}}{\longrightarrow} \pi_{1}(M ; p) \cdots
$$

The two exact sequences (9) and (11) fit into the following commutative diagram with im:

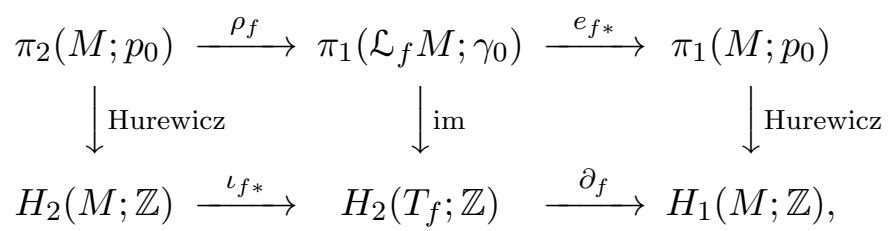

where "Hurewicz" denotes the Hurewicz map.

3.1.2. The space of almost complex structures. Given a $\omega$-compatible almost complex structure $J_{0}$ over $M$, let $\mathcal{S}\left(A, J_{0}\right)$ be the space of simple (i.e., non-constant and not multiply-covered) smooth $J_{0}$-holomorphic spheres with homology class $A$ in $M$. The automorphism group of $\mathbb{C} P^{1}$, 
$G:=\operatorname{PSL}(2, \mathbb{C})$, acts freely on $\mathcal{S}\left(A, J_{0}\right)$ when $A$ is non-trivial. Let $\mathcal{S}\left(J_{0}\right):=\coprod_{A} \mathcal{S}\left(A, J_{0}\right)$.

A $J \in \mathcal{J}_{K}$ is said to be regular if the space

$$
S(A, J) / G:=\bigcup_{t} S\left(A, J_{t}\right) / G
$$

is a smooth manifold of expected dimension (i.e., $2 n+2 c_{1}(A)-5$ ).

We denote by $\mathcal{J}_{K}^{\text {reg }} \subset \mathcal{J}_{K}$ the subset consisting of regular elements, and summarize some useful facts on such regular elements as follows.

Lemma. Let $M$ be weakly monotone.

(a) If $J \in \mathcal{J}_{K}^{\mathrm{reg}}$, then for all $t \in S^{1}, J_{t}$ is semi-positive in the sense that any $J_{t}$-holomorphic sphere has non-negative Chern number.

(b) The subset $\partial_{K}^{\mathrm{reg}} \subset \partial_{K}$ is Baire.

(c) Part (b) above may be refined as follows. Let $y: S^{1} \rightarrow T_{f}$ be a section, and let $U_{y}$ be a small tubular neighborhood of the image of $y$ in $T_{f}$. Let $\underline{J} \in \mathcal{J}_{K}$, and let

$$
\partial_{K}\left(\underline{J}, U_{y}\right):=\left\{J\left|J \in \mathcal{J}_{K}, J\right|_{U_{y}}=\left.\underline{J}\right|_{U_{y}}\right\},
$$

endowed with the obvious Banach manifold structure. Then the space of regular elements in $\mathcal{J}_{K}\left(\underline{J}, U_{y}\right)$ is Baire.

Proof. (a) follows from the proof of [34] Lemma 5.1.3. (b) is a result of a standard transversality argument (see e.g., [34] Theorem 3.1.3). To see (c), one simply augments the proof of [34] Theorem 3.1 .3 by the observation that if $U_{y}$ is small enough, every $J_{t}$-holomorphic sphere exits the small neighborhood of $y(t)$ obtained by restricting $U_{y}$ to the fiber over $t$. (See e.g., Remark 5.2 of [14] for an argument of the same type.)

Finally, it is useful to introduce the following notation:

Let $M_{c}\left(J_{t}\right) \subset M$ be the image under the evaluation map

$$
\text { ev : } \coprod_{A, c_{1}(A) \leq c} \mathcal{S}\left(J_{t}, A\right) \times_{G} S^{2} \rightarrow M, \quad \operatorname{ev}(u, \theta)=u(\theta) .
$$

Let $M_{c}(J) \subset T_{f}$ be the subspace such that its intersection with the fiber over $t$ is $M_{c}\left(J_{t}\right)$.

\subsubsection{The action 1-form and its associated formal flow.}

Notation. For $X \in \mathcal{X}$, let $\theta_{X, t}:=\iota\left(X_{t}\right) \omega . \theta_{X}:=\int \theta_{X, t} d t ;\left[\theta_{X}\right] \in H^{1}(M)$ is the flux of $X$. In addition, given $J \in \mathcal{J}_{K}$, let $\check{\theta}_{X, t}^{J_{t}}$ be the dual vector field of $\theta_{X, t}$ with respect to the metric associated to $J_{t}$, and let $\check{\theta}_{X}^{J}$ denote the section $\left\{\check{\theta}_{X, t}^{J_{t}}\right\}_{t \in S^{1}}$ of $K$. The superscript $J_{t}$ or $J$ will often be omitted when $J$ is fixed. 
Conversely, given a closed 1-form $\theta$ on $M, X_{\theta}$ denotes the symplectic vector field such that $\theta=\iota\left(X_{\theta}\right) \omega$.

In this article, $y_{\chi}$ and $\tilde{\mathcal{A}}_{\chi}$ will be the action 1 -form $y_{X}$ and action functional $\tilde{\mathcal{A}}_{X}$, given as follows.

For any $X \in X$ and $\xi \in T_{\gamma} \mathcal{C}=L_{1}^{2}\left(\gamma^{*} K\right)$,

$$
y_{X}(\gamma)(\xi):=-\int_{S^{1}} \omega\left(\partial_{t} \gamma(t), \xi(t)\right) d t+\int_{S^{1}} \theta_{X, t}(\xi(t)) d t
$$

Since, $H_{2}\left(T_{f} ; \mathbb{Z}\right)$ is abelian, the map im factors as a composition of the Hurewicz map and a homomorphism $\underline{\mathrm{im}}: H_{1}(\mathcal{C} ; \mathbb{Z}) \rightarrow H_{2}\left(T_{f} ; \mathbb{Z}\right)$. Similarly, we may define the homomorphism $\underline{e}_{f *}: H_{1}(\mathcal{C} ; \mathbb{Z}) \rightarrow H_{1}(M ; \mathbb{Z})$ so that $\underline{e}_{f *} \circ$ Hurewicz $=$ Hurewicz $\circ e_{f *}$. The cohomology class $\left[y_{X}\right] \in H^{1}(\mathcal{C})$ may be expressed in these homomorphisms as

$$
\left[y_{X}\right]=-\underline{\mathrm{im}}^{*}\left[\omega^{f}\right]+\underline{e}_{f}^{*}\left[\theta_{X}\right]=\underline{\mathrm{im}}^{*}\left(-\left[\omega^{f}\right]+\partial_{f}^{*}\left[\theta_{X}\right]\right),
$$

where $(12)$ was used for the second equality. We see that $\left[y_{X}\right]: H_{1}(\mathcal{C} ; \mathbb{Z}) \rightarrow$ $\mathbb{R}$ factors through $\underline{\mathrm{im}}$ and a $\left\langle y_{X}\right\rangle \in \operatorname{Hom}(\mathfrak{H}, \mathbb{R})$ :

$$
\left\langle y_{X}\right\rangle=-\psi_{\omega}+\partial_{f}^{*}\left[\theta_{f}\right] \text {. }
$$

Hence, $y_{X}$ lifts to an exact form on the $\mathfrak{H}$-cover $\tilde{\mathfrak{C}}$, meeting the requirement in Section 2.1.

On the other hand, given $J \in \mathcal{J}_{K}$, we have a Riemannian metric on $T_{f}$ by the $\omega$-compatibility of $J_{t}$, which in turn defines a metric for $\mathcal{L}_{f} M$.

The vector field dual to $-y_{X}$ with respect to this metric is $-\mathcal{V}_{X}$,

$$
\mathcal{V}_{X}(\gamma):=J_{t}(\gamma) \frac{\partial \gamma}{\partial t}+\check{\theta}_{X, t}^{J_{t}}(\gamma) \quad \text { for } \gamma \in \mathcal{C}
$$

Thus, a formal flow line of $\mathcal{V}_{X}$ is a solution to the perturbed CauchyRiemann equation

$$
\bar{\partial}_{J X} u:=\frac{\partial u}{\partial s}+J_{t}(u) \frac{\partial u}{\partial t}+\check{\theta}_{X, t}^{J_{t}}(u)=0,
$$

where $t \in S_{1}^{1}$, and $u$ is a smooth $M$-valued function on $(s, t)$ such that $u(s, \cdot) \in \mathcal{C} \forall s ; s \in \mathbb{R}$ when $u \in \mathcal{M}_{P}$, while $s \in S_{T}^{1}$ for some $T>0$ if $u \in \mathcal{M}_{O}$.

The energy of a solution $u$ to (15) is

$$
\mathcal{E}(u)=\int\left|\partial_{s} u\right|^{2} d s d t
$$

A straightforward computation confirms that this agrees with the definition for energy of closed orbits or connecting flow lines given in terms of $y_{\chi}$ and $\tilde{\mathcal{A}}_{\chi}$ in Section 2.1.

The various moduli spaces in this Floer theory depend on $\gamma_{0}, M, f$, and $J, X$. All these shall be incoporated in the full notation for the moduli spaces, for example, $\mathcal{M}_{P}^{\gamma_{0}}(M, f ; J, X)$. However, when $\gamma_{0}, M, f$ are fixed, 
they will often be omitted from the notation. So is the parameter $(J, X)$, if it is clear from the context.

We now introduce the chain topology on $\hat{\mathcal{M}}_{P}^{+}$and $\hat{\mathcal{M}}_{O}^{+}$. First, let $p_{2}: \mathbb{R} \times S^{1} \rightarrow S^{1}$ denote the projection to the second factor. For a point $a \in S^{1}$ and a representative $(T, u)$ of $(T, \hat{u}) \in \hat{\mathcal{M}}_{O}$, we define a $u_{a} \in L_{1, \mathrm{loc}}^{p}\left(\mathbb{R} \times S^{1} ; p_{2}^{*} T_{f}\right)$ so that:

- Over $[-T / 2, T / 2] \times S^{1}, u_{a}$ agrees with a representative of $(T, \hat{u})$;

- Over $\mathbb{R} \times S^{1} \backslash\left([-T / 2, T / 2] \times S^{1}\right), u_{a}(s, t)=u(a, t)$.

Let $\tau_{L}$ denote translation by $L$ :

$$
\tau_{L} w(s):=w(s-L) .
$$

Definition (Chain topology on $\hat{\mathcal{M}}_{P}^{+}, \hat{\mathcal{M}}_{O}^{+}$). The chain topology of $\hat{\mathcal{M}}_{P}^{+}$ and $\hat{\mathcal{M}}_{O}^{+}$are, respectively, the topology with neighborhood base given by the subsets $N_{P}\left(U_{1}, \ldots, U_{k} ; \Lambda\right)$ and $N_{O}\left(U_{1}, \ldots, U_{k} ; \Lambda\right), N_{O}(U)$ defined below:

(a) Given $\left\{\hat{u}_{1}, \ldots, \hat{u}_{k}\right\} \in \hat{\mathcal{M}}_{P}^{+}(x, y)$, let $u_{i}$ be the centered representative of $\hat{u}_{i}$, and $U_{i}$ be a neighborhood of $u_{i}$ in $L_{1, \text { loc }}^{p}\left(\mathbb{R} \times S^{1} ; p_{2}^{*} T_{f}\right)$. Let $\Lambda \in \mathbb{R}^{+}$, and $N_{P}\left(U_{1}, \ldots, U_{k} ; \Lambda\right) \subset \hat{\mathcal{M}}_{P}^{+}(x, y)$ be the subset consisting of broken trajectories $\left\{\hat{v}_{1}, \ldots, \hat{v}_{m}\right\}$ such that:

- $\exists L_{i} \in \mathbb{R} \forall i$, and a surjective map $j:\{1, \ldots, k\} \rightarrow\{1, \ldots, m\}$, so that $\tau_{-L_{i}} v_{j(i)} \in U_{i}$, where $v_{j}$ is the centered representative of $\hat{v}_{j}$;

- The map $j$ preserves the partial ordering. Namely, $j(i+1)=$ $j(i)+1$ or $j(i)$, and in the latter case, $L_{i+1}-L_{i}>\Lambda$.

(b) When $\left\{\hat{u}_{1}, \ldots, \hat{u}_{k}\right\}$ is a $k$-th broken orbit with $k>0$, and $U_{1}, \ldots, U_{k}, \Lambda$ be defined as in part (a), the subset $N_{O}\left(U_{1}, \ldots, U_{k} ; \Lambda\right) \subset \hat{\mathcal{M}}_{O}^{+}$ consists of:

- broken orbits $\left\{\hat{v}_{1}, \ldots, \hat{v}_{m}\right\}$ with $m>0$ satisfying similar conditions as in part (a) above, but with $j$ now mapping from the indexing set of $\left\{\hat{u}_{1}, \ldots, \hat{u}_{k}\right\}$ to the indexing set of $\left\{\hat{v}_{1}, \ldots, \hat{v}_{m}\right\}$, which preserves partial cyclic ordering, or

- closed orbits $(T, \hat{v})$ with the property that $\exists L_{i} \in \mathbb{R} \forall i$ and $a \in S^{1}$, so that $\tau_{-L_{i}} v_{a} \in U_{i} \forall i, T>k \Lambda$, and $L_{i+1}-L_{i}>\Lambda$ for $i=1, \ldots, k-1$.

When $\left(T_{u}, \hat{u}\right)$ is a closed orbit, and $U$ is a neighborhood of $u_{b}$ in $L_{1, \text { loc }}^{p}\left(\mathbb{R} \times S^{1} ; p_{2}^{*} T_{f}\right)$ for some $b \in S^{1}$, the subset $N_{O}(U) \subset \hat{\mathcal{M}}_{O}^{+}$consists of closed orbits $(T, \hat{v})$ such that $v_{a} \in U$ for some $a \in S^{1}$.

The stronger $\mathcal{B}$-topology on $\mathcal{M}_{P}(x, y)$ and $\mathcal{M}_{O}$ in this Floer theory will be introduced in Sections 3.2.3 and 3.3.1.

3.1.4. The Conley-Zehnder index. Let $A_{x}$ denote the linearization of $\mathcal{V}_{X}$ at $x \in \mathcal{P}(X)$. The critical point $x$ is said to be non-degenerate if $A_{x}$ is surjective. In this case, any lift of $x$ in a covering of $\mathcal{C}$ is said to be non-degenerate. 
Recall that the Conley-Zehnder index assigns continuously an integer $\mathrm{CZ}(A)$ to each surjective operator $A \in \Sigma_{C}$, where $\Sigma_{C}$ is the space of operators $A: L_{1}^{2}\left(S^{1}, \mathbb{R}^{2 n}\right) \rightarrow L^{2}\left(S^{1}, \mathbb{R}^{2 n}\right)$ of the form $A=J_{0} d / d t+\nu(t)$, where $\mathbb{R}^{2 n}$ is equipped with the standard symplectic structure and complex structure $J_{0}$, and $\nu$ is a $C_{\epsilon}$-function taking values in the space of self-adjoint matrices. Furthermore, the spectral flow of a path of such operators $A(s)$, $s \in[0,1]$ is the difference in the Conley-Zehnder indices of $A(1)$ and $A(0)$, and $\mathrm{CZ}\left(J_{0} d / d t\right)=0$. (See e.g., $[\mathbf{3 9}]$. Our $\mathrm{CZ}=\mu_{H}=n-\mu_{\mathrm{CZ}}$ in $[\mathbf{3 9}]$ ).

Let $\hat{\mathcal{C}}$ denote the universal covering of $\mathcal{C}$ and $\hat{\mathcal{P}}$ be the lift of $\mathcal{P}$ in $\hat{\mathcal{C}}$.

Definition. Fix a unitary trivialization of $\gamma_{0}^{*} K$. Together with a homotopy class of paths $w$ from $\gamma_{0}$ to $x$, this trivialization of $\gamma_{0}^{*} K$ induces a (homotopy class of ) unitary trivialization $\Phi_{x,[w]}: x^{*} K \rightarrow S^{1} \times \mathbb{C}^{n}$, for each $(x,[w]) \in \hat{\mathcal{P}}$. The operator $\mathbb{A}_{(x,[w])}:=\left(\Phi_{x,[w]}\right)_{*} A_{x}\left(\Phi_{x,[w]}\right)_{*}^{-1}$ is contained in $\Sigma_{C}$, and it is surjective when $(x,[w])$ is non-degenerate. In this case, define

$$
\hat{\operatorname{ind}}(x,[w])=\mathrm{CZ}\left(\mathbb{A}_{(x,[w])}\right) \in \mathbb{Z} .
$$

A well-known index computation shows that if $(x,[w]),\left(x,\left[w^{\prime}\right]\right) \in \hat{\mathcal{P}}$ are two different lifts of the same $x \in \mathcal{P}$, then

$$
\hat{\operatorname{ind}}(x,[w])-\hat{\operatorname{ind}}\left(x,\left[w^{\prime}\right]\right)=2 c_{1}^{f}\left(\operatorname{im}\left[w-w^{\prime}\right]\right),
$$

which factors through im. Thus, ind descends to define a $\mathbb{Z}$-valued index ind for non-degenerate critical points in $\tilde{\mathcal{C}}$, with SF-homomorphism given by $2 \psi_{c}$. This in turn defines an absolute $\mathbb{Z} / 2 \mathbb{Z}$-valued grading ind for nondegenerate critical points in $\mathcal{C}$, and a relative $\mathbb{Z}$-valued grading on $\tilde{\mathcal{P}}_{\text {ndg }}$, which satisfy the requirements in Section 2.1.

3.1.5. Varying $f$ and varying $(J, X)$. We now describe a well-known and very useful observation.

We'll write $X=X_{f}, K=K_{f}$ to emphasize their dependence on $f$.

First, notice that a diffeotopy from $f$ to $f^{\prime}$ induces a diffeomorphism from $\mathcal{L}_{f} M$ to $\mathcal{L}_{f^{\prime}} M$. In particular, let $\Phi=\left\{\phi_{t} \mid t \in[0,1]\right\}$ be a symplectic isotopy connecting the identity map to $\phi_{1}$, where $f^{\prime}=\phi_{1} \circ f$, and let $\vartheta_{t}$ be the path of closed 1-forms so that $X_{\vartheta_{t}}$ generates $\phi_{t}$. For $\gamma \in \Omega_{\gamma_{0}}(M ; f)$, let

$$
\Phi \cdot \gamma(t):=\phi_{t}(\gamma(t))
$$

This defines an isomorphism

$$
\Phi: \Omega_{\gamma_{0}}(M ; f) \longrightarrow \Omega_{\Phi \cdot \gamma_{0}}\left(M ; f^{\prime}\right) .
$$

It also induces an action of $\pi_{1}\left(\operatorname{Symp}_{0}(M)\right)$ on $\pi_{0}\left(\mathcal{L}_{f} M\right)$.

Suppose further that the path $t \mapsto X_{\vartheta_{t}}$ is in $X_{f}$. (This can always be arranged by e.g., a suitable reparametrization of the path so that 
$\left.X_{\vartheta_{0}}=0=X_{\vartheta_{1}}\right)$. It is easy to see that for any $X \in X_{f}$, there is an $X^{\prime} \in X_{f^{\prime}}$ such that

$$
\begin{aligned}
\Phi^{*} y_{X^{\prime}} & =y_{X} \quad \text { and } \\
\phi_{t}^{*} \theta_{X^{\prime}, t} & :=\theta_{X, t}+\vartheta_{t} .
\end{aligned}
$$

Furthermore, if $u(s, t)$ solves (15), then

$$
w(s, \cdot):=\Phi \cdot u(s, \cdot)
$$

also satisfies (15), but with $f$ there replaced by $f^{\prime}, X$ replaced by $X^{\prime}$, and $J_{t}$ replaced by $J_{t}^{\prime}$, where

$$
J_{t}^{\prime}\left(\phi_{t}(x)\right):=\left(D \phi_{t}\right) J_{t}(x)\left(D \phi_{t}\right)^{-1} .
$$

(Notice that $J^{\prime} \in \mathcal{J}_{K_{f}^{\prime}}$ if $J \in \mathcal{J}_{K_{f}}$ ).

This not only defines isomorphisms between the spaces $\mathcal{P}^{\gamma_{0}}(M, f ; X)$, $\mathcal{M}_{P}^{\gamma_{0}}(M, f ; J, X), \mathcal{M}_{O}^{\gamma_{0}}(M, f ; J, X)$ and $\mathcal{P}^{\Phi \cdot \gamma_{0}}\left(M, f^{\prime} ; X^{\prime}\right), \mathcal{M}_{P}^{\Phi \cdot \gamma_{0}}\left(M, f^{\prime} ; J^{\prime}, X^{\prime}\right)$, $\mathcal{M}_{O}^{\Phi \cdot \gamma_{0}}\left(M, f^{\prime} ; J^{\prime}, X^{\prime}\right)$, respectively, but also equivalences of the relevant deformation operators $\left(A_{x}\right.$, and $E_{u}, \tilde{D}_{u}$ in Sections 3.2 and 3.3$)$ by similarity transformations. Thus the Floer theories associated to $\left(M, f^{\prime}, \Phi \cdot \gamma_{0} ; J^{\prime}, X^{\prime}\right)$ and $\left(M, f, \gamma_{0} ; J, X\right)$ and are completely equivalent.

Because of this equivalence, in this paper we shall fix the symplectomorphism $f$ and vary the almost complex structure $J$ and the symplectic vector field $X$.

3.2. Structure of the moduli spaces: $\mathcal{P}, \mathcal{M}_{P}$. The goal of the rest of this section is to show that for regular $(J, X)$ (to be defined in Section 3.3), the associated formal flow is a Floer system.

Since the results in this subsection all follow from simple adaptations of the literature, we shall omit most details except the notions needed for later sections.

For the rest of this section, we fix a $J \in \mathcal{J}_{K}^{\text {reg }}$ and perturb $X \in \mathcal{X}$ by Hamiltonian vector fields to achieve transversality.

3.2.1. The space $\mathcal{P}$. Recall the definition of $M_{c}\left(J_{t}\right), M_{c}(J)$ from the end of Section 3.1.2.

Definition. Given $J \in \mathcal{J}_{K}^{\text {reg }}$, we say that $X \in X$ is $J$-non-degenerate if the following hold:

(a) $\mathcal{P}(X)$ consists of finitely many non-degenerate points.

(b) For any $\gamma \in \mathcal{P}(X), \gamma(t) \notin M_{1}\left(J_{t}\right) \forall t$.

Proposition. Given $J \in \mathcal{J}_{K}^{\text {reg }}$ and $X \in \mathcal{X}$, there is a Baire set $\mathcal{H}^{\text {ndg }}(J, X) \subset$ $\mathcal{H}$ such that $X+\chi_{H}$ is $J$-non-degenerate for all $H \in \mathcal{H}^{\text {ndg }}(J, X)$. 
Proof. These follow from simple adaptations of [20] Theorem 3.1, using Lemma 3.1.2(a) above. For part (b), we replace the evaluation map in [20] by

$$
M_{1}(J) \times \mathcal{P}^{\mathcal{H}}(X) \rightarrow T_{f} \times_{S^{1}} T_{f}:(q,(x, H)) \mapsto\left(q, x\left(\pi_{S}(q)\right)\right),
$$

where $\mathcal{P}^{\mathcal{H}}(X)=\bigcup_{H \in \mathcal{H}} \mathcal{P}\left(X+\chi_{H}\right)$ is the universal moduli space of critical points that fibers over $\mathcal{H}$, and $(x, H)$ denotes an element in this universal moduli space: $x \in \mathcal{P}\left(X+\chi_{H}\right)$.

In particular, this means that given a fixed pair $(J, X) \in \mathcal{J}_{K}^{\mathrm{reg}} \times \mathcal{X}$, the pair $\left(J, X+\chi_{H}\right)$ satisfies (FS1) for all $H \in \mathcal{H}^{\text {ndg }}(J, X)$.

3.2.2. The Space $\mathcal{M}_{P}$. We now show that (FS2) holds for "generic" $(J, X)$, in a sense we clarify next.

Consider Hamiltonian perturbations in the following space:

Definition. Let $J \in \mathcal{J}_{K}^{\text {reg }}$ and let $X \in \mathcal{X}$ be $J$-non-degenerate. Let $\delta \in \mathbb{R}^{+}$ and $k \in \mathbb{Z}^{+}$. Then

$$
\begin{aligned}
& V_{\delta}^{k}(J, X) \\
& \quad:=\left\{H \mid H \in \mathcal{H},\|H\|_{C_{\epsilon}} \leq \delta ; \nabla^{i} H_{t}(x(t))=0 \quad \forall x \in \mathcal{P}(X), \quad i=0, \ldots, k\right\} .
\end{aligned}
$$

Notice that $\mathcal{P}\left(X+\chi_{H}\right)=\mathcal{P}(X) \forall H \in V_{\delta}^{k}(J, X)$.

Given $J \in \mathcal{J}_{K}^{\mathrm{reg}}$, an element $u$ of $\mathcal{M}_{P}$ or $\mathcal{M}_{O}$ is said to be $J$-regular if in addition to being non-degenerate, it also satisfies:

$$
u(\cdot, t) \cap M_{0}\left(J_{t}\right)=\emptyset \quad \forall t .
$$

A moduli space (any variant of $\mathcal{M}_{P}$ or $\mathcal{M}_{O}$ ) is said to be non-degenerate if it consists of non-degenerate elements; it is $J$-regular if it consists of $J$-regular elements.

Proposition. Let $J, X$ be as in the previous Definition. Then for any $k \geq 2$ and any small positive number $\delta$, there is a Baire set $V_{\delta}^{k, \operatorname{Preg}}(J, X) \subset$ $V_{\delta}^{k}(J, X)$ such that all $H \in V_{\delta}^{k, \operatorname{Preg}}(J, X)$ satisfies:

(a) (FS2) holds for $\left(J, X+\chi_{H}\right)$;

(b) $\mathcal{M}_{P}^{i}\left(x, y ; J, X+\chi_{H}\right)$ is $J$-regular for any $i \leq 2, x, y \in \mathcal{P}$.

Proof. These again follow from simple adaptations of [20]. Transversality follows the arguments of [20] Theorem 3.2, using again Lemma 3.1.2, and replacing the evaluation map by

$$
\left.M_{0}(J) \times \mathcal{M}_{P}^{i, V_{\delta}^{k}}(x, y) ; J, X\right) \longrightarrow T_{f} \times_{S^{1}} T_{f}:(q,(u, H)) \mapsto\left(q, u\left(0, \pi_{S}(q)\right)\right),
$$

where $\mathcal{M}_{P}^{i, V_{\delta}^{k}}(x, y ; J, X)=\bigcup_{H \in V_{\delta}^{k}(J, X)} \mathcal{M}_{P}^{i}\left(x, y ; J, X+\chi_{H}\right)$ is the universal moduli space which fibers over $V_{\delta}^{k}(J, X)$, the term $(u, H)$ denotes an element over $H \in V_{\delta}^{k}(J, X)$ in $\mathcal{M}_{P}^{i, V_{\delta}^{k}}(x, y ; J, X)$, and $u \in \mathcal{M}_{P}\left(x, y ; J, X+\chi_{H}\right)$. 
The compactness follows the argument of $[\mathbf{2 0}]$ Theorem 3.3. The main ingredients are: Gromov compactness, $J$-non-degeneracy of $X+\chi_{H}$, and statement (b) in the Proposition proven by the transversality argument above. The well-known fact that $u$ decays exponentially near the nondegenerate critical points $x, y$ (see e.g., $[\mathbf{1 2}]$ p. 607, [10] pp. 801-803) is also used.

3.2.3. The configuration space $\mathcal{B}_{P}$, and the deformation operator $E_{u}$. We now introduce the configuration space $\mathcal{B}_{P}$, which endows $\mathcal{M}_{P}$ with the $\mathcal{B}$-topology, and the relevant deformation operator. These notions are used in the omitted details of the proof of Proposition 3.2.2, and shall also be needed in later sections.

Let $\Theta:=\mathbb{R} \times S_{1}^{1}$; and recall that $p_{2}: \Theta \rightarrow S_{1}^{1}$ is the projection to the second factor.

Let $\beta: \mathbb{R} \rightarrow[0,1]$ be a smooth cutoff function supported on $\mathbb{R}^{+}$such that $\beta(s)=1$ as $s \geq 1$.

Given $J \in \mathcal{J}_{K}$, we define a $t$-dependent exponential map exp $: T \mathcal{C} \rightarrow \mathcal{C}$,

$$
\exp (x, \xi)(t):=\left(\exp _{x(t)}^{g_{t}} \xi(t), t\right),
$$

where $\exp _{x(t)}^{g_{t}}$ is the exponential map in the fiber $M$ with respect to $g_{t}$, the metric on $M$ corresponding to $J_{t} . \xi \in L_{1}^{p}\left(x^{*} K\right)=T_{x} \mathrm{e}$.

Definition. Suppose $V$ is an Euclidean or Hermitian vector bundle over $\Theta ; \sigma_{-}, \sigma_{+} \in \mathbb{R}$. Let $L_{k:\left(\sigma_{-}, \sigma_{+}\right)}^{p}(V)$ be the (exponentially) weighted Sobolev space:

$$
\left\{\xi \mid \varsigma^{\sigma_{-} \sigma_{+}} \xi \in L_{k}^{p}\right\} \text {, where } \varsigma^{\sigma_{-} \sigma_{+}}(s):=e^{-\left(s \beta(s) \sigma_{+}+s \beta(-s) \sigma_{-}\right)} .
$$

Let $p_{2}^{*} T_{f} \rightarrow \Theta$ be the pullback bundle of $T_{f} \rightarrow S^{1}$. Given two critical points $x, y \in \mathcal{P}(X)$, the configuration space is

$$
\begin{aligned}
& \mathcal{B}_{P}^{\left(\sigma_{1}, \sigma_{2}\right)}(x, y):= \\
& \left\{\begin{array}{ll}
u & \begin{array}{l}
u \in L_{1, l o c}^{p}\left(\Theta, p_{2}^{*} T_{f}\right), \\
u(s, \cdot)=\exp \left(y, \xi_{+}(s, \cdot)\right) \text { for some } \xi_{+} \in L_{1:\left(0, \sigma_{2}\right)}^{p}\left(p_{2}^{*}\left(y^{*} K\right)\right) \text { if } s>\rho_{+}(u), \\
u(s, \cdot)=\exp \left(x, \xi_{-}(s, \cdot)\right) \text { for some } \xi_{-} \in L_{1:\left(\sigma_{1}, 0\right)}^{p}\left(p_{2}^{*}\left(x^{*} K\right)\right) \text { if } s<\rho_{-}(u)
\end{array}
\end{array}\right\},
\end{aligned}
$$

where $\rho_{ \pm} \in \mathbb{R}$ are numbers depending on $u$. The integer $p>2$.

Understood as the space of paths in $\mathcal{C}$ between the two points $x, y$, $\mathcal{B}_{P}(x, y)$ decomposes into many components according to the homology classes of the paths, like $\mathcal{M}_{P}(x, y)$. Thus, we may define $\mathcal{B}_{P}((x,[w]),(y,[v]))$, $\mathcal{B}_{P}^{k}(x, y)$ in complete analogy to $\mathcal{M}_{P}((x,[w]),(y,[v])), \mathcal{M}_{P}^{k}(x, y)$.

The space $\mathcal{B}_{P}^{\left(\sigma_{1}, \sigma_{2}\right)}(x, y)$ is a Banach manifold (see e.g., the argument of Theorem 3 in $[\mathbf{1 0}])$. The local model of a neighborhood of $u$ is $L_{1:\left(\sigma_{1}, \sigma_{2}\right)}^{p}\left(u^{*} K\right)$ 
via the $t$-dependent exponential map

$$
\exp (u, \xi):=\left(\exp _{u(s, t)}^{g_{t}}(\xi(s, t)),(s, t)\right) .
$$

The precise definition of weighted moduli space is:

$$
\mathcal{M}_{P}^{\left(\sigma_{1}, \sigma_{2}\right)}(x, y ; J, X):=\mathcal{M}_{P}(J, X) \cap \mathcal{B}_{P}^{\left(\sigma_{1}, \sigma_{2}\right)}(x, y) .
$$

Notice that we do not require any non-degeneracy condition on $x, y$ for the definitions of $\mathcal{B}_{P}^{\left(\sigma_{1}, \sigma_{2}\right)}(x, y)$ and $\mathcal{M}_{P}^{\left(\sigma_{1}, \sigma_{2}\right)}(x, y ; J, X)$. However, with suitable non-degeneracy conditions on $x, y, \mathcal{M}_{P}^{\left(\sigma_{1}, \sigma_{2}\right)}(x, y ; J, X)$ admits a description as the zero locus of the Fredholm section $\bar{\partial}_{J X}$ of a Banach bundle. (cf. e.g., $[\mathbf{1 0}])$. See the next Lemma.

Let $E_{u}^{\left(\sigma_{1}, \sigma_{2}\right)}: L_{1:\left(\sigma_{1}, \sigma_{2}\right)}^{p}\left(u^{*} K\right) \rightarrow L_{:\left(\sigma_{1}, \sigma_{2}\right)}^{p}\left(u^{*} K\right)$ denote the linearization of $\bar{\partial}_{J X}$ at $u$.

In this Floer theory, the weighted versions of gradings are defined via ind $^{\sigma}$ :

$$
\begin{gathered}
\operatorname{ind}^{\sigma}(x)=\tilde{\operatorname{ind}}^{\sigma}(x,[w]) \quad \bmod 2 \\
\operatorname{gr}^{\left(\sigma_{1}, \sigma_{2}\right)}\left(\left(x_{1},\left[w_{1}\right]\right),\left(x_{2},\left[w_{2}\right]\right)\right)=\text { ind }^{\sigma_{1}}\left(x_{1},\left[w_{1}\right]\right)-\text { ind }^{\sigma_{2}}\left(x_{2},\left[w_{2}\right]\right),
\end{gathered}
$$

where ind $^{\sigma}$ is the generalized Conley-Zehnder index, defined as follows. Given $\sigma \in \mathbb{R}$, we say that an $x \in \mathcal{P}(X)$ is $\sigma$-weighted non-degenerate if $A_{x}+\sigma$ is surjective. In this case,

$$
\operatorname{ind}^{\sigma}(x,[w]):=\tilde{\operatorname{ind}}_{\gamma_{0}}^{\sigma}(x,[w]):=\mathrm{CZ}\left(\mathbb{A}_{(x,[w])}+\sigma\right) .
$$

A routine modification of the literature (e.g., $[\mathbf{1 2}],[\mathbf{3 9}],[\mathbf{4 0}])$ yields the next lemma.

Lemma. Let $u \in \mathcal{M}_{P}((x,[z]),(y,[w]) ; J, X)$, and suppose that $x, y \in \mathcal{P}(X)$ are $\sigma_{1}$-weighted non-degenerate and $\sigma_{2}$-weighted non-degenerate, respectively. Then the operator

$$
E_{u}^{\left(\sigma_{1}, \sigma_{2}\right)}: L_{1:\left(\sigma_{1}, \sigma_{2}\right)}^{p}\left(u^{*} K\right) \rightarrow L_{:\left(\sigma_{1}, \sigma_{2}\right)}^{p}\left(u^{*} K\right)
$$

is Fredholm of index $\operatorname{ind}^{\sigma_{1}}(x,[w])-\operatorname{ind}^{\sigma_{2}}(y,[v])$.

In fact, the moduli space $\mathcal{M}_{P}^{\left(\sigma_{1}, \sigma_{2}\right)}(x, y)$ is independent of small perturbations to the weights $\sigma_{1}, \sigma_{2}$ When $x, y$ are $\sigma_{1}$-weighted non-degenerate and $\sigma_{2}$-weighted non-degenerate, respectively.

Notation. By standard decay estimates, $\mathcal{M}_{P}(x, y)=\mathcal{M}_{P}^{(0,0)}(x, y)$. Thus, we shall omit the superscripts $\left(\sigma_{1}, \sigma_{2}\right)$ when the weights $\left(\sigma_{1}, \sigma_{2}\right)=(0,0)$, and the critical points $x, y$ are non-degenerate. 
3.3. Structure of the moduli spaces: $\mathcal{M}_{O}$. We now verify the genericity of the condition (FS3); more precisely, we prove:

Proposition. Given $J \in \mathcal{J}_{K}^{\text {reg }}$, a $J$-non-degenerate $X \in X$, an integer $k \geq 2$, and a small $\delta \in \mathbb{R}^{+}$, the set

$$
V_{\delta}^{k, \mathrm{reg}}(J, X):=\left\{H \mid H \in V_{\delta}^{k}(J, X), \text { (FS2), (FS3) hold for }\left(J, X+\chi_{H}\right)\right\}
$$

is Baire in $V_{\delta}^{k}(J, X)$.

Notice that by assumption, (FS1) already holds for $\left(J, X+\chi_{H}\right)$, for all $H \in V_{\delta}^{k}(J, X)$. We call $(J, X)$ a regular pair if $J \in \mathcal{J}_{K}^{\text {reg }}, X=X_{0}+\chi_{H}$, where $X_{0}$ is $J$-non-degenerate and $H \in V_{\delta}^{k, \text { reg }}\left(J, X_{0}\right)$. Since the properties (FS1), (FS2), (FS3) hold for regular pairs, $\tau_{\mathrm{F}}(J, X), \zeta_{\mathrm{F}}(J, X), I_{\mathrm{F}}(J, X)$ are welldefined for regular pairs. Regular pairs are generic in the sense described in this section.

This proposition follows from the combination of Lemmas 3.3.2, 3.3.3, 3.3.4 below: $V_{\delta}^{k, \text { reg }}(J, X)$ is Baire since it contains $\bigcap_{\Re \in \mathbb{R}^{+}} V_{\delta}^{k, \Re \text {-reg }}(J, X)$, where $V_{\delta}^{k, \Re \text {-reg }}(J, X)$ are the open dense sets in Lemma 3.3.4. We first introduce some basic notions before stating these lemmas.

3.3.1. The configuration space $\mathcal{B}_{O}$ and the deformation operator $\tilde{D}_{(T, u)}$. Because we include closed orbits of all periods in $\mathcal{M}_{O}$, we shall often write an element of $\mathcal{M}_{O}$ as a pair $(T, u)$, where $T \in \mathbb{R}^{+}$is the period of the closed orbit, and $u$ is a section of the $M$-bundle $p_{2}^{*} T_{f}$ over $S_{T}^{1} \times S_{1}^{1}$, where $p_{2}: S_{T}^{1} \times S_{1}^{1} \rightarrow S_{1}^{1}$ is again the projection to the second factor.

The configuration space $\mathcal{B}_{O}$ for $\mathcal{M}_{O}$ is then

$$
\mathcal{B}_{O}:=\left\{(T, u) \mid \begin{array}{c}
T \in \mathbb{R}^{+} ; u \in L_{1}^{p}\left(S_{T}^{1} \times S_{1}^{1}, p_{2}^{*} T_{f}\right) \\
\text { s.t. }\left.u\right|_{\{0\} \times S_{1}^{1}} \text { is homotopic to } \gamma_{0}
\end{array}\right\} .
$$

It is easy to see that $\mathcal{B}_{O}$ is a Banach manifold modeled on $\mathbb{R} \times L_{1}^{p}\left(u^{*} K\right)$, and it fibers over $\mathbb{R}^{+}$by mapping each $(T, u) \in \mathcal{B}_{O}$ to $T$. $\mathcal{M}_{O}(J, X)$ embeds in $\mathcal{B}_{O}$ as the zero locus of the Fredholm section $\bar{\partial}_{J X}$ of a Banach bundle.

Let $\tilde{D}_{(T, u)}$ be the linearization of $\bar{\partial}_{J X}$ at $(T, u) \in \mathcal{M}_{O}(J, X)$. It has the following expression:

$$
\tilde{D}_{(T, u)}(\varrho, \xi)=D_{u} \xi-\varrho / T \partial_{s} u \quad \text { for }(\varrho, \xi) \in \mathbb{R} \times L_{1}^{p}\left(u^{*} K\right),
$$

where the operator $D_{u}: L_{1}^{p}\left(u^{*} K\right) \rightarrow L^{p}\left(u^{*} K\right)$ is given by the same formula as $E_{u}$, but here $s$ takes value in $S_{T}^{1}$ instead of $\mathbb{R}$.

$\tilde{D}_{(T, u)}$ fits into the deformation complex:

$$
\mathbb{R} \stackrel{d_{u}}{\longrightarrow} \mathbb{R} \oplus L_{1}^{p}\left(u^{*} K\right) \stackrel{\tilde{D}_{(T, u)}}{\longrightarrow} L^{p}\left(u^{*} K\right)
$$

where $d_{u}$ is the linearization of the $S^{1}$ action (by translation in $s$ ) on $\mathcal{M}_{O}$,

$$
d_{u}(\lambda)=\left(0, \lambda \partial_{s} u\right) .
$$


It is useful to combine $d_{u}$ and $\tilde{D}_{(T, u)}$ into the following operator $\mathcal{D}_{(T, u)}$, which is deformation operator of the reduced moduli space $\hat{\mathcal{M}}_{O}=\mathcal{M}_{O} / S^{1}$ :

$$
\begin{aligned}
& \mathcal{D}_{(T, u)}= \mathcal{D}_{(T, u)}^{J, X}: \mathbb{R} \oplus L_{1}^{p}\left(u^{*} K\right) \longrightarrow \mathbb{R} \oplus L^{p}\left(u^{*} K\right), \\
& \mathcal{D}_{(T, u)}=\tilde{D}_{(T, u)}+d_{u}^{*}=\left(\begin{array}{cc}
0 & \Pi_{\partial_{s} u} \\
-1 / T \partial_{s} u & D_{u}
\end{array}\right),
\end{aligned}
$$

where $\Pi_{\partial_{s} u}$ denotes $L^{2}$-orthogonal projection to $\partial_{s} u$, and $d_{u}^{*}$ is the formal $L^{2}$-adjoint of $d_{u}$.

The superscripts $J, X$ are added to the operators when we wish to emphasize their dependence on $J, X$.

$\mathcal{D}_{(T, u)}$ is obviously Fredholm, since $D_{u}$ is elliptic. By Riemann-Roch

$$
\operatorname{ind}\left(\mathcal{D}_{(T, u)}\right)=\operatorname{ind}\left(D_{u}\right)=2 \psi_{c}(A) \quad \text { when }(T, u) \in \mathcal{M}_{O}(A) .
$$

Notice that the non-degeneracy of a $(T, u) \in \mathcal{M}_{O}$ is equivalent to the non-degeneracy of its image in $\hat{\mathcal{M}}_{O}$ : Since a closed orbit is by definition non-constant in $s, d_{u}$ is always injective, and thus the surjectivity of $\tilde{D}_{(T, u)}$ and $\mathcal{D}_{(T, u)}$ are equivalent.

The standard transversality argument works (only) for the space of simple closed orbits, which we denote by $\mathcal{M}_{O}^{d, \text { sim }} \subset \mathcal{M}_{O}^{d}$.

3.3.2. Lemma. Let $(J, X)$ be as in the Proposition. Then the subset

$$
\left\{H \mid H \in V_{\delta}^{k}(J, X), \mathcal{M}_{O}^{\mathrm{sim}}\left(J, X+\chi_{H}\right) \text { is non-degenerate }\right\}
$$

is Baire in $V_{\delta}^{k}(J, X)$. So is the subset

$$
\left\{H \mid H \in V_{\delta}^{k}(J, X), \coprod_{l \leq 1} \mathcal{M}_{O}^{l, \operatorname{sim}}\left(J, X+\chi_{H}\right) \text { is } J \text {-regular }\right\} .
$$

Proof. Let $\mathcal{M}_{O}^{V_{\delta}^{k}, \operatorname{sim}}(J, X)=\bigcup_{H \in V_{\delta}^{k}(J, X)} \mathcal{M}_{O}^{\operatorname{sim}}\left(J, X+\chi_{H}\right)$ be the universal moduli space which fibers over $V_{\delta}^{k}(J, X)$. We want to show that it is nondegenerate, namely, for any $((T, u), H) \in \mathcal{M}_{O}^{V_{\delta}^{k}, \operatorname{sim}}(J, X)$, the operator

$$
\begin{array}{r}
\tilde{D}_{((T, u), H)}^{V_{\delta}^{k}}: \mathbb{R} \oplus L_{1}^{p}\left(u^{*} K\right) \oplus V_{\delta}^{k}(J, X) \rightarrow L^{p}\left(u^{*} K\right), \\
\tilde{D}_{((T, u), H)}^{V_{\delta}^{k}}((\varrho, \xi), h)=\tilde{D}_{(T, u)}^{J, X+\chi_{H}}(\varrho, \xi)+\nabla h
\end{array}
$$

is surjective. Equivalently, letting $D_{u}^{*}$ denote the formal $L^{2}$-adjoint of $D_{u}$, we want to show that there is no $\eta$ such that

$$
\begin{aligned}
D_{u}^{*} \eta & =0, \\
\left\langle\partial_{s} u, \eta\right\rangle_{2} & =0, \\
\langle\nabla h, \eta\rangle_{2} & =0 \quad \forall h .
\end{aligned}
$$


To apply the standard transversality argument ${ }^{2}$, we need the following observations: first, by unique continuation (e.g., [14] Proposition 3.1) the set

$$
\Omega(u):=\{(s, t) \mid u(s, t)=x(t) \text { for some } x \in \mathcal{P}(X)\} \subset S_{T}^{1} \times S_{1}^{1}
$$

consists of discrete points for any $u \in \mathcal{M}_{O}\left(J, X+\chi_{H}\right)$; also, for simple $u$ the argument of $[\mathbf{1 4}]$ Proposition 4.1 shows that the set

$$
R(u)=\left\{(s, t) \mid(s, t) \notin \Omega(u), u(s, t) \neq u\left(s^{\prime}, t\right) \forall t, s \neq s^{\prime}\right\} \subset S_{T}^{1} \times S_{1}^{1}
$$

is open dense. One may then follow the standard arguments (e.g., in the proof of Theorem 5.1 (i) of $[\mathbf{1 4}]$ ) to show that $\eta=g(t) \partial_{s} u$ for some realvalued function $g(t)$. However, from (18), we have

$$
\int g(t)\left|\partial_{s} u(s, t)\right|^{2} d s d t=0
$$

This contradicts with the fact that $g(t) \neq 0 \forall t$ and $\partial_{s} u(s, t) \neq 0$ for all but discrete $s, t$ (cf. [14]). To show that the set (17) is also Baire, consider in addition the evaluation map

$$
\begin{aligned}
e v: M_{0}(J) \times \mathcal{M}_{O}^{V_{\delta}^{k}, l, \operatorname{sim}}(J, X) & \rightarrow T_{f} \times_{S^{1}} T_{f}, \\
(y,((T, u), H)) & \mapsto\left(y, u\left(0, \pi_{S}(y)\right)\right) .
\end{aligned}
$$

This can be seen to be transverse to the diagonal by the usual argument; so a generic fiber of $e v^{-1}(\mathrm{diag})$ is a manifold of dimension $2 n-3+1+l-2 n=$ $l-2<0$ - hence empty. $\leq \Re$.

Let $\mathcal{M}_{O}^{1, \operatorname{sim}, \Re} \subset \mathcal{M}_{O}^{1, \text { sim }}$ be the subspace of simple closed orbits with energy

3.3.3. Lemma. Let $J \in J_{K}^{\mathrm{reg}}, X$ be $J$-non-degenerate, and $\Re \in \mathbb{R}^{+}$be arbitrary. If $\mathcal{M} \leq 1, \Re(J, X):=\coprod_{l \leq 1}\left(\mathcal{M}_{O}^{l, \Re}(J, X) \cup \mathcal{M}_{P}^{l, \Re}(J, X)\right)$ is J-regular, then the reduced moduli space $\hat{\mathcal{M}}^{0} \Re(J, X):=\hat{\mathcal{M}}_{O}^{0, \Re}(J, X) \cup \hat{\mathcal{M}}_{P}^{0, \Re}(J, X)$ is compact.

Proof. The compactness of $\hat{\mathcal{M}}_{P}^{0, \Re}(J, X)$ was a part of the proof of Proposition 3.2 .2 , we therefore only need to consider $\hat{\mathcal{M}}_{O}^{0, \Re}(J, X)$.

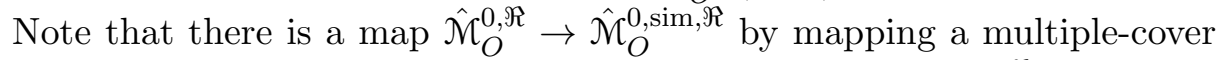
to its underlying simple closed orbit. The fiber of a $u \in \hat{\mathcal{M}}_{O}^{0, \operatorname{sim}, \Re}$ consists of $n(u)$ points, where

$$
n(u):=\left[-\Re / \mathscr{y}_{X}([u])\right] \in \mathbb{Z}^{+}
$$

\footnotetext{
${ }^{2}$ There is vast literature on the transversality proof; unfortunately, many of the papers contain (minor) errors. In [14] author is unaware of errors, and it shall be our main reference.
} 
is bounded above since $-y_{X}([u])$ is bounded below by a positive number. To see this, suppose there is a sequence of closed orbits $\left\{u_{n}\right\}$ with $\mathcal{E}\left(u_{n}\right) \rightarrow 0$ as $n \rightarrow \infty$. By Gromov compactness, there is a subsequence converging in $C_{0}$-norm to a constant flow (i.e., a critical point). This means that the homology class $\left[u_{n}\right]=0$ for large $n$, contradicting the fact that closed orbits have positive energy.

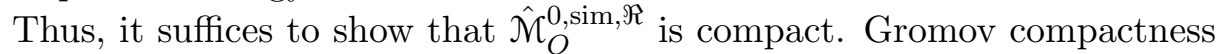
assures us that there are only three ways in which compactness can fail. One is bubbling off spheres; the second is the existence of a sequence of elements in $\hat{\mathcal{M}}_{O}^{0, \operatorname{sim}, \Re}(J, X)$ with periods going to $\infty$, which converge weakly to a broken orbit; the third is the existence of a sequence $\left\{\left(T_{i}, u_{i}\right)\right\} \subset$ $\hat{\mathcal{M}}_{O}^{0, \operatorname{sim}, \Re}(J, X)$ converging to a multiple-cover.

The first possibility is eliminated by the assumption of $J$-regularity. The second possibility is a codimension 1 phenomenon, and is eliminated by the assumption that $\mathcal{M}_{P}^{\leq 1, \Re}(J, X)$ is non-degenerate. In the third case, the multiple cover that the sequence converges to cannot be non-degenerate, contradicting the assumption that $\mathcal{M}_{O}^{\leq 1, \Re}(J, X)$ is non-degenerate.

It remains to show that multiple covers are also generically non-degenerate. For this purpose, notice that to show that $\hat{\mathcal{M}}_{O}^{\Re}$ is non-degenerate, it is equivalent to show that each $u \in \hat{\mathcal{M}}_{O}^{\operatorname{sim}, \Re}$ is " $n(u)$-non-degenerate", $n(u)$ being given by (19):

Let $u \in \hat{\mathcal{M}}_{O}^{\operatorname{sim}}$; we denote the unique $\mathbb{Z} / m \mathbb{Z}$-cyclic cover of $u$ by $u^{m} . u$ is said to be $n$-non-degenerate if $u^{1}, u^{2}, \ldots, u^{n}$ are all non-degenerate.

3.3.4. Lemma. Let $J, X, k, \delta$ be as in the Proposition. Then for each $\Re \in \mathbb{R}^{+}$, the set

$$
V_{\delta}^{k, \Re-\operatorname{reg}}(J, X):=\left\{H \mid H \in V_{\delta}^{k}(J, X), \mathcal{M}^{\leq 1, \Re}\left(J, X+\chi_{H}\right) \text { is J-regular }\right\}
$$

is open and dense in $V_{\delta}^{k}(J, X)$.

Proof. Because by the previous lemma $\mathcal{M} \leq 1, \Re$ consists of finitely many isolated points, the proof is reduced to showing that transversality can be achieved by Hamiltonian perturbations near each $u \in \mathcal{M} \leq, \Re$.

The openness of $V_{\delta}^{k, \Re-\text { reg }}(J, X)$ is obvious from the compactness result of Lemma 3.3.3. To show the denseness, we shall show that for any $H \in$ $V_{\delta}^{k}(J, X)$, we may perturb $H$ by some small $h$ so that $\mathcal{M} \leq 1, \Re\left(J, X+\chi_{H+h}\right)$ is $J$-regular. By Proposition 3.2.2 and Lemma 3.3.2, we may assume without loss of generality that $\mathcal{M}_{\bar{P}}^{\leq 1, \Re}\left(J, X+\chi_{H}\right)$ and $\mathcal{M}_{\bar{O}}^{\leq 1, \operatorname{sim}, \Re}\left(J, X+\chi_{H}\right)$ are already $J$-regular. Adding any sufficiently small $h$ will not affect the $J$-regularity of these moduli spaces. We want to show that $h$ may be chosen to make each $(T, u) \in \hat{\mathcal{M}}_{O}^{1, \operatorname{sim}, \Re} n(u)$-non-degenerate. 
For this purpose, we examine how the cokernel of the deformation operator changes under perturbation, similar to Lemma 5.13 of [44].

Let $m \leq n(u)$ be a positive integer. Recall that the domain of $u^{m}$, denoted $\Theta_{u^{m}}$, is a $\mathbb{Z} / m \mathbb{Z}$-cyclic covering of the domain of $u, \Theta_{u}$ :

$$
c_{m}: \Theta_{u^{m}} \rightarrow \Theta_{u} \text {. }
$$

The group $\mathbb{Z} / m \mathbb{Z}$ acts on the bundle $\left(u^{m}\right)^{*} K$ over $\Theta_{u^{m}}$ by deck transformation. On the other hand, let $\mathbb{R}^{m}$ denote the Euclidean space spanned by the orthonormal basis $\left\{e_{i} \mid i \in \mathbb{Z} / m \mathbb{Z}\right\}$, endowed with a $\mathbb{Z} / m \mathbb{Z}$ action by cyclic permutation of the basis vectors: let $L$ be a generator of $\mathbb{Z} / m \mathbb{Z}$, then $L e_{i}=e_{i+1}$. Consider

$$
V_{m}:=\left(u^{m}\right)^{*} K \otimes_{\mathbb{Z} / m \mathbb{Z}} \mathbb{R}^{m} .
$$

This is an $\mathbb{R}^{2 n m}$-bundle over $\Theta_{u}$, and there is a standard isomorphism

$$
i_{m}: \Gamma\left(\left(u^{m}\right)^{*} K\right) \rightarrow \Gamma\left(V_{m}\right),
$$

via the identification of the sheaf of sections of $V_{m}$ with the direct-image under $c_{m *}$ of the sheaf of sections of $\left(u^{m}\right)^{*} K$.

Instead of $\mathcal{D}_{\left((m T), u^{m}\right)}$, we may equivalently consider the operator ${ }^{m} \mathcal{D}_{(T, u)}$ induced by this isomorphism.

$$
\begin{aligned}
m_{\mathcal{D}_{(T, u)}}: \mathbb{R} \oplus L_{1}^{p}\left(V_{m}\right) & \rightarrow \mathbb{R} \oplus L^{p}\left(V_{m}\right), \\
m_{\mathcal{D}_{(T, u)}} & =\left(\begin{array}{cc}
0 & \Pi_{i_{m}\left(\partial_{s} u^{m}\right)} \\
-1 /(m T) i_{m}\left(\partial_{s} u^{m}\right) & { }^{m} D_{(T, u)}
\end{array}\right) .
\end{aligned}
$$

Suppose that ${ }^{m} \mathcal{D}_{(T, u)}$ has a $p_{m}$-dimensional cokernel, say spanned by $\underline{\eta}_{1}, \underline{\eta}_{2}, \ldots, \underline{\eta}_{p_{m}}$. Then the kernel of ${ }^{m} \mathcal{D}_{(T, u)}$ is also $p_{m}$-dimensional, spanned by $\underline{\xi}_{1}, \underline{\xi}_{2}, \ldots, \underline{\xi}_{p_{m}}$. Note that $\mathbb{Z} / m \mathbb{Z}$ acts orthogonally on the kernel and the cokernel; in fact, the action on the kernel and the cokernel are the same, since the kernel and cokernel both vanish under some deformation of the operator. Furthermore, since by assumption $(T, u)$ is 1-non-degenerate, there is no $\mathbb{Z} / m \mathbb{Z}$-invariant elements in ker $^{m} \mathcal{D}_{(T, u)}$, coker ${ }^{m} \mathcal{D}_{(T, u)}$. Therefore, the bases $\left\{\underline{\xi}_{i}\right\},\left\{\underline{\eta}_{j}\right\}$ may be chosen such that with respect to them, the representations of $\mathbb{Z} / m \mathbb{Z}$ on ker ${ }^{m} \mathcal{D}_{(T, u)}$, coker ${ }^{m} \mathcal{D}_{(T, u)}$ both have the form:

$$
L^{k}:=\bigoplus_{l=1}^{p^{\prime}}\left(\begin{array}{cc}
\cos \left(2 \pi q_{l} k / m\right) & -\sin \left(2 \pi q_{l} k / m\right) \\
\sin \left(2 \pi q_{l} k / m\right) & \cos \left(2 \pi q_{l} k / m\right)
\end{array}\right) \bigoplus(-1)^{\oplus\left(p_{m}-2 p^{\prime}\right)} .
$$

The $(-1)$ components appear only when $m$ is even. We choose the orientation of the bases such that $q_{l} \in\{1,2, \ldots,[(m-1) / 2]\}$.

Let $\left(\text { ker }{ }^{m} D_{(T, u)}\right)^{\perp},\left(\text { coker }{ }^{m} D_{(T, u)}\right)^{\perp}$ denote the $L^{2}$-orthogonal complement of the real line spanned by $i_{m}\left(\partial_{s} u^{m}\right)$ in ker $^{m} D_{(T, u)}$, coker ${ }^{m} D_{(T, u)}$, respectively. Then

$$
\left(\text { ker }^{m} D_{(T, u)}\right)^{\perp} \subset \text { ker }^{m} \mathcal{D}_{(T, u)},\left(\text { coker }^{m} D_{(T, u)}\right)^{\perp} \subset \text { coker }{ }^{m} \mathcal{D}_{(T, u)}
$$


and their $L^{2}$-orthogonal complements are at most one-dimensional and are invariant under the $\mathbb{Z} / m \mathbb{Z}$ action. Say $\gamma$ is a non-trivial element in either of these one-dimensional spaces, then its $\mathbb{R}$-component must be non-trivial. Observe however that the $\mathbb{R}$-component is invariant under the $\mathbb{Z} / m \mathbb{Z}$ action, so in fact $\mathbb{Z} / m \mathbb{Z}$ acts trivially on these orthogonal complements; they are therefore empty by our 1-non-degeneracy assumption. Hence

$$
\left(\text { ker }{ }^{m} D_{(T, u)}\right)^{\perp}=\operatorname{ker}{ }^{m} \mathcal{D}_{(T, u)},\left(\text { coker }{ }^{m} D_{(T, u)}\right)^{\perp}=\operatorname{coker}^{m} \mathcal{D}_{(T, u)},
$$

and we shall regard $\underline{\xi}_{i}, \underline{\eta}_{j}$ as elements in the kernel/cokernel of ${ }^{m} D_{(T, u)}$, respectively.

Claim. Let $J, X$ be as in the Lemma and let $H \in V_{\delta}^{k}(J, X)$ be such that $\mathcal{M}_{P}^{\leq 1, \Re}\left(J, X+\chi_{H}\right)$ and $\mathcal{M}_{O}^{\leq 1, \operatorname{sim}, \Re}\left(J, X+\chi_{H}\right)$ are $J$-regular. Let $(T, u) \in \mathcal{M}_{O}^{1, \operatorname{sim}, \Re}\left(J, X+\chi_{H}\right)$, and $m \in\{2,3, \ldots, n(u)\}$. Then there exists an arbitrarily small $h \in V_{\delta}^{k}(J, X)$ with the following properties:

(i) $\nabla h_{t}(u(\cdot, t))=0 \forall t$;

(ii) the function $h$ is supported on a small tubular neighborhood of the image of $u$ in $T_{f}$.

(iii) dim coker ${ }^{m} D_{(T, u)}^{J, X+\chi_{H+h}}<\operatorname{dim}$ coker ${ }^{m} D_{(T, u)}^{J, X+\chi_{H}}$ if $\operatorname{dim}$ coker ${ }^{m} D_{(T, u)}^{J, X+\chi_{H}} \neq 0$.

To finish the proof of the Lemma, apply the above Claim iteratively for each $u$ and $m$ until the dimension of all the relevant cokernels become 0 , requiring that the perturbation $h_{i}$ at step $i$ to be of $C_{\epsilon}$-norm smaller than $2^{-i} \varepsilon$, while also being small enough to preserve the non-degeneracy conditions already obtained. This can be done in finite steps since $\#\left(\hat{\mathcal{M}}_{O}^{0, \operatorname{sim}, \Re}\left(J, X+\chi_{H}\right)\right), n(u)$ and $p_{m}$ are all finite. Thus, we eventually obtain an $h=\sum_{i} h_{i}$ with $\|h\|_{C_{\epsilon}}<\varepsilon$ satisfying the desired properties.

Proof of the Claim. Suppose dimcoker ${ }^{m} D_{(T, u)}^{J, X+\chi_{H}} \neq 0$ and is non-decre asing under the perturbation by $h$. That means that for each $\underline{\eta}_{i}, i=$ $1, \ldots, p_{m}$, there is a small $\underline{\eta}_{0} \in L_{1}^{p}\left(V_{m}\right)$ such that

$$
\left({ }^{m} D_{(T, u)}^{J, X+\chi_{H+h}}\right)^{*}\left(\underline{\eta}_{i}+\underline{\eta}_{0}\right)=\left({ }^{m} D_{(T, u)}^{J, X+\chi_{H}}\right)^{*}\left(\underline{\eta}_{0}\right)+\nabla \nabla h(u)\left(\underline{\eta}_{i}+\underline{\eta}_{0}\right)=0 .
$$

Of course, this is possible only when

$$
\Pi_{\left(\operatorname{ker} m D_{(T, u)}^{J, X+\chi_{H}}\right)^{\perp}}\left(\nabla \nabla h(u)\left(\underline{\eta}_{i}+\underline{\eta}_{0}\right)\right)=0 .
$$

When $h$ is small, the set of such $h$ 's is modeled on the solution space of the equations:

$$
a_{i j}:=\left\langle\underline{\xi}_{j}, \nabla \nabla h(u) \underline{\eta}_{i}\right\rangle_{2}=0 \quad \forall i, j \in\left\{1,2, \ldots, p_{m}\right\} .
$$

We shall show that there exists some choice of $h$ such that the matrix $\left(a_{i j}\right)$ is non-trivial, contradicting (21). 
Since we shall choose $h$ to be supported on a small neighborhood, it suffices to consider restrictions of $\underline{\eta}_{j}, \underline{\xi}_{i}$ to a contractible subset of $\Theta_{u}$, over which $V_{m}$ splits as the direct sum of $m$ copies of $u^{*} K$. Let $\eta_{j}^{1}, \eta_{j}^{2}, \ldots, \eta_{j}^{m}$ be the components of $\underline{\eta}_{j}$ with respect to this splitting; similarly for $\xi_{i}^{k}, k=$ $1,2, \ldots, m$. Locally, $a_{i j}$ may be written as

$$
a_{i j}=\int \sum_{k} \operatorname{Tr}_{u^{*} K}\left(\nabla \nabla h(u) \eta_{j}^{k} \otimes \xi_{i}^{k}\right) d s d t .
$$

A direct computation from $(20)$ shows that the $\left(\Gamma\left(\operatorname{End}\left(u^{*} K\right)\right)\right.$-valued $)$ matrix

$$
\begin{aligned}
\left(A_{i j}\right) & :=\left(\sum_{k} \eta_{j}^{k} \otimes \xi_{i}^{k}\right) \\
& =\left(\sum_{k}\left(L^{k} \eta_{j}^{1}\right) \otimes\left(L^{k} \xi_{i}^{1}\right)\right) \\
& =F \oplus G,
\end{aligned}
$$

where

$$
\begin{aligned}
F & :=\left(\sum_{l, l^{\prime}=1}^{p^{\prime}} \frac{m \epsilon_{l l^{\prime}}}{2} \iota_{l l^{\prime}}\left(\begin{array}{cc}
\eta_{1}^{1} \otimes \xi_{1}^{1}+\eta_{2}^{1} \otimes \xi_{2}^{1} & \eta_{1}^{1} \otimes \xi_{2}^{1}-\eta_{2}^{1} \otimes \xi_{1}^{1} \\
-\eta_{1}^{1} \otimes \xi_{2}^{1}+\eta_{2}^{1} \otimes \xi_{1}^{1} & \eta_{1}^{1} \otimes \xi_{1}^{1}+\eta_{2}^{1} \otimes \xi_{2}^{1}
\end{array}\right)\right) ; \\
G & :=m\left(\eta_{j} \otimes \xi_{i}\right)_{p_{m} \geq i, j>2 p^{\prime}} .
\end{aligned}
$$

$\epsilon_{l l^{\prime}}=1$ when $q_{l}=q_{l^{\prime}} \bmod n$, and equals 0 otherwise; $\iota_{l l^{\prime}}$ is the embedding of the space of $2 \times 2$ matrices into the space of $\left(2 p^{\prime}\right) \times\left(2 p^{\prime}\right)$ matrices

$$
\iota_{l l^{\prime}}\left(\left(a_{p q}\right)\right)=\left(b_{i j}\right), \quad b_{i j}=a_{i-2 l+2, j-2 l^{\prime}+2},
$$

$a_{p q}=0$ if $p, q \notin\{1,2\}$.

Note that by the unique continuation theorem (cf. [14]), any element in $\operatorname{ker} D_{\left((m T), u^{m}\right)}^{J, X+\chi_{H}}$ or coker $D_{\left((m T), u^{m}\right)}^{J, X+\chi_{H}}$ are non-vanishing for all but isolated $(s, t)$, so we can find an $\left(s_{0}, t_{0}\right)$ away from $\Omega(u) \cup R(u)$ such that in a small neighborhood of which, $\xi_{1}^{1}, \eta_{1}^{1}$ are non-vanishing and not colinear with $\partial_{s} u$ (this is possible because $i_{m}^{-1} \underline{\xi}_{1}, i_{m}^{-1} \underline{\eta}_{1}$ are $L^{2}$-orthogonal to $\partial_{s} u^{m}$.

We shall choose $h$ to be of the following form. Let $\phi_{t}: U\left(u\left(s_{0}, t_{0}\right)\right) \rightarrow$ $\mathbb{R}^{2 n}=\left\{\left(\chi_{0}^{t}, \chi_{1}^{t}, \ldots, \chi_{2 n-1}^{t}\right) \mid \chi_{i}^{t} \in \mathbb{R}\right\}$ be $t$-dependent local coordinate charts on a neighborhood $U\left(u\left(s_{0}, t_{0}\right)\right)$ of $u\left(s_{0}, t_{0}\right) \in M$ such that $\phi_{t}(u(s, t))=$ $\left(s-s_{0}, 0, \ldots, 0\right)$, and $\phi_{t}$ is smooth in $t$. Let

$$
h_{t}(x)=\beta\left(t-t_{0}\right) \beta_{U\left(u\left(s_{0}, t_{0}\right)\right)}(x) \sum_{i, j=1}^{2 n-1} B^{i j} \chi_{i}^{t}(x) \chi_{j}^{t}(x),
$$

where $\beta: \mathbb{R} \rightarrow[0,1]$ is a smooth cutoff function supported in a small neighborhood near 0 , and $\beta_{U\left(u\left(s_{0}, t_{0}\right)\right)}$ is a smooth cutoff function on $M$ 
supported on $U\left(u\left(s_{0}, t_{0}\right)\right)$. $\left(B_{i j}\right)$ is a symmetric $(2 n-1) \times(2 n-1)$ matrix; we denote the corresponding bilinear form $B: \operatorname{Sym}^{2} T_{u\left(s_{0}, t_{0}\right)} M \rightarrow \mathbb{R}$.

This choice of $h$ apparently satisfies $h_{t}(u(s, t))=0 ; \nabla h_{t}(u(s, t))=0$. Moreover, since $\left(a_{i j}\right)=\left(\int \operatorname{Tr}_{u * K}\left(\nabla \nabla h A_{i j}\right) d s d t\right)$, if $A_{11}$ is in $G$, it is obviously possible to choose a $\left(B_{i j}\right)$ such that

$$
a_{11} \sim C B\left(\xi_{1}^{1}\left(s_{0}, t_{0}\right), \eta_{1}^{1}\left(s_{0}, t_{0}\right)\right) \neq 0
$$

for a constant $C$. If $A_{11}$ is in $F$ and $\eta_{2}^{1}, \xi_{2}^{1}$ are non-trivial multiples of $\eta_{1}^{1}, \xi_{1}^{1}$ or $\xi_{1}^{1}, \eta_{1}^{1}$ respectively, then by elementary algebra, for generic $B$ one of $a_{11}$ and $a_{12}$ must be non-vanishing, since they are of the form

$$
\begin{aligned}
& a_{11}=C\left(B\left(\xi_{1}^{1}\left(s_{0}, t_{0}\right), \eta_{1}^{1}\left(s_{0}, t_{0}\right)\right)+B\left(\xi_{2}^{1}\left(s_{0}, t_{0}\right), \eta_{2}^{1}\left(s_{0}, t_{0}\right)\right)\right)+o(1) \\
& a_{12}=C\left(B\left(\xi_{2}^{1}\left(s_{0}, t_{0}\right), \eta_{1}^{1}\left(s_{0}, t_{0}\right)\right)-B\left(\xi_{1}^{1}\left(s_{0}, t_{0}\right), \eta_{2}^{1}\left(s_{0}, t_{0}\right)\right)\right)+o(1) .
\end{aligned}
$$

In the remaining case, $B\left(\xi_{2}^{1}\left(s_{0}, t_{0}\right), \eta_{2}^{1}\left(s_{0}, t_{0}\right)\right)$ is either zero or can be chosen independent of $B\left(\xi_{1}^{1}\left(s_{0}, t_{0}\right), \eta_{1}^{1}\left(s_{0}, t_{0}\right)\right)$ so it is again easy to make $a_{11} \neq 0$.

\section{Main theorems from a general invariance theorem}

In this section, we derive the theorems stated in Section 2 from a general invariance theorem, Theorem 4.1.1 below. A rough outline of the proof for Theorem 4.1.1 is provided in Section 4.4, while the details are subjects of the remaining sections in this paper.

4.1. Proof of Theorem 2.3.3, assuming Theorem 4.1.1. Let $\Lambda \subset \mathbb{R}$ be an interval. A path $\left\{X_{\lambda}\right\}_{\lambda \in[1,2]}$ in $X$ is said to be $H^{1}$-codirectional if $\exists\langle y\rangle \in \operatorname{Hom}(\mathfrak{H} ; \mathbb{R})$ so that

$$
\left.\left\langle y_{X_{\lambda}}\right\rangle\right|_{\operatorname{ker} \psi}=\left.\alpha_{\lambda}\langle y\rangle\right|_{\operatorname{ker} \psi} \quad \text { for some } \alpha_{\lambda} \geq 0 \forall \lambda \text {. }
$$

4.1.1. Theorem. (General invariance theorem) Let $M$ be $w^{+}{ }_{-}$monotone, and $\left(J_{1}, X_{1}\right),\left(J_{2}, X_{2}\right)$ be two regular pairs. Suppose there exists an $H^{1}$-codirectional path $\left\{X_{\lambda}\right\}_{\lambda \in[1,2]}$ in $X$ connecting $X_{1}, X_{2}$. Then

$$
\begin{cases}I_{\mathrm{F}}\left(J_{1}, X_{1}\right)=I_{\mathrm{F}}\left(J_{2}, X_{2}\right) & \text { if } \alpha_{1}>0, \alpha_{2}>0, \text { or } \alpha_{1}=\alpha_{2}=0 \\ i_{-\langle y\rangle} I_{\mathrm{F}}\left(J_{1}, X_{1}\right)=I_{\mathrm{F}}\left(J_{2}, X_{2}\right) & \text { if } \alpha_{1}=0, \alpha_{2}>0 ; \\ i_{-\langle y\rangle} I_{\mathrm{F}}\left(J_{2}, X_{2}\right)=I_{\mathrm{F}}\left(J_{1}, X_{1}\right) & \text { if } \alpha_{2}=0, \alpha_{1}>0 .\end{cases}
$$

4.1.2. Proof of Theorem 2.3.3. Part (a): We show that the conditions of Theorem 2.3.3 imply the conditions of Theorem 4.1.1.

Let $X_{1}=X+\chi_{H_{1}}$ and $X_{2}=X+\chi_{H_{2}}$. Then they are connected by a path $\left\{X_{\lambda}\right\}$ in $\mathcal{X}$ of the form $X+\chi_{H_{\lambda}}$, since $\mathcal{H}$ is path-connected. The cohomology class $y_{X_{\lambda}}$ is independent of $\lambda$ in this case. Theorem 2.3.3(a) now follows immediately from Theorem 4.1.1. 
Part (b) is the consequence of the next, more general theorem.

4.1.3. Theorem. Let $(M, \omega)$ be $w^{+}$-monotone. If $M, f,\left[\gamma_{0}\right]$ satisfy either of the additional conditions (a) or (b) below, then $I_{\mathrm{F}}^{f,\left[\gamma_{0}\right]}(M)$ is invariant under symplectic isotopies in the sense of (8):

(a) There is an $\alpha \in \mathbb{R}$ such that

$$
\left.\omega\right|_{\pi_{2}(M)}=\left.\alpha c_{1}\right|_{\pi_{2}(M)} \text { as cohomology classes of } M .
$$

(b) $\left(f,\left[\gamma_{0}\right]\right)$ is "monotone" in the sense that $\psi_{\omega}^{f,\left[\gamma_{0}\right]}=\alpha \psi_{c}^{f,\left[\gamma_{0}\right]}$ for some $\alpha \in \mathbb{R}$.

In the last line, we added the superscripts $f,\left[\gamma_{0}\right]$ to the notation $\psi_{\omega}, \psi_{c}$ to emphasize their dependence on $f,\left[\gamma_{0}\right]$.

\section{Examples.}

(a) Condition (a) holds when $(M, \omega)$ is monotone.

(b) When $M$ is a Kähler-Einstein manifold, any $\left(f,\left[\gamma_{0}\right]\right)$ is monotone. A similar notion of "monotone symplectomorphisms" was introduced for the $n=1$ case in $[\mathbf{4 2}]$.

Proof of Theorem 4.1.3. Because $-\left\langle y_{X}\right\rangle=\psi_{\omega}^{f,\left[\gamma_{0}\right]}-\partial_{f}^{*}\left[\theta_{X}\right]$ and $X$ is pathconnected, if for fixed $f,\left[\gamma_{0}\right]$ there exists an $X_{0} \in X$ such that $\left.\left\langle y_{X_{0}}\right\rangle\right|_{\text {ker } \psi}=0$, any $X \in X$ can be connected to $X_{0}$ via a path satisfying (23). Invariance under symplectic isotopies would then follow from Theorem 4.1.1.

We now verify that this is true for both cases (a) and (b). In case (b), $\left.\psi_{\omega}\right|_{\text {ker } \psi}=\left.\alpha \psi_{c}\right|_{\text {ker } \psi_{c}}=0$, so $X_{0}$ can be simply taken to be 0 .

For case (a), note that an examination of the maps in (12) shows that there is a short exact sequence

$$
0 \rightarrow \mathfrak{H}^{\prime} \rightarrow \mathfrak{H} \rightarrow \mathfrak{H}^{\prime \prime} \rightarrow 0,
$$

where $\mathfrak{H}^{\prime}=$ Image $\left(\iota_{f *} \circ\right.$ Hurewicz $)$, and $\mathfrak{H}^{\prime \prime}=$ Image $\left(\partial_{f} \circ \mathrm{im}\right)$. On the other hand, (24) and the fact that $\left[c_{1}(T M)\right]=\iota_{f}^{*}\left[c^{f}\right],[\omega]=\iota_{f}^{*}\left[\omega_{f}\right]$ imply $\left.\left(\psi_{\omega}^{f,\left[\gamma_{0}\right]}-\alpha \psi_{c}^{f,\left[\gamma_{0}\right]}\right)\right|_{\mathfrak{H}^{\prime}}=0$. Thus, there exists a (non-unique) $\theta \in H^{1}(M)$ such that

$$
\psi_{\omega}^{f,\left[\gamma_{0}\right]}-\alpha \psi_{c}^{f,\left[\gamma_{0}\right]}=\left.\partial_{f}^{*} \theta\right|_{\mathfrak{H}} .
$$

Now choose $X_{0} \in \mathcal{X}$ such that its Calabi invariant $\left[\int \theta_{X_{0, t}} d t\right]=\theta$.

4.2. A fundamental example. We explain here how Corollary 2.3.4 follows from Theorem 2.3.3(b). It is restated in a slightly more general form as follows. 
4.2.1. Corollary. Let $(M, \omega)$ be $w^{+}$-monotone and supppose it satisfies (24). Let $\Phi=\left\{\phi_{\lambda} \mid \lambda \in[0,1]\right\}$ be a symplectic isotopy with $\phi_{0}=\mathrm{id}$, $\phi_{1}=f$, and $\gamma_{0}=\Phi \cdot \gamma_{p}, \gamma_{p}$ being the constant path at $p \in M$. Then $I_{\mathrm{F}}$ is invariant under symplectic isotopies in the sense of (8); furthermore, $I_{\mathrm{F}}^{f,\left[\gamma_{0}\right]}(M)=\tau(M)$.

Proof. In this case, the fibration (10) has a standard section, and therefore via the homotopy exact sequence of the fibration, we have the decomposition $\pi_{1}(\mathcal{C})=\pi_{1}(M) \times \pi_{2}(M)$. On the other hand, since in this case $T_{f} \simeq M \times S^{1}$, $H_{2}\left(T_{f} ; \mathbb{Z}\right)=H_{1}(M ; \mathbb{Z}) \oplus H_{2}(M ; \mathbb{Z})$, and im is simply the abelianization map. Thus,

$$
\mathfrak{H}=H_{1}(\mathcal{C} ; \mathbb{Z})=H_{1}(M ; \mathbb{Z}) \oplus \pi_{2}(M) .
$$

In terms of this decomposition,

$$
\begin{aligned}
\psi_{c} & =0 \oplus\left[c_{1}(T M)\right]: H_{1}(M) \oplus \pi_{2}(M) \rightarrow \mathbb{Z}, \\
\psi_{\omega} & =\theta_{\Phi} \oplus[\omega]: H_{1}(M) \oplus \pi_{2}(M) \rightarrow \mathbb{R},
\end{aligned}
$$

$\theta_{\Phi}$ being the Calabi invariant of $\Phi$. The coefficient ring for the Floer complex is

$$
\Lambda_{\mathrm{F}}=\operatorname{Nov}\left(H_{1}(M) \oplus \operatorname{Ker}\left(\left.\psi_{c}\right|_{\pi_{2}(M)}\right),-\theta \oplus[\omega] ; \mathbb{Z}\right) .
$$

By the monotonicity of $M$, Theorem 4.1.3(a) ensures the invariance under symplectic isotopies. Furthermore, by Section 3.1.4, we may assume without loss of generality that $f=\mathrm{id}$, and $\gamma_{0}=\gamma_{p}$.

Thus, $I_{\mathrm{F}}^{\mathrm{id},\left[\gamma_{p}\right]}(M)$ can be computed at a regular pair $(J, X)$ which is $t$-independent, with $X=\chi_{H}, H$ being a small Morse function on $M$. By a standard argument (see e.g., [12] and Section 7 of $[\mathbf{4 0}]$ ), such regular pairs exist, and $\mathcal{P}(X), \mathcal{M}_{P}(J, X), \mathcal{M}_{O}(J, X)$ in this case consist of $t$-independent elements, namely, critical points, gradient flow lines, and periodic orbits of $H$, respectively. Thus,

$$
\mathrm{CF}_{k \in \mathbb{Z} / 2 \mathbb{Z}}(J, X)=\mathrm{CM}_{k \in \mathbb{Z} / 2 \mathbb{Z}}(H) \otimes_{\mathbb{Z}\left[H_{1}(M ; \mathbb{Z})\right]} \mathbb{Z}\left[H_{1}(M ; \mathbb{Z}) \oplus \operatorname{Ker}\left(\left.\psi_{c}\right|_{\pi_{2}(M)}\right)\right],
$$

where $\mathrm{CM}_{*}(H)$ is the twisted Morse complex of $H$. Namely, it is defined in exactly the same way as the construction of CF in Section 2.2.2, modeling on the lift of the gradient flow of $H$ to the universal abelian covering $\tilde{M}$.

We also need to compare the orientations on the moduli spaces that enter into the definition of the boundary maps $\partial_{\mathrm{F}}$ and $\partial_{M}$. As mentioned in Remark 2.2.4, while on the Floer theory side the moduli spaces are endowed with coherent orientations (as in [13], and II.7.2 later), on the Morse theory side the moduli spaces are oriented via their interpretation as intersection spaces (as in e.g., [22]). Linearized versions of the adiabetic analysis which identified the Floer-theoretic moduli spaces with the Morse-theoretic moduli spaces also identify the Floer-theoretic coherent orientations with coherent orientations of solution spaces of the gradient flow equation of $H$. On the 
other hand, the arguments in [41] Section 3 and Appendix B shows that this analytic version of orientation agrees with the geometric version of orientation. Thus, the complexes $\mathrm{CF}_{*}(J, X)$ and $\mathrm{CM}_{*}(H)$ have the same boundary map $\partial_{\mathrm{F}}=\partial_{M}$.

Lastly, $\mathcal{M}_{O}\left(J, \chi_{H}\right)=\emptyset$ since the gradient flow of the real-valued function $H$ has no periodic orbits. Thus,

$$
I_{\mathrm{F}}^{\mathrm{id},\left[\gamma_{p}\right]}(M)=\iota_{*} \tau(\mathrm{CM}(H))=\iota_{*} \tau(M) .
$$

The second equality above is the well-known equivalence between torsions of Morse complexes (of real-valued Morse functions) and torsions of cell chain complexes (see e.g., [35]).

Remark. In [32], Le and Ono considered a Floer theory under assumptions similar to Theorem 4.1.3. There they showed that in this case, a (twisted version of) Floer homology is equivalent, up to a change of coefficients, to a Novikov homology (a version of twisted Morse homology).

Their definition of Floer complex is different from ours in that they used a smaller covering of the loop space. The advantage of this choice is that the associated homology retains more information (i.e., is less likely to be trivial), though it has worse invariance properties. In this paper, our main concern is torsion, and the torsions associated to different coverings are related by a simple change of coefficients induced by maps between covering groups. For our purpose the larger covering is therefore the better choice.

4.3. Regular homotopy of Floer systems. The proof of Theorem 4.1.1 will rely on the existence of a certain path of formal vector fields $\left\{\mathcal{V}_{\lambda}\right\}$ connecting the two generated by $\left(J_{1}, X_{1}\right)$ and $\left(J_{2}, X_{2}\right)$. The purpose of this subsection is to describe such paths of vector fields, called "regular homotopy of Floer systems".

We shall work on an abstract level for the rest of this section, as we did in Section 2.1. The eventual goal is to give a formal proof of the invariance of $I_{\mathrm{F}}$ (cf. Proposition 4.4.6), which applies to any Floer theory satisfying the outline in Section 2.1.

4.3.1. CHFSs. A codirectional homotopy of Floer systems (CHFS for short) is a 1-parameter family of formal flows $\left\{\left(\mathcal{C}, \mathfrak{H}, \text { ind; } y_{\lambda}, \mathcal{V}_{\lambda}\right)\right\}_{\lambda \in \Lambda=[1,2]}$ (abbreviated as $\left\{\mathcal{V}_{\lambda}\right\}_{\lambda \in \Lambda=[1,2]}$ ) such that:

- $\mathcal{V}_{1}, \mathcal{V}_{2}$ generate Floer systems with the same SF-homomorphism $\psi$, and

- $\exists \lambda$-independent $\langle y\rangle \in \operatorname{Hom}(\mathfrak{H}, \mathbb{R})$, so that $\left.\left\langle y_{\lambda}\right\rangle\right|_{\operatorname{ker} \psi}=\left.\alpha_{\lambda}\langle y\rangle\right|_{\operatorname{ker} \psi}$ for some $\alpha_{\lambda} \geq 0 \forall \lambda$.

Such a $\langle y\rangle$ will be called a c-class of the CHFS. Notice that for a CHFS, the associated Novikov ring $\operatorname{Nov}\left(\operatorname{ker} \psi,-\left\langle y_{\lambda}\right\rangle ; \mathbb{Z}\right)=\operatorname{Nov}(\operatorname{ker} \psi,-\langle y\rangle ; \mathbb{Z})$ remains the same through the homotopy. 
Given a closed interval $S \subset \Lambda$, we say that $\left\{\mathcal{V}_{\lambda}\right\}_{\lambda \in S}$ is a sub-homotopy over $S$ of the CHFS $\left\{\mathcal{V}_{\lambda}\right\}_{\lambda \in \Lambda}$.

Define the parametrized moduli spaces $\mathcal{P}^{\Lambda}=\bigcup_{\lambda} \mathcal{P}_{\lambda}, \mathcal{M}_{P}^{\Lambda, k+1}=\bigcup_{\lambda} \mathcal{M}_{P, \lambda}^{k}$, $\mathcal{M}_{O}^{\Lambda, k+1}=\bigcup_{\lambda} \mathcal{M}_{O, \lambda}^{k}$, where $\mathcal{P}_{\lambda}, \mathcal{M}_{P, \lambda}, \mathcal{M}_{O, \lambda}$ are moduli spaces of the formal flow generated by $\mathcal{V}_{\lambda}$. We denote the projection of these parametrized moduli spaces to $\Lambda$ by $\Pi_{\Lambda}$ in general. In addition, define

$$
\mathcal{P}^{\Lambda, \operatorname{deg}}=\mathcal{P}^{\Lambda} \backslash \bigcup_{\lambda \in \Lambda} \mathcal{P}_{\lambda, \mathrm{ndg}} .
$$

A CHFS is regular (i.e., is an RHFS) if the parametrized moduli spaces satisfy all the properties (RHFS*) below. These properties are written modeling on the generic behavior of a 1-parameter family of flows in finitedimensional Morse-Novikov theory.

\subsubsection{Properties of $\mathcal{P} \Lambda, \mathcal{P} \Lambda, \operatorname{deg}$.}

(RHFS1) $\mathcal{P}^{\Lambda}$ is a compact Zariski smooth 1-manifold with boundary $\partial \mathcal{P}^{\Lambda}=$ $\mathcal{P}_{2}-\mathcal{P}_{1}$. The space $\mathcal{P}^{\Lambda, \operatorname{deg}}$ coincides with the set of critical points of $\Pi_{\Lambda}$; in addition, it consists of finitely many points in the interior of $\mathcal{P}^{\Lambda}$, which are extrema of $\Pi_{\Lambda}$.

In fact, the identifcation of $\mathcal{P}^{\Lambda, \operatorname{deg}}$ with critical points of $\Pi_{\Lambda}$ is a direct consequence of the Zariski smoothness of $\mathcal{P} \Lambda$. To see this, note that the deformation operator for $\mathcal{P}^{\Lambda}$ at $x_{\lambda}$, denoted $\hat{A}_{x_{\lambda}}$, is a rank 1 extension of $A_{x_{\lambda}}$ given by

$$
\hat{A}_{x_{\lambda}}(\alpha, \xi)=\alpha \partial_{\lambda} \mathcal{V}_{\lambda}+A_{x_{\lambda}} \xi \quad \text { for } \alpha \in \mathbb{R}, \xi \in T_{x_{\lambda}} C .
$$

Apparently, $\operatorname{ker} A_{x_{\lambda}} \subset \operatorname{ker} \hat{A}_{x_{\lambda}}$. Zariski smoothess of $\mathcal{P} \Lambda$ implies that the latter is one-dimensional, and therefore, the former is either zero-dimensional or one-dimensional. In the first case, $x_{\lambda}$ is a non-degenerate element of $\mathcal{P}_{\lambda}$; in the second case, it is said to be minimally degenerate. Furthermore, notice that $d \Pi_{\Lambda}: T_{x_{\lambda}} \mathcal{P} \Lambda \rightarrow T_{\lambda} \Lambda$ is given by projecting ker $\hat{A}_{x_{\lambda}} \subset \mathbb{R} \oplus T_{x_{\lambda}} \mathrm{e}$ to the $\mathbb{R}$-summand. This projection is trivial precisely when $x_{\lambda}$ is minimally degenerate, and hence $\mathcal{P}^{\Lambda, \text { deg }}$ consists precisely of critical points of the $\operatorname{map} \Pi_{\Lambda}$.

(RHFS1i) (Injectivity) The restriction $\left.\Pi_{\Lambda}\right|_{\mathcal{P}^{\Lambda, \mathrm{deg}}}$ is injective.

Elements of $\mathcal{P}^{\Lambda, \operatorname{deg}}$ are called death-births: a local maximum of $\Pi_{\Lambda}$ is called a death, and a local minimum is called a birth. We denote

$$
\Lambda_{\mathrm{db}}:=\Pi_{\Lambda}\left(\mathcal{P}^{\Lambda, \operatorname{deg}}\right) \subset \Lambda .
$$

A small neighborhood $S$ of a subset $P \subset \Lambda_{\mathrm{db}}$ is called a death-birth neighborhood. The half $\Pi^{\Lambda}\left(\mathcal{P}^{S} \backslash \mathcal{P}^{S \text {,deg }}\right) \subset S$ is called a birth-neighborhood, and the other half $S \backslash \Pi^{\Lambda}\left(\mathcal{P}^{S}\right)$ is called a death-neighborhood. 
Let $\tilde{\mathcal{P}}^{\Lambda}, \tilde{\mathcal{P}}^{\Lambda, \text { deg }}$ be, respectively, the lifts of $\mathcal{P} \Lambda, \mathcal{P} \Lambda$,deg $\subset \Lambda \times \mathcal{C}$ to $\Lambda \times \tilde{\mathcal{C}}$.

Note that ind (respectively gr) defines a locally constant function on $\mathcal{P}^{\Lambda} \backslash \mathcal{P}^{\Lambda, \text { deg }}$ (respectively $\left.\left(\tilde{\mathcal{P}}^{\Lambda} \backslash \tilde{\mathcal{P}}^{\Lambda, \text { deg }}\right)^{\times 2}\right)$. Let $\operatorname{gr}_{+}$, gr gr $_{-}\left(\tilde{\mathcal{P}}^{\Lambda}\right)^{\times 2} \rightarrow \mathbb{Z}$ denote the upper-semicontinuous and the lower-semicontinuous extension of gr, respectively. Similarly for ind $_{+}$, ind in $_{-} \Lambda \rightarrow \mathbb{Z} / 2 \mathbb{Z}$. It follows from the definition of ind, gr via spectral flow and the fact that $\operatorname{ker} A_{x_{\lambda}}=1$ for any $x_{\lambda} \in \mathcal{P}^{\Lambda, \operatorname{deg}}$ that:

$$
\begin{aligned}
\operatorname{gr}_{-}\left(\left(x_{\lambda},\left[w_{\lambda}\right]\right), \cdot\right) & =\operatorname{gr}_{+}\left(\cdot,\left(x_{\lambda},\left[w_{\lambda}\right]\right)\right)-1 \\
\operatorname{gr}_{-}\left(\cdot,\left(x_{\lambda},\left[w_{\lambda}\right]\right)\right) & =\operatorname{gr}_{+}\left(\left(x_{\lambda},\left[w_{\lambda}\right]\right), \cdot\right)-1 \\
\operatorname{ind}_{-}\left(x_{\lambda}\right) & =\operatorname{ind}_{+}\left(x_{\lambda}\right)-1 \text { for any }\left(x_{\lambda},\left[w_{\lambda}\right]\right) \in \tilde{\mathcal{P}}^{\Lambda, \operatorname{deg}} .
\end{aligned}
$$

By (RHFS1, 1i), $\mathcal{P}^{\Lambda} \backslash \mathcal{P}^{\Lambda \text {,deg }}$ consists of finitely many path components, and $\Pi_{\Lambda}$ restricts to a diffeomorphism from each such component $\mathbf{x}$ to its image in $\Lambda$, which we denote by $\Lambda_{\mathbf{x}}$. Denote the set of such path components by $\aleph_{\Lambda}$. Similarly, let $\tilde{\aleph}_{\Lambda}$ be the set of path components of $\tilde{\mathcal{P}}^{\Lambda} \backslash \tilde{\mathcal{P}}^{\Lambda, \text { deg }}$. Given $\lambda \in \Lambda$, we denote by $x_{\lambda}$

$$
x_{\lambda}=\Pi_{\Lambda}^{-1}(\lambda) \cap \mathbf{x} .
$$

Since ind is constant over $\mathbf{x}$, we denote $\operatorname{ind}(\mathbf{x})=\operatorname{ind}\left(x_{\lambda}\right)$ for an arbitrary $\lambda \in \Lambda_{\mathbf{x}}$. Similarly for gr.

4.3.3. Weight truncation associated to $\langle y\rangle$ and $e_{\mathcal{P}}$. Before proceeding to the next property of RHFS, we shall describe a truncation of the moduli spaces by certain weight determined by $\langle y\rangle$. This is essentially equivalent to truncating by energy, but is better suited for a uniform description of the moduli spaces in a CHFS.

Given a formal flow $\left(\mathcal{C}, \mathfrak{H}\right.$, ind; $\left.\mathfrak{y}_{\chi}, \mathcal{V}_{\chi}\right)$, choose a $Y \in \operatorname{Hom}(\mathfrak{H}, \mathbb{R})$ and an basis $e_{\mathcal{P}}$ for $\mathrm{CF}$ as in Section 2.2.3 (2.3). Namely, a lifting Lf $: \mathcal{P} \rightarrow \tilde{\mathcal{P}}$, and a choice of $A_{0} \in \mathfrak{H}$ such that $\psi\left(A_{0}\right)=2 \mathbb{N}_{\psi}$ when $\mathbb{N}_{\psi} \neq 0$. In this case, this choice decides a splitting

$$
\mathfrak{H}=\operatorname{ker} \psi \oplus \mathbb{Z} A_{0}
$$

A connecting flow line $u$ belongs to certain $\mathcal{M}_{P}(\operatorname{Lf}(x), A \cdot \operatorname{Lf}(y))$ for a unique $A \in \mathfrak{H}$, and $[u]_{\mathfrak{H}}:=A$ is said to be the $\mathfrak{H}$-class of $u$. On the other hand, when $u$ is a closed orbit, then $[u]_{\mathfrak{H}}:=A$ when $u \in \mathcal{M}_{O}(A)$. The ker $\psi$-class of $u$, denoted $[u]_{\operatorname{ker} \psi}$, is the projection of $[u]_{\mathfrak{H}}$ to $\operatorname{ker} \psi$ according to the above splitting determined by $A_{0}$; in the case when $\mathbb{N}_{\psi}=0$, $[u]_{\operatorname{ker} \psi}:=[u]_{\mathfrak{H}}$. Similarly, for $A \in \mathfrak{H},[A]_{\operatorname{ker} \psi}$ denotes its projection to ker $\psi$, in accordance with the above splitting when $\mathbb{N}_{\psi} \neq 0$ (otherwise ker $\psi=\mathfrak{H}$, and $\left.[A]_{\text {ker } \psi}=A\right)$. The weight of a $u \in \mathcal{M}_{P}$ or $\mathcal{M}_{O}$, denoted $\mathrm{wt}_{-Y, e_{\mathcal{P}}}(u)$, is defined to be $-Y[u]_{\operatorname{ker} \psi}$. 
For a CHFS, we define the weight for elements in $\mathcal{M}_{P}^{\Lambda}$ or $\mathcal{M}_{O}^{\Lambda}$ by fixing a lifting $\mathcal{P}^{\Lambda} \rightarrow \tilde{\mathcal{P}}^{\Lambda}$ (which in turn gives a consistent system of liftings Lf $: \mathcal{P}_{\lambda} \rightarrow \tilde{\mathcal{P}}_{\lambda}$ for each $\lambda \in \Lambda$ ), fixing a $\lambda$-independent $A_{0}$, and letting $Y=\langle y\rangle \forall \lambda$ for a c-class $\langle y\rangle$. Such a weight is said to be adapted to the $\operatorname{CHFS}\left\{\mathcal{V}_{\lambda}\right\}$.

Given $\Re \in \mathbb{R}$, let

$$
\mathcal{M}_{P}^{S, k}\left(\mathbf{x}, \mathbf{y} ; \mathrm{wt}_{-\langle y\rangle, e_{\mathcal{P}}} \leq \Re\right), \quad \hat{\mathcal{M}}_{P, \lambda}^{k}\left(x_{\lambda}, y_{\lambda} ; \mathrm{wt}_{-\langle y\rangle, e_{\mathcal{P}}} \leq \Re\right)
$$

be the moduli spaces consisting of flows with weight $\leq \Re$ and appropriate additional constraints. Similarly, for other variants of moduli spaces and $\mathcal{M}_{O}, \mathcal{M}_{O}^{\Lambda}$.

Let $\tilde{\mathcal{A}}_{\lambda}$ be the primitive of $y_{\lambda}$ with $\tilde{\mathcal{A}}_{\lambda}\left(\gamma_{0}\right)=0$. Then

$$
\mathcal{E}\left(u_{\lambda}\right)= \begin{cases}-\left\langle y_{\lambda}\right\rangle\left[u_{\lambda}\right]_{\mathfrak{H}}-\tilde{\mathcal{A}}_{\lambda}\left(\operatorname{Lf}\left(y_{\lambda}\right)\right)+\tilde{\mathcal{A}}_{\lambda}\left(\operatorname{Lf}\left(x_{\lambda}\right)\right) & \text { if } u_{\lambda} \in \mathcal{M}_{P, \lambda}\left(x_{\lambda}, y_{\lambda}\right) ; \\ -\left\langle y_{\lambda}\right\rangle\left[u_{\lambda}\right]_{\mathfrak{H}} & \text { if } u_{\lambda} \in \mathcal{M}_{\lambda, O} .\end{cases}
$$

Thus, we have the following relation between energy and weight:

$$
\mathcal{E}\left(u_{\lambda}\right)=\left\{\begin{array}{cc}
\alpha_{\lambda} \mathrm{wt} & \\
-\langle y\rangle, e_{\mathcal{P}} & \left(u_{\lambda}\right)+\beta_{\lambda} \psi\left(\left[u_{\lambda}\right]_{\mathfrak{H}}\right)+\tilde{\mathcal{A}}_{\lambda}\left(\operatorname{Lf}\left(x_{\lambda}\right)\right)-\tilde{\mathcal{A}}_{\lambda}\left(\operatorname{Lf}\left(y_{\lambda}\right)\right) \\
\alpha_{\lambda} \mathrm{wt} & \text { if } u_{\lambda} \in \mathcal{M}_{P, \lambda}\left(x_{\lambda}, y_{\lambda}\right)
\end{array}\right.
$$

where $\beta_{\lambda}=-\left\langle y_{\lambda}\right\rangle\left(A_{0}\right) /\left(2 \mathbb{N}_{\psi}\right)$ is continuous in $\lambda$.

For elements in $\mathcal{M}_{P, \lambda}^{k}$ or $\mathcal{M}_{O}^{k}$, all terms above are fixed except for the first term involving the weight. Thus, the filtration by weights on these moduli spaces are equivalent to filtration by energy when $\alpha_{\lambda}>0$, while when $\alpha_{\lambda}=0$, the energy filtration is the trivial filtration. Furthermore, as the constant terms above vary continuously in $\lambda$, and $\mathcal{P} \Lambda$ is compact, the above formula gives a uniform bound on energy by weight.

4.3.4. Structure of $\mathcal{M}_{P, \lambda}$ for $\lambda \in \Lambda_{\mathrm{db}}$. In the case when $x, y \in \mathcal{P}$ are either non-degenerate or minimally-degenerate, let $\mathcal{M}_{P}^{k}(x, y)$ be the moduli space of connecting flow lines which lift to be paths from $(x,[w])$ to $(y,[v])$ in $\tilde{\mathrm{C}}$, with $\operatorname{gr}_{+}((x,[w]),(y,[v])=k$.

(RHFS2d) (moduli at Death-births) For any $\lambda \in \Lambda_{\mathrm{db}}$, any integer $k<2$, and any pair $x_{\lambda}, y_{\lambda} \in \mathcal{P}_{\lambda}, \mathcal{M}_{P, \lambda}\left(\left(x_{\lambda},\left[w_{\lambda}\right]\right),\left(y_{\lambda},\left[v_{\lambda}\right]\right)\right)$ is a Zariski smooth manifold of dimension $k$. Furthermore, for any real constant $\Re$, the moduli space $\hat{\mathcal{M}}_{P}^{0}\left(x_{\lambda}, y_{\lambda} ; \mathrm{wt}_{-\langle y\rangle, e_{\mathcal{P}}} \leq \Re\right)$ consists of finitely many Zariski smooth points.

When $x_{\lambda}, y_{\lambda}$ are both non-degenerate, the above statement is part of property (FS2). 
4.3.5. The Structure of $\mathcal{M}_{P}^{\Lambda}$. Consider the parameterized moduli spaces over an interval $S \subset \Lambda$ :

$$
\begin{aligned}
\hat{\mathcal{M}}_{P}^{S}((\mathbf{x},[\mathbf{w}]),(\mathbf{y},[\mathbf{v}])) & :=\bigcup_{\lambda \in S \cap \Lambda_{\mathbf{x}} \cap \Lambda_{\mathbf{y}}} \hat{\mathcal{M}}_{P, \lambda}\left(\left(x_{\lambda},\left[w_{\lambda}\right]\right),\left(y_{\lambda},\left[v_{\lambda}\right]\right)\right), \\
\hat{\mathcal{M}}_{O}^{S} & :=\bigcup_{\lambda \in S} \hat{\mathcal{M}}_{O, \lambda} .
\end{aligned}
$$

These are included in parameterized versions of moduli spaces of broken trajectories/orbits,

$$
\begin{aligned}
\hat{\mathcal{M}}_{P}^{S,+}(\underline{(\mathbf{x},[\mathbf{w}]}),(\underline{\mathbf{y},[\mathbf{v}])}): & :=\bigcup_{\lambda \in \bar{S} \cap \bar{\Lambda}_{\mathbf{x}} \cap \bar{\Lambda}_{\mathbf{y}}} \hat{\mathcal{M}}_{P, \lambda}^{+}(\underline{(\mathbf{x},[\mathbf{w}]}),(\underline{\mathbf{y},[\mathbf{v}])}), \\
\hat{\mathcal{M}}_{O}^{S,+} & :=\bigcup_{\lambda \in \bar{S}} \hat{\mathcal{M}}_{O, \lambda}^{+},
\end{aligned}
$$

where $\hat{\mathcal{M}}_{P, \lambda}^{+}((\underline{\mathbf{x},[\mathbf{w}]}),(\underline{\mathbf{y},[\mathbf{v}]}))$ is the reduced, broken-trajectories variant of:

$$
\mathcal{M}_{P, \lambda}((\underline{\mathbf{x},[\mathbf{w}]}),(\underline{\mathbf{y},[\mathbf{v}]})):=\mathcal{M}_{P, \lambda}^{\left(\sigma_{1}, \sigma_{2}\right)}\left(\left(x_{\lambda},\left[w_{\lambda}\right]\right),\left(y_{\lambda},\left[v_{\lambda}\right]\right)\right),
$$

with weights $\sigma_{1}, \sigma_{2}$ chosen such that

$$
\operatorname{gr}^{\left(\sigma_{1}, 0\right)}\left(\left(x_{\lambda},\left[w_{\lambda}\right]\right), \cdot\right)=\operatorname{gr}((\mathbf{x},[\mathbf{w}]), \cdot) ; \quad \operatorname{gr}^{\left(\sigma_{2}, 0\right)}\left(\left(y_{\lambda},\left[v_{\lambda}\right]\right), \cdot\right)=\operatorname{gr}((\mathbf{y},[\mathbf{v}]), \cdot) .
$$

Other variants of the moduli spaces such as $\hat{\mathcal{M}}_{P}^{S, k,+}\left(\underline{\mathbf{x}}, \underline{\mathbf{y}}\right.$; wt $\left.\mathrm{w}_{-\langle y\rangle, \text { e्⿱ }} \leq \Re\right)$ can be defined in a similar way. The definition of chain topology extends to these parameterized versions in an obvious manner.

(RHFS2) Given $\mathbf{x}, \mathbf{y} \in \aleph_{\Lambda}$, an integer $k \leq 1$, and an interval $S \subset \Lambda$, the parameterized moduli space $\hat{\mathcal{M}}_{P}^{S, k}(\mathbf{x}, \mathbf{y})$ is a Zariski smooth-manifold of dimension $\operatorname{gr}(\mathbf{x}, \mathbf{y})$; furthermore, the parameterized moduli space of broken trajectories $\hat{\mathcal{M}}_{P}^{\Lambda, k,+}\left(\underline{\mathbf{x}}, \underline{\mathbf{y}}\right.$; wt $\left.-\langle y\rangle, e_{\mathcal{P}} \leq \Re\right)$ is compact for any $\Re$.

Notice that since $\hat{\mathcal{M}}_{P}^{S, 0,+}\left(\underline{\mathbf{x}}, \underline{\mathbf{y}} ; \mathrm{wt}_{-\langle y\rangle, e_{\mathcal{P}}} \leq \Re\right)=\hat{\mathcal{M}}_{P}^{S, 0}\left(\mathbf{x}, \mathbf{y} ; \mathrm{wt}_{-\langle y\rangle, e_{\mathcal{P}}} \leq\right.$ $\Re)$, (RHFS2) implies that this moduli space consists of finitely many non-degenerate points.

(RHFS2i) (Injectivity) The projection $\Pi_{\Lambda}$ is injective on the space

$$
\hat{\mathcal{M}}_{P}^{S, 0}:=\coprod_{\mathbf{x}, \mathbf{y} \in \aleph_{\Lambda}} \hat{\mathcal{M}}_{P}^{S, 0}(\mathbf{x}, \mathbf{y}) .
$$


Elements in this space are called handle-slides. They are said to be of type $I I$ if $\mathbf{x}=\mathbf{y}$. Otherwise they are of type $I$. Let

$$
\begin{aligned}
\Lambda_{h s}^{\Re} & :=\Pi_{\Lambda}\left(\coprod_{\mathbf{x}, \mathbf{y} \in \aleph_{\Lambda}} \hat{\mathcal{M}}_{P}^{\Lambda, 0}\left(\mathbf{x}, \mathbf{y} ; \mathrm{wt}_{-\langle y\rangle, e_{\mathcal{P}}} \leq \Re\right)\right) ; \\
\Lambda_{h s: i i}^{\Re} & :=\Pi_{\Lambda}\left(\coprod_{\mathbf{x} \in \aleph_{\Lambda}} \hat{\mathcal{M}}_{P}^{\Lambda, 0}\left(\mathbf{x}, \mathbf{x} ; \mathrm{wt}_{-\langle y\rangle, e_{\mathcal{P}}} \leq \Re\right)\right) ; \\
\Lambda_{h s} & :=\bigcup_{\Re} \Lambda_{h s}^{\Re} ; \quad \Lambda_{h s: i i}:=\bigcup_{\Re} \Lambda_{h s: i i}^{\Re} .
\end{aligned}
$$

These subsets of $\Lambda$ are disjoint from $\Lambda_{\mathrm{db}}$.

To state the next property, introduce the following zero-dimensional stratum in $\hat{\mathcal{M}}_{P}^{\Lambda, 1,+}\left(\underline{\mathbf{x}}, \mathbf{y} ; \mathrm{wt}_{-\langle y\rangle, e_{\mathrm{p}}} \leq \Re\right)$ : let $\mathbb{T}_{P, \mathrm{hs}-\mathrm{m}}(\mathbf{x}, \mathbf{y} ; \Re)$ be the subset consisting of $k$-th broken trajectories connected at non-degenerate critical points, with $k>1$. (RHFS2i) implies that such broken trajectories occur (only) with Type II handle-slides (namely, $\Pi_{\Lambda} \mathbb{T}_{P, \mathrm{hs}-\mathrm{m}}(\mathbf{x}, \mathbf{y} ; \Re)$ surjects to $\left.\Lambda_{h s: i i}^{\Re}\right)$.

Let $\mathbb{I}_{P}(\mathbf{x}, \mathbf{y} ; \Re):=\hat{\mathcal{M}}_{P}^{\Lambda, 1,+}\left(\underline{\mathbf{x}}, \underline{\mathbf{y}} ; \mathrm{wt}_{-\langle y\rangle, e_{\mathcal{P}}} \leq \Re\right) \backslash \hat{\mathcal{M}}_{P}^{\Lambda, 1}\left(\mathbf{x}, \mathbf{y} ; \mathrm{wt}_{-\langle y\rangle, e_{\mathcal{P}}} \leq \Re\right)$.

(RHFS2c) (Corner structure) The moduli space of broken trajectories, $\hat{\mathcal{M}}_{P}^{\Lambda, 1,+}\left(\underline{\mathbf{x}}, \underline{\mathbf{y}} ; \mathrm{wt}_{-\langle y\rangle, e_{\mathcal{P}}} \leq \Re\right)$, is lmb along $\mathbb{I}_{P}(\mathbf{x}, \mathbf{y} ; \Re) \backslash \mathbb{T}_{P, \mathrm{hs}-\mathrm{m}}(\mathbf{x}, \mathbf{y} ; \Re)$.

\subsubsection{The Structure of $\mathcal{M}_{O}^{\Lambda}$.}

(RHFS3) Given $k \leq 1$, the parameterized moduli space $\mathcal{M}_{O}^{S, k+1}(A)$ is a Zariski smooth-manifold of dimension $k+1$ with a semi-free $S^{1}$ action. Furthermore, The space $\hat{\mathcal{M}}_{O}^{S, k,+}\left(\mathrm{wt}_{-\langle y\rangle} \leq \Re\right)$ is compact.

(RHFS3c) (Corner structure) The moduli space of broken orbits, $\left.\hat{\mathcal{M}}_{O}^{\Lambda, 1,+}\left(\mathrm{wt}_{-\langle y\rangle, \text { ep }} \leq \Re\right)\right)$, is lmb along $\mathbb{I}_{O}(\Re) \backslash \mathbb{T}_{O, \mathrm{hs}-\mathrm{m}}(\Re)$, where $\mathbb{I}_{O}(\Re)$ and $\mathbb{T}_{O, \mathrm{hs}-\mathrm{m}}(\Re)$ are defined similarly to $\mathbb{I}_{P}(\mathbf{x}, \mathbf{y} ; \Re), \mathbb{T}_{P, \mathrm{hs}-\mathrm{m}}(\mathbf{x}, \mathbf{y} ; \Re)$.

4.3.7. Orientations. Let $\operatorname{sign}(p: P)$ denote the sign of $p \in \partial P$ oriented as a boundary point of the oriented 1-manifold $P$, and the sign of an $x_{\lambda} \in \mathcal{P} \Lambda$,deg be defined by

$$
\operatorname{sign}\left(x_{\lambda}\right)= \begin{cases}+1 & \text { when } x_{\lambda} \text { is a death } \\ -1 & \text { when } x_{\lambda} \text { is a birth }\end{cases}
$$

Notice that that $\operatorname{sign}\left(x_{\lambda}\right)=\operatorname{sign}\left(\lambda: \Lambda_{\mathbf{x}}\right)$ when $x_{\lambda} \in \mathcal{P}^{\Lambda, \text { deg }}$ are on the boundary of the path component $\mathbf{x} \subset \mathcal{P} \backslash \mathcal{P}^{\Lambda, \operatorname{deg}}$.

The notions of coherent orientation and grading-compatible orientation can be extended to parameterized moduli spaces, as will be explained in II.7.2. 
(RHFS4) The parameterized moduli spaces of connecting flow lines $\left\{\hat{\mathcal{M}}_{P}^{S, k}(\mathbf{x}, \mathbf{y})\right\}$ can be endowed with a coherent orientation, and the parameterized moduli space of closed orbits $\mathcal{M}_{O}^{S, 2}$ can be endowed with a gradingcompatible orientation. With respect to these orientations, the strata

$$
\mathbb{I}_{P}(\mathbf{x}, \mathbf{y} ; \Re) \backslash \mathbb{T}_{P, \mathrm{hs}-\mathrm{m}}(\mathbf{x}, \mathbf{y} ; \Re) \text { and } \mathbb{I}_{O}(\Re) \backslash \mathbb{T}_{O, \mathrm{hs}-\mathrm{m}}(\Re),
$$

oriented, respectively, as boundary components of the parameterized moduli spaces

$$
\hat{\mathcal{M}}_{P}^{\Lambda, 1,+}\left(\underline{\mathbf{x}}, \underline{\mathbf{y}} ; \mathrm{wt}_{-\langle y\rangle, e_{\mathcal{P}}} \leq \Re\right) \text { and } \hat{\mathcal{M}}_{O}^{\Lambda, 1,+}\left(\mathrm{wt}_{-\langle y\rangle, e_{\mathcal{P}}} \leq \Re\right)
$$

are expressed in terms of lower-dimensional moduli spaces as follows:

$$
\begin{aligned}
\mathbb{I}_{P}(\mathbf{x}, \mathbf{y} ; \Re) \backslash \mathbb{T}_{P, \mathrm{hs}-\mathrm{m}}(\mathbf{x}, \mathbf{y} ; \Re)=\mathbb{J}_{P}(\mathbf{x}, \mathbf{y} ; \Re) \sqcup \mathbb{T}_{P, \mathrm{db}}(\mathbf{x}, \mathbf{y} ; \Re) \sqcup \mathbb{T}_{P, \mathrm{hs}-\mathrm{s}}(\mathbf{x}, \mathbf{y} ; \Re) ; \\
\mathbb{I}_{O}(\Re) \backslash \mathbb{T}_{O, \mathrm{hs}-\mathrm{m}}(\Re)=\mathbb{J}_{O}(\Re) \sqcup \mathbb{T}_{O, \mathrm{db}}(\Re) \sqcup \mathbb{T}_{O, \mathrm{hs}-\mathrm{s}}(\Re),
\end{aligned}
$$

where

$$
\begin{aligned}
& \mathbb{J}_{P}(S, \mathbf{x}, \mathbf{y} ; \Re)= \\
& \quad \coprod_{\lambda \in \partial\left(S \cap \Lambda_{\mathbf{x}} \cap \Lambda_{\mathbf{y}}\right)} \operatorname{sign}\left(\lambda: S \cap \Lambda_{\mathbf{x}} \cap \Lambda_{\mathbf{y}}\right) \hat{\mathcal{M}}_{P, \lambda}^{0}\left(\underline{\mathbf{x}}, \underline{\mathbf{y}} ; \mathrm{wt}_{-\langle y\rangle, e_{\mathcal{P}}} \leq \Re\right),
\end{aligned}
$$

$\mathbb{T}_{P, \mathrm{db}}(\mathbf{x}, \mathbf{y} ; \Re)=\hat{\mathcal{M}}_{P}^{\Lambda, 1,+}\left(\underline{\mathbf{x}}, \underline{\mathbf{y}} ; \mathrm{wt}_{-\langle y\rangle, e_{\mathcal{P}}} \leq \Re\right) \cap$

$$
\coprod_{z_{\lambda} \in \mathcal{P}^{\Lambda, \operatorname{deg}}} \coprod_{k=0}^{\infty} \operatorname{sign}\left(z_{\lambda}\right)(-1)^{k} \hat{\mathcal{M}}_{P, \lambda}^{0}\left(x_{\lambda}, z_{\lambda}\right) \times \hat{\mathcal{M}}_{P, \lambda}^{0}\left(z_{\lambda}, z_{\lambda}\right)^{\times k} \times \hat{\mathcal{M}}_{P, \lambda}^{0}\left(z_{\lambda}, y_{\lambda}\right)
$$

$$
\begin{gathered}
\mathbb{T}_{P, \mathrm{hs}-\mathrm{s}}(\mathbf{x}, \mathbf{y} ; \Re)=\hat{\mathcal{M}}_{P}^{\Lambda, 1,+}\left(\underline{\mathbf{x}}, \underline{\mathbf{y}} ; \mathrm{wt}_{-\langle y\rangle, e_{\mathcal{P}}} \leq \Re\right) \cap \\
\coprod_{i=0,1} \coprod_{\mathbf{z} \in \aleph_{\Lambda}}(-1)^{i} \hat{\mathcal{M}}_{P}^{S, i}(\mathbf{x}, \mathbf{z}) \times_{S} \hat{\mathcal{M}}_{P}^{S, 1-i}(\mathbf{z}, \mathbf{y}), \\
\mathbb{J}_{O}(S, \Re)=\coprod_{\lambda \in \partial S} \operatorname{sign}(\lambda: S) \hat{\mathcal{M}}_{O, \lambda}^{0}\left(\mathrm{wt}_{-\langle y\rangle, e_{\mathcal{P}}} \leq \Re\right), \\
\mathbb{T}_{O, \mathrm{db}}(\Re)=\hat{\mathcal{M}}_{O}^{\Lambda, 1,+}\left(\mathrm{wt}_{-\langle y\rangle, e_{\mathcal{P}}} \leq \Re\right) \cap \\
\coprod_{y_{\lambda} \in \mathcal{P}^{\Lambda, \mathrm{deg}}} \coprod_{k \in \mathbb{Z}^{+}} \operatorname{sign}\left(y_{\lambda}\right)(-1)^{k+\operatorname{ind}_{+}\left(y_{\lambda}\right)} \hat{\mathcal{M}}_{P, \lambda}^{0}\left(y_{\lambda}, y_{\lambda}\right)^{\times k}, \\
\mathbb{T}_{O, \mathrm{hs}-\mathrm{s}}(\Re)=\coprod_{\mathbf{x} \in \aleph_{\lambda}}(-1)^{\operatorname{ind}(\mathbf{x})+1} \hat{\mathcal{M}}_{P}^{S, 0}\left(\mathbf{x}, \mathbf{x} ; \mathrm{wt}_{-\langle y\rangle, e_{\mathcal{P}}} \leq \Re\right) .
\end{gathered}
$$


Remarks. $\mathbb{T}_{P, \mathrm{db}}, \mathbb{T}_{O, \mathrm{db}}$ consist of broken trajectories/orbits connected at a degenerate critical point (a death-birth); $\mathbb{T}_{P, \text { hs-s }}, \mathbb{T}_{O \text {,hs-s }}$ consist of oncebroken trajectories/orbits connected at a non-degenerate critical point, which occur with handle-slides.

The local structure of the parameterized moduli spaces of broken trajectories/orbits near $\mathbb{T}_{P, \mathrm{hs}-\mathrm{m}}, \mathbb{T}_{O, \text { hs-m }}$ is not understood. In fact, it seems that this is very sensitive to perturbations, and thus is not described by a universal formula. (See II.1.2.5 for more discussion of this difficulty from the perspective of gluing theory).

We shall show later (Section 6 and Part II) that under the assumptions of Theorem 4.1.1, there is an RHFS connecting the Floer systems associated with the pairs $\left(J_{1}, X_{1}\right),\left(J_{2}, X_{2}\right)$.

4.4. Invariance from RHFS. Let $\left\{\left(\mathcal{C}, \mathfrak{H}\right.\right.$, ind; $\left.\left.\mathfrak{y}_{\lambda}, \mathcal{V}_{\lambda}\right) \mid \lambda \in[1,2]\right\}$ be an RHFS. If $\left(\mathcal{C}, \mathfrak{H}\right.$, ind; $\left.y_{\lambda}, \mathcal{V}_{\lambda}\right)$ is a Floer system, let $\left(\mathrm{CF}\left(\mathcal{V}_{\lambda}\right), \partial_{\mathrm{F}}\left(\mathcal{V}_{\lambda}\right)\right)$ denote the associated Floer complex. Let $\eta_{\mathrm{F}}\left(\mathcal{V}_{\lambda}\right), \zeta_{\mathrm{F}}\left(\mathcal{V}_{\lambda}\right), I_{\mathrm{F}}\left(\mathcal{V}_{\lambda}\right)$, etc., be similarly defined. We are interested in how they vary with $\lambda$. By understanding this, we will see in this subsection that the existence of an RHFS satisfying some extra condition ((NEP) in 4.4.5 below), imply that $I_{\mathrm{F}}\left(\mathcal{V}_{1}\right)=I_{\mathrm{F}}\left(\mathcal{V}_{2}\right)$.

The condition (NEP) roughly says that the induced CHFSs on finitecyclic covers of $\mathcal{C}$ may be perturbed into RHFSs. It is needed because (RHFS*) says nothing about the structure of the parameterized moduli spaces near $\mathbb{T}_{P, \text { hs-m }}$ and $\mathbb{T}_{O \text {,hs-m }}$; thus from an RHFS one may only conclude the invariance of $I_{\mathrm{F}}$ "up to first order". By considering induced CHFSs on finite-cyclic covers of $\mathcal{C}$, the higher order contributions are recovered from the relation between torsion invariants of a space and its finite-cyclic covers.

The contents of this subsection are included only for the sake of completeness: they are straightforward consequences of (RHFS*), and are by no means new. We shall, therefore, leave out some details of the arguments which the reader may easily fill in, or find in the literature. A similar account with complete details may be found in [21].

4.4.1. Preparations. Recall that throughout an RHFS with c-class $\langle y\rangle$, the associated Novikov ring is $\Lambda_{\mathrm{F}}=\operatorname{Nov}(\operatorname{ker} \psi,-\langle y\rangle ; \mathbb{Z})$. Its ring of fractions $Q\left(\Lambda_{\mathrm{F}}\right)$ embeds via (3) into a Novikov ring, which we denote by $\Omega_{\mathrm{F}}$. Both $Q\left(\Lambda_{\mathrm{F}}\right)$ and $\Omega_{\mathrm{F}}$ decompose as finite direct sums of fields,

$$
Q\left(\Lambda_{\mathrm{F}}\right)=\bigoplus_{\kappa} F_{\kappa}, \quad \Omega_{\mathrm{F}}=\bigoplus_{\kappa} \mathbb{K}_{\kappa},
$$

where each $\kappa$ corresponds to an equivalence of characters $\kappa:$ Tors $(\operatorname{ker} \psi) \rightarrow$ $\mathbb{C}^{\times}$, each $\mathbb{K}_{\kappa}$ is again a Novikov ring, and the embedding (3) is compatible with the decomposition.

For the invariance proof, it is convenient to work with the Novikov ring $\Omega_{\mathrm{F}}$ instead of $Q(\Lambda)$, namely, we identify $\mathfrak{Q}$ as an element in $\Omega_{\mathrm{F}}$ via the 
embedding (3), for $\mathfrak{Q}=\partial_{\mathrm{F}}, \tau_{\mathrm{F}}, \eta_{\mathrm{F}}, \zeta_{\mathrm{F}}$, or $I_{\mathrm{F}}$. It is also sometimes convenient to work with a field component of $\Omega_{\mathrm{F}}$ at a time. In such case, we denote by $\mathfrak{Q}^{\kappa}=\mathfrak{Q} \otimes_{\Lambda_{\mathrm{F}}} \mathbb{K}_{\kappa}$, namely, the image of $\mathfrak{Q}$ under the composition of (3) and the projection to the field component $\mathbb{K}_{\kappa}$. Of course, invariance of $I_{\mathrm{F}}^{\kappa}$ for each $\kappa$ will imply invariance of the total $I_{\mathrm{F}}$.

Given $a=\sum_{g} a_{g} g \in \operatorname{Nov}(G, N ; R)$, let

$$
\mathrm{Tc}_{N ; \Re}[a]:=\sum_{g \in G, N(g) \leq \Re} a_{g} g .
$$

Choose an ordered basis $e_{\mathcal{P}}$ for each $\mathrm{CF}\left(\mathcal{V}_{\lambda}\right)$ as in Sections 2.2 .3 and 4.3, thus identifying $\partial_{\mathrm{F}}\left(\mathcal{V}_{\lambda}\right)=\left(\partial_{\mathrm{F}}\left(\mathcal{V}_{\lambda}\right)_{i j}\right)$ with a matrix with entries in $\Omega_{\mathrm{F}}$. We will use $\mathrm{Tc}_{-\langle y\rangle ; \Re}\left[\partial_{\mathrm{F}}\left(\mathcal{V}_{\lambda}\right)\right]$ to denote the matrix with entries $\mathrm{Tc}_{-\langle y\rangle ; \Re}\left[\partial_{\mathrm{F}}\left(\mathcal{V}_{\lambda}\right)_{i j}\right]$. With the basis so fixed, $\tau_{\mathrm{F}}\left(\mathcal{V}_{\lambda}\right)$ and $I_{\mathrm{F}}\left(\mathcal{V}_{\lambda}\right)$ will now be viewed as elements in the Novikov ring $\Omega_{\mathrm{F}}$.

In contrast, let $\partial_{\mathrm{F}}\left(\mathcal{V}_{\lambda} ; \Re\right), \eta_{\mathrm{F}}\left(\mathcal{V}_{\lambda} ; \Re\right)$ be defined in the same way as $\partial_{\mathrm{F}}, \eta_{\mathrm{F}}$ in Section 2.2 using weight-truncated versions of moduli:

$$
\begin{aligned}
\partial_{\mathrm{F}}\left(\mathcal{V}_{\lambda} ; \Re\right)_{x_{\lambda}, y_{\lambda}} & :=\sum_{A \in \mathfrak{H}} \chi\left(\hat{\mathcal{M}}_{P, \lambda}^{0}\left(\operatorname{Lf}\left(x_{\lambda}\right), A \cdot \operatorname{Lf}\left(y_{\lambda}\right) ; \mathrm{wt}_{-\langle y\rangle, e_{\mathcal{P}}} \leq \Re\right)\right)[A]_{\text {ker } \psi} ; \\
\eta_{\mathrm{F}}\left(\mathcal{V}_{\lambda} ; \Re\right) & \left.:=\sum_{A \in \operatorname{ker} \psi} \chi\left(\hat{\mathcal{M}}_{O, \lambda}^{0}(A) ; \mathrm{wt}_{-\langle y\rangle, e_{\mathcal{P}}} \leq \Re\right)\right) A .
\end{aligned}
$$

4.4.2. Invariance in regular range and left/right limits. Below are some simple consequences of the smoothness and compactness properties of the parameterized moduli spaces in (RHFS2, 3). Let

$$
\begin{aligned}
\Lambda_{\text {reg }} & :=\Lambda \backslash\left(\Lambda_{h s} \cup \Lambda_{\mathrm{db}}\right) ; \\
\Lambda_{\text {floer }} & :=\left\{\lambda \mid \lambda \in \Lambda, \nu_{\lambda} \text { generates a Floer system }\right\} .
\end{aligned}
$$

\section{Lemma.}

(a) $\Lambda_{\text {floer }} \subset \Lambda_{\text {reg }} \subset \Lambda$ is Baire.

(b) $\partial_{\mathrm{F}}\left(\mathcal{V}_{\lambda} ; \Re\right)$ is well-defined and locally constant for $\lambda$ in $\Lambda \backslash\left(\Lambda_{h s}^{\Re} \cup \Lambda_{\mathrm{db}}\right)$. $\eta_{\mathrm{F}}\left(\mathcal{V}_{\lambda} ; \Re\right)$ is well-defined and locally constant for $\lambda$ in $\Lambda \backslash\left(\Lambda_{h s: i i}^{\Re} \cup \Lambda_{\mathrm{db}}\right)$.

(c) For $\mathfrak{Q}=\partial_{\mathrm{F}}, \eta_{\mathrm{F}}, \tau_{\mathrm{F}}, \zeta_{\mathrm{F}}$, or $I_{\mathrm{F}}, \mathfrak{Q}\left(\mathcal{V}_{\lambda}\right)$ is well-defined for all $\lambda \in \Lambda_{\text {reg. }}$. In fact, for $\mathfrak{Q}=\partial_{\mathrm{F}}$ or $\eta_{\mathrm{F}}, \mathfrak{Q}\left(\mathcal{V}_{\lambda} ; \Re\right)=\mathrm{Tc}_{-}\langle y\rangle ; \Re\left[\mathfrak{Q}\left(\mathcal{V}_{\lambda}\right)\right]$ for any $\lambda \in \Lambda_{\text {reg. }}$.

(d) The statements (i)-(iii) below hold for $\mathfrak{Q}=\partial_{\mathrm{F}}, \eta_{\mathrm{F}}, \zeta_{\mathrm{F}}$. They also hold for $\mathfrak{Q}^{\kappa}=\tau_{\mathrm{F}}^{\kappa}, I_{\mathrm{F}}^{\kappa}$ under the additional condition that

$$
H_{*}\left(\mathrm{CF}\left(\mathcal{V}_{\lambda}\right)^{\kappa}\right)=0 \quad \forall \lambda \in \Lambda_{\text {reg }},
$$

(i) For all $\lambda \in \Lambda$, the following left and right limits are well-defined.

$$
\mathfrak{Q}_{\lambda-}:=\lim _{\lambda^{\prime} \in \Lambda_{\mathrm{reg}}, \lambda^{\prime} \nearrow \lambda} \mathfrak{Q}\left(\mathcal{V}_{\lambda^{\prime}}\right), \quad \mathfrak{Q}_{\lambda+}:=\lim _{\lambda^{\prime} \in \Lambda_{\mathrm{reg}}, \lambda^{\prime} \searrow \lambda} \mathfrak{Q}\left(\mathcal{V}_{\lambda^{\prime}}\right) \text {. }
$$

(ii) $\mathfrak{Q}_{\lambda-}=\mathfrak{Q}_{\lambda+}=\mathfrak{Q}\left(\mathcal{V}_{\lambda}\right)$ when $\lambda \in \Lambda_{\text {reg }}$. 
(iii) For all sufficiently large $\Re, \mathrm{Tc}_{-\langle y\rangle ; \Re}\left[\mathfrak{Q}_{\lambda-}\right]=\mathrm{Tc}_{-\langle y\rangle ; \Re}\left[\mathfrak{Q}_{\lambda+}\right]$ is locally constant on $\Lambda \backslash\left(\Lambda_{\mathrm{db}} \cup \Lambda_{h s}^{2 \Re}\right)$.

Sketch of proof. Given $\mathbf{x}, \mathbf{y} \in \aleph_{\Lambda}$, and $[a, b] \in \Lambda \backslash\left(\Lambda_{\mathrm{db}} \cup \Lambda_{h s}^{\Re}\right)$, (RHFS2) implies that $\hat{\mathcal{M}}_{P}^{[a, b], 1}\left(\mathbf{x}, \mathbf{y} ; \mathrm{wt}_{-\langle y\rangle, \text { ep }} \leq \Re\right)$ is a compact 1-manifold with boundary $\hat{\mathcal{M}}_{P, b}^{0}\left(x_{b}, y_{b} ; \mathrm{wt}_{-\langle y\rangle, e_{\mathcal{P}}} \leq \Re\right)-\hat{\mathcal{M}}_{P, a}^{0}\left(x_{a}, y_{a} ; \mathrm{wt}_{-\langle y\rangle, e_{\mathcal{P}}} \leq \Re\right)$. Namely, the parameterized moduli space forms a cobordism between $\hat{\mathcal{M}}_{P, b}^{0}$ and $\hat{\mathcal{M}}_{P, a}^{0}$.

Given $\lambda \in[a, b]$, the fiber over $\lambda$ of $\Pi_{\Lambda}: \hat{\mathcal{M}}_{P}^{[a, b], 1}\left(\mathbf{x}, \mathbf{y} ; \mathrm{wt}_{-\langle y\rangle, e_{\mathcal{P}}} \leq \Re\right) \rightarrow \Lambda$ is $\hat{\mathcal{M}}_{P, \lambda}^{0}\left(x_{\lambda}, y_{\lambda} ; \mathrm{wt}_{-\langle y\rangle, e_{\mathcal{P}}} \leq \Re\right)$, which is Zariski smooth and compact unless $\lambda$ is a critical value of $\Pi_{\Lambda}$. (This is because ker $E_{u_{\lambda}} \subset \operatorname{ker} D \Pi_{\lambda}$, where $E_{u_{\lambda}}$ is the deformation operator of the fiber at $u_{\lambda}$.) By Sard's theorem, noncritical values form a Baire set. Claim (a) follows from this observation and its analog for $\hat{\mathcal{M}}_{O}$.

On the other hand, notice that one does not need $\lambda \in \Lambda_{\text {floer }}$ for $\partial_{\mathrm{F}}\left(\mathcal{V}_{\lambda} ; \Re\right), \eta_{\mathrm{F}}\left(\mathcal{V}_{\lambda} ; \Re\right)$ to be well-defined. Their definitions involve only Euler characteristics of various moduli spaces $\hat{\mathcal{M}}_{\lambda}^{0}$, and as long as there is a smooth compact parameterized moduli space $\Pi_{\Lambda}: \hat{\mathcal{M}}^{[a, b], 1} \rightarrow[a, b]$ containing $\hat{\mathcal{M}}_{\lambda}^{0}$ as a fiber, $\chi\left(\hat{\mathcal{M}}_{\lambda}^{0}\right)$ is well-defined and constant in $\lambda \in[a, b]$. Claims (b) and (c) then follow.

To justify (d), one just needs to show that the terms in $\mathrm{Tc}_{-\langle y\rangle ; \Re}\left[\mathfrak{Q}_{\lambda}\right]$ $\left(\lambda \in \Lambda_{\text {reg }}\right)$ only depend on Euler characteristics of moduli spaces with weighttruncation $\mathrm{wt}_{-\langle y\rangle, e_{\mathcal{P}}} \leq 2 \Re$. The local constancy of $\mathrm{Tc}_{-\langle y\rangle ; \Re}\left[\mathfrak{Q}_{\lambda}\right]$ in $\lambda$, and hence the existence of left/right limits, would then follow from the same cobordism argument used for (b).

For $\mathfrak{Q}=\partial_{\mathrm{F}}, \eta_{\mathrm{F}}, \zeta_{\mathrm{F}}$, this is clear from the definitions; in fact, only flows with $\mathrm{wt}_{-\langle y\rangle, e_{\mathcal{P}}} \leq \Re$ contribute.

The story with $\mathfrak{Q}=\tau_{\mathrm{F}}^{\kappa}, I_{\mathrm{F}}^{\kappa}$ is less straightforward and requires the assumption that $\Re$ is sufficiently large. We shall concentrate on $\mathfrak{Q}=\tau_{\mathrm{F}}^{\kappa}$, since the case of $\mathfrak{Q}=I_{\mathrm{F}}^{\kappa}$ follows from the cases of $\mathfrak{Q}=\tau_{\mathrm{F}}^{\kappa}$ and $\mathfrak{Q}=\zeta_{\mathrm{F}}$.

First, note that by the compactness of $\hat{\mathcal{M}}_{P}^{\Lambda, 1}\left(\mathrm{wt}_{-\langle y\rangle, e_{\mathcal{P}}} \leq \Re\right)$, there is a constant $C>0$ such that

$$
\mathrm{wt}_{-\langle y\rangle, e_{\mathcal{P}}}\left(u_{\lambda}\right) \geq-C \quad \forall u_{\lambda} \in \hat{\mathcal{M}}_{P}^{\Lambda, 1} .
$$

Write $\Lambda \backslash \Lambda_{\mathrm{db}}=\coprod_{k} S_{k}$, where each $S_{k}$ is a path component of $\Lambda \backslash \Lambda_{\mathrm{db}}$. The Floer groups $\mathrm{CF}\left(\mathcal{V}_{\lambda}\right)$ are independent of $\lambda$ on each $S_{k}$. By the acyclicity of $\left(\mathrm{CF}\left(\mathcal{V}_{\lambda}\right)^{\kappa}, \partial_{\mathrm{F}}\left(\mathcal{V}_{\lambda}\right)^{\kappa}\right)$, there is a splitting $\mathrm{CF}_{i}^{\kappa}=A_{i}^{\kappa} \oplus B_{i}^{\kappa}, A_{i}^{\kappa}, B_{i}^{\kappa}$ being each spanned by elements in $\mathcal{P}$, such that the submatrices $\underline{\partial}_{i}^{\kappa}:=\partial_{\mathrm{F}}^{\kappa}: A_{i}^{\kappa} \rightarrow B_{i-1}^{\kappa}$ are isomorphisms. (See [23] Lemma 2.7.) By the local constancy of $\partial_{\mathrm{F}}\left(\mathcal{V}_{\lambda} ; \Re\right)$ and $\left(\mathrm{RHFS}^{*}\right)$ (see also Lemmas 4.4.4, 4.4.5 below), $A_{i}^{\kappa}$ and $B_{i}^{\kappa}$ may be chosen independently of $\lambda$ in each $S_{k}$. Furthermore, $\operatorname{deg}\left(\underline{\partial}_{i}^{\kappa}\right)$ is well 
defined and constant for each $\lambda$ in $S_{k}$; we denote this value by $\mathbb{M}_{i, k}$. Let $\mathbb{M}:=\sup _{k} \sum_{i}\left|\mathbb{M}_{i, k}\right|$.

By (RHFS2), we may require $\Re$ to be large enough so that the square matrices $\partial_{\mathrm{F}}\left(\mathcal{V}_{\lambda} ; \Re\right)^{\kappa}: A_{i}^{\kappa} \rightarrow B_{i-1}^{\kappa}$ are isomorphisms for all $i$ and $\lambda \in$ $\Lambda \backslash\left(\Lambda_{\mathrm{db}} \cup \Lambda_{h s}^{\Re}\right)$. In addition, suppose

$$
\Re>2 \mathbb{M}+C \cdot \#\left(\aleph_{\Lambda}\right) .
$$

According to $[\mathbf{2 3}]$ Lemma $2.7, \tau_{\mathrm{F}}^{\kappa}=\prod_{i}\left(\operatorname{det} \underline{\partial}_{i}^{\kappa}\right)^{(-1)^{i}}$. Expanding, we see that for $\lambda \in S_{k}$, the degree of any term in $\tau_{\mathrm{F}}\left(\mathcal{V}_{\lambda}\right)^{\kappa}$ can be written as $\sum_{i}(-1)^{i} \mathbb{M}_{i, k}$ plus a sum of non-negative terms of the form

$$
\sum_{j=1}^{\operatorname{dim} A_{i}} \mathrm{wt}_{-y_{, e_{\mathcal{P}}}}\left(u_{j}\right)-\mathbb{M}_{i, k},
$$

where $u_{j}$ are flows from an index i critical point to an index $i-1$ critical point. The constraint that $\operatorname{deg} \leq \Re$ for any term in $\operatorname{Tc}_{-}\langle y\rangle ; \Re\left[\tau_{\mathrm{F}}\left(\mathcal{V}_{\lambda}\right)^{\kappa}\right]$, together with (36) and (35) then imply that wt_y,e⿻上丨 $\left(u_{j}\right) \leq 2 \Re$ for any flow $u_{j}$ contributing to $\mathrm{Tc}_{-\langle y\rangle ; \Re}\left[\tau_{\mathrm{F}}\left(\mathcal{V}_{\lambda}\right)^{\kappa}\right]$.

4.4.3. At a death-birth bifurcation. We shall focus on the case of a death, since the case of a birth may be obtained from the case of a death by reversing the orientation of $\Lambda$.

Suppose $z_{\lambda} \in \mathcal{P}^{\Lambda, \operatorname{deg}}$ is a death with $\operatorname{ind}_{+}\left(z_{\lambda}\right)=i$. Let $\lambda_{-}, \lambda_{+} \in \Lambda_{\text {floer }}$ be close to $\lambda$ with $\lambda_{-}<\lambda<\lambda_{+}$. By $\left(\mathrm{RHFS1}^{*}\right), \mathcal{P}_{\lambda_{-}}=\mathcal{P}_{\lambda_{+}} \sqcup\left\{z_{\lambda_{-}}^{+}\right\} \sqcup\left\{z_{\lambda_{-}}^{-}\right\}$, where $\operatorname{ind}\left(z_{\lambda_{-}}^{+}\right)=i, \operatorname{ind}\left(z_{\lambda_{-}}^{-}\right)=i-1$. Thus,

$\mathrm{CF}_{i}\left(\mathcal{V}_{\lambda_{-}}\right)=\mathbb{R}\left\{z_{\lambda_{-}}^{+}\right\} \oplus \mathrm{CF}_{i}\left(\mathcal{V}_{\lambda_{+}}\right) ; \quad \mathrm{CF}_{i-1}\left(\mathcal{V}_{\lambda_{-}}\right)=\mathbb{R}\left\{z_{\lambda_{-}}^{-}\right\} \oplus \mathrm{CF}_{i-1}\left(\mathcal{V}_{\lambda_{+}}\right)$.

Since the left and right limits $\mathrm{CF}_{\lambda_{ \pm}, i}=\mathrm{CF}_{i}\left(\mathcal{V}_{\lambda_{ \pm}}\right)$, they have similar decompositions.

The properties (RHFS2*, $\left.3^{*}, 4\right)$, especially those describing the structures of the moduli spaces near $\mathbb{T}_{P, \mathrm{db}}$ and $\mathbb{T}_{O, \mathrm{db}}$, lead to:

\section{Lemma.}

(a) In terms of the above decomposition of $\mathrm{CF}_{\lambda-, i}, \mathrm{CF}_{\lambda-, i-1}$, the left limit $\left(\partial_{\mathrm{F}, \lambda-}\right)_{i}: \mathrm{CF}_{\lambda-, i} \rightarrow \mathrm{CF}_{\lambda-, i-1}$ is:

$$
\left(\partial_{\mathrm{F}, \lambda-}\right)_{i}=\left(\begin{array}{cc}
1+b & v \\
w & N
\end{array}\right)
$$

where

$$
\begin{aligned}
b & =\sum_{A \in \operatorname{ker} \psi} \chi\left(\hat{\mathcal{M}}_{P, \lambda}^{0}\left(\operatorname{Lf}\left(z_{\lambda}\right), \operatorname{Lf}\left(z_{\lambda}\right)\right) A=\sum_{j} b_{j}\right. \\
b_{j} & =\operatorname{sign}\left(u_{j}\right)\left[u_{j}\right]_{\text {ker } \psi} \quad \text { where } u_{j} \in \hat{\mathcal{M}}_{P}^{0}\left(z_{\lambda}, z_{\lambda}\right), u_{j} \neq z_{\lambda},
\end{aligned}
$$


$v=\left(v_{y_{\lambda}}\right), w=\left(w_{x_{\lambda}}\right), N=\left(N_{x_{\lambda}, y_{\lambda}}\right)$ are, respectively, a row vector, a column vector, and a matrix, with entries given by

$$
\begin{aligned}
v_{y_{\lambda}} & =\sum_{A \in \mathfrak{H}} \chi\left(\hat{\mathcal{M}}_{P, \lambda}^{0}\left(\operatorname{Lf}\left(z_{\lambda}\right), A \cdot \operatorname{Lf}\left(y_{\lambda}\right)\right)[A]_{\text {ker } \psi,},\right. \\
w_{x_{\lambda}} & =\sum_{A \in \mathfrak{H}} \chi\left(\hat{\mathcal{M}}_{P, \lambda}^{0}\left(\operatorname{Lf}\left(x_{\lambda}\right), A \cdot \operatorname{Lf}\left(z_{\lambda}\right)\right)[A]_{\operatorname{ker} \psi},\right. \\
N_{x_{\lambda}, y_{\lambda}} & =\sum_{A \in \mathfrak{H}} \chi\left(\hat{\mathcal{M}}_{P, \lambda}^{0}\left(\operatorname{Lf}\left(x_{\lambda}\right), A \cdot \operatorname{Lf}\left(y_{\lambda}\right)\right)[A]_{\operatorname{ker} \psi},\right.
\end{aligned}
$$

$x_{\lambda}, y_{\lambda} \in \mathcal{P}_{\lambda}$ being non-degenerate critical points with indices $i$ and $i-1$, respectively. On the other hand,

$$
\left(\partial_{\mathrm{F}, \lambda+}\right)_{i}=N+\sum_{k=0}^{\infty}(-1)^{k+1} w b^{k} v,
$$

(b) $\left(\partial_{\mathrm{F}, \lambda-}\right)_{k}=\left(\partial_{\mathrm{F}, \lambda+}\right)_{k}$ when $k \neq i, i \pm 1$. When $k=i+1$ or $i-1$, $\left(\partial_{\mathrm{F}, \lambda+}\right)_{k}$ is obtained from $\left(\partial_{\mathrm{F}, \lambda-}\right)_{k}$ by deleting the row corresponding to $z_{\lambda-}$, or the column corresponding to $z_{\lambda+}$, respectively.

(c) We have

$$
\eta_{\mathrm{F}, \lambda+}-\eta_{\mathrm{F}, \lambda-}=\sum_{k=1}^{\infty} \sum_{i_{1}, \ldots, i_{k}=1}^{\infty} \frac{(-1)^{k+i+1}}{k} b_{i_{1}} \cdots b_{i_{k}} .
$$

(d) Hence, by elementary algebra

$$
\begin{aligned}
\tau_{\mathrm{F}, \lambda+} / \tau_{\mathrm{F}, \lambda-} & =(1+b)^{(-1)^{i+1}} \\
\zeta_{\mathrm{F}, \lambda+} / \zeta_{\mathrm{F}, \lambda-} & =(1+b)^{(-1)^{i}} \\
I_{\mathrm{F}, \lambda+} & =I_{\mathrm{F}, \lambda-} .
\end{aligned}
$$

\section{Remarks.}

(a) By (RHFS2), the entries of $b, v, w, N$ all take values in $\Lambda_{\mathrm{F}}$.

(b) Let $\mathbf{e}_{i j}$ denote a square matrix with the $i j$-th entry 1 , and 0 for all other entries. We refer to the conjugation action by an elementary matrix of the form $\mathrm{Id}+\mathbf{e}_{i j}$ as an elementary transformation. By an expansion, we mean the direct sum with the rank 1 identity matrix, and a collapse refers to the converse operation. When $\langle y\rangle=0, b=0$, and the above lemma shows that in this case the left and right limits $\partial_{\mathrm{F}, \lambda-}, \partial_{\mathrm{F}, \lambda+}$ are related by expansion/collapse modulo elementary transformations.

4.4.4. At a Type I handle-slide. Let $u_{\lambda} \in \hat{\mathcal{M}}_{P}^{\Lambda, 0}(\mathbf{x}, \mathbf{y})$, where $\mathbf{x} \neq \mathbf{y}$. Let $T=\oplus_{i} T_{i}$, where $T_{i}: \mathrm{CF}_{i}\left(\mathcal{V}_{\lambda}\right) \rightarrow \mathrm{CF}_{i}\left(\mathcal{V}_{\lambda}\right)$ is

$$
T_{i}= \begin{cases}I d & \text { when } i \neq \operatorname{ind} x_{\lambda}, \\ I d+\operatorname{sign}\left(u_{\lambda}\right)\left[u_{\lambda}\right]_{\operatorname{ker} \psi} \mathbf{e}_{x_{\lambda}, y_{\lambda}} & \text { when } i=\operatorname{ind} x_{\lambda}=\operatorname{ind} y_{\lambda} .\end{cases}
$$


Lemma. In this case,

(a) $\partial_{\mathrm{F}, \lambda+}=T \partial_{\mathrm{F}, \lambda-} T^{-1}$.

(b) $\eta_{\mathrm{F}, \lambda+}=\eta_{\mathrm{F}, \lambda-}$.

(c) Hence, $\mathfrak{Q}_{\lambda+}=\mathfrak{Q}_{\lambda-}$ for $\mathfrak{Q}=\zeta_{\mathrm{F}}, \tau_{\mathrm{F}}, I_{\mathrm{F}}$.

This is again a consequence of $\left(\mathrm{RHFS}^{*}, 3^{*}, 4\right)$ : (a) follows from the description of the structure of the moduli spaces near $\mathbb{T}_{P, \mathrm{hs} \text { s }}$, and (b) follows from the fact that in this case, $\lambda \in \Lambda_{h s} \backslash \Lambda_{h s: i i}$, and hence $\Pi_{\Lambda}^{-1}(\lambda)$ $\cap \partial \hat{\mathcal{M}}_{O}^{\Lambda, 1,+}=\emptyset$.

4.4.5. At a Type II handle-slide. Up to "first order", this can be described similar to Section 4.4 .4 above. To understand the higher order contributions, we need to further assume (NEP) below to apply Hutchings's argument. Some preparation is required to state the assumption (NEP).

For convenience, we introduce the following weaker version of RHFS: a homotopy of formal flows is $R$-regular with respect to a weight truncation $\mathrm{wt}_{-\langle y\rangle, e_{\mathfrak{p}}}$, if in addition to (RHFS1*), the statements in $\left(\mathrm{RHFS}^{*}, 3^{*}, 4\right)$ are only required to hold for all weight-truncated versions

$$
\hat{\mathcal{M}}_{P}^{0}\left(x_{\lambda}, y_{\lambda} ; \mathrm{wt}_{-\langle y\rangle, e_{\mathcal{P}}} \leq \Re\right), \hat{\mathcal{M}}_{P}^{S, k}\left(\mathbf{x}, \mathbf{y} ; \mathrm{wt}_{-\langle y\rangle, e_{\mathcal{P}}} \leq \Re\right), \hat{\mathcal{M}}_{O}^{S, k}\left(\mathrm{wt}_{-y_{, e_{\mathcal{P}}}} \leq \Re\right)
$$

with $\Re \leq R$. Notice that we do not require an $R$-regular homotopy to be a CHFS.

A finite-cyclic cover of $\mathcal{C}$ is said to be $\mathfrak{H}$-adapted if it is a subcover of $\tilde{\mathcal{C}}$, and its monodromy factors as

$$
\pi_{m} \circ \nu \circ \mathrm{im}: \pi_{1}(\mathcal{C}) \rightarrow \mathbb{Z} / m \mathbb{Z},
$$

where $\nu$ is a homomorphism $\nu: \mathfrak{H} \rightarrow \mathbb{Z}$, and $\pi_{m}$ is the epimorphism $\mathbb{Z} \rightarrow \mathbb{Z} / m \mathbb{Z}$. We shall denote the $m$-cyclic cover of such monodromy by $\mathrm{e}^{\nu, m}$.

Let $u_{\lambda} \in \hat{\mathcal{M}}_{P}^{\Lambda, 0}(\operatorname{Lf}(\mathbf{x}), A \cdot \operatorname{Lf}(\mathbf{x}))$ be a Type II handle-slide. Let $\operatorname{div}(A)$ denote the divisibility of $A \in \operatorname{ker} \psi \subset \mathfrak{H}$.

An $\mathfrak{H}$-adapted $m$-cyclic cover, $\mathrm{e}^{\nu, m}$, is said to be $u_{\lambda}$-breaking if $\nu(A)=$ $\operatorname{div}(A)$, and $m>1$ does not $\operatorname{devide} \operatorname{div}(A)$. Indeed, in this case the end points of $u_{\lambda}$ lift to different points in $\mathrm{C}^{\nu, m}$.

We are now ready to state the assumption. Let $\left\{\left(\mathcal{C}, \mathfrak{H}, \text { ind }, y_{\lambda}, \mathcal{V}_{\lambda}\right)\right\}_{\lambda \in \Lambda}$ be an RHFS, and $\mathrm{wt}_{-\langle y\rangle, e_{\mathcal{P}}}$ be a weight filtration adapted to this RHFS. Let $u_{\lambda} \in \hat{\mathcal{M}}_{P}^{\Lambda, 0}(\operatorname{Lf}(\mathbf{x}), A \cdot \operatorname{Lf}(\mathbf{x}))$ be a Type II handle-slide.

(NEP) EXISTENCE OF NON-EQUIVARIANT PERTURBATIONS. We say that (NEP) holds for $u_{\lambda}$ if, given:

- an arbitrarily large $\Re \in \mathbb{R}^{+}$,

- a small neighborhoods $S_{\lambda}^{\prime}, S_{\lambda} \subset \Lambda$ about $\lambda$, such that $S_{\lambda}^{\prime}$ is a proper sub-interval of $S_{\lambda}$, and $\hat{\mathcal{M}}_{P}^{S_{\lambda}, 0}\left(\mathrm{wt}_{-\langle y\rangle, e_{\mathcal{P}}} \leq \Re\right)$ contains an unique element, $u_{\lambda}$, 
- an arbitrary $u_{\lambda}$-breaking, $\mathfrak{H}$-adapted finite-cyclic cover $\mathrm{e}^{\nu, m}$, there is an $\Re$-regular homotopy of formal flows, $\left\{\mathcal{V}_{\lambda}^{+} \mid \lambda \in S_{\lambda}\right\}$ over $\mathrm{C}^{\nu, m}$, such that $\mathcal{V}_{\lambda}^{+}$agrees with the lift of $\mathcal{V}_{\lambda}$ to $\mathrm{e}^{\nu, m} \forall \lambda \in \Lambda \backslash S_{\lambda}^{\prime}$.

Lemma. Assuming that (NEP) holds for $u_{\lambda} \in \hat{\mathcal{M}}_{P}^{\Lambda, 0}(\operatorname{Lf}(\mathbf{x}), A \cdot \operatorname{Lf}(\mathbf{x}))$. Then

(a) $\partial_{\mathrm{F}, \lambda+}=T \partial_{\mathrm{F}, \lambda-} T^{-1}$, where $T=\oplus_{i} T_{i}, T_{i}: \mathrm{CF}_{i}\left(\mathcal{V}_{\lambda}\right) \rightarrow \mathrm{CF}_{i}\left(\mathcal{V}_{\lambda}\right)$ being

$$
T_{i}= \begin{cases}I d & \text { when } i \neq \operatorname{ind} x_{\lambda}, \\ I d+\chi \mathbf{e}_{x_{\lambda}, x_{\lambda}} & \text { when } i=\operatorname{ind} x_{\lambda},\end{cases}
$$

and $\chi=\operatorname{sign}(u)[u]+\sum_{j=2}^{\infty} \chi_{j}[u]^{j} \in \operatorname{Nov}^{+}(\operatorname{ker} \psi,-\langle y\rangle ; \mathbb{Z})$.

(b) $\eta_{\mathrm{F}, \lambda+}-\eta_{\mathrm{F}, \lambda-}=\chi^{\prime}$, where $\chi^{\prime}=(-1)^{\text {ind } x} \operatorname{sign}(u)[u]+\sum_{j=2}^{\infty} \chi_{j}^{\prime}[u]^{j} \in$ $\mathrm{Nov}^{+}(\operatorname{ker} \psi,-\langle y\rangle ; \mathbb{Z})$.

(c) $\tau_{\mathrm{F}, \lambda+}=(1+\chi)^{(-1)^{\text {ind } x+1}} \tau_{\mathrm{F}, \lambda-}$.

(d) $I_{\mathrm{F}, \lambda+}=I_{\mathrm{F}, \lambda-}$.

Sketch of proof.

(a) follows from the description of the structure of the parameterized moduli space of broken trajectories near $\mathbb{T}_{P \text {,hs-s }}$ in (RHFS2c, 4), the property (NEP), and the proof of [21] Lemma 3.7.

(b) is a consequence of the description of the structure of parameterized moduli space of broken orbits near $\mathbb{T}_{O, \text { hs-s }}$ in (RHFS3c, 4).

(c) follows from (a) by elementary algebra, and (b), (c) together imply $I_{\mathrm{F}, \lambda-}=c I_{\mathrm{F}, \lambda+}$, where $c=1+\sum_{i=2}^{\infty} c_{i}[u]^{i}$.

Now by (NEP), the proof of $[\mathbf{2 1}]$ Lemma 3.10 can be applied to verify $(d)$.

4.4.6. Conclusion. Summarizing the above, we state:

Proposition. Let $\left\{\mathcal{V}_{\lambda}\right\}_{\lambda \in[1,2]}$ be an RHFS such that (NEP) holds for all Type II handle-slide bifurcations. Then $I_{\mathrm{F}}\left(\mathcal{V}_{1}\right)=I_{\mathrm{F}}\left(\mathcal{V}_{2}\right)$.

More precisely, $I_{\mathrm{F}}\left(\mathcal{V}_{i}\right)$ needs to be replaced by $i_{-\langle y\rangle} I_{\mathrm{F}}\left(\mathcal{V}_{i}\right)$ when $\left\langle y_{i}\right\rangle=0$ for the above equality to make sense.

Proof. We shall show that $I_{\mathrm{F}}\left(\mathcal{V}_{1}\right)^{\kappa}=I_{\mathrm{F}}\left(\mathcal{V}_{2}\right)^{\kappa}$ for each $\kappa$.

It follows from the invariance of Floer homology that either $H_{*}\left(\mathrm{CF}\left(\mathcal{V}_{\lambda}\right)^{\kappa}\right)$ $=0 \forall \lambda \in \Lambda_{\text {reg }}$, or $H_{*}\left(\mathrm{CF}\left(\mathcal{V}_{\lambda}\right)^{\kappa}\right) \neq 0 \forall \lambda \in \Lambda_{\text {reg. }}$. (cf. [32]. Alternatively, one may derive it from the description of bifurcation behaviors in Section 4.4.34.4.5). In the latter case, $I_{\mathrm{F}}\left(\mathcal{V}_{1}\right)^{\kappa}=I_{\mathrm{F}}\left(\mathcal{V}_{2}\right)^{\kappa}=0$ and we are done. Thus, we may assume $H_{*}\left(\mathrm{CF}\left(\mathcal{V}_{\lambda}\right)^{\kappa}\right)=0 \forall \lambda \in \Lambda_{\text {reg. }}$. The invariance of $I_{\mathrm{F}}^{\kappa}$ then follows from Lemma 4.4.2 (d) and the comparison of left and right limits at bifurcation points in Lemmas 4.4.3-4.4.5. 
With this Proposition in place, the proof of Theorem 4.1.1 then consists in showing that the conditions of Theorem 4.1.1 imply the existence of an RHFS with property (NEP), that connects the Floer systems associated to $\left(J_{1}, X_{1}\right),\left(J_{2}, X_{2}\right)$. This is the goal of the rest of this paper. First, to obtain (RHFS2d) we need to generalize the structural results for $\mathcal{M}_{P}(x, y)$ in Section 3.2 to case where one or both of the critical points $x, y$ are degenerate. This is done in the next section. In Section 6 , we show that the conditions of Theorem 4.1.1 imply the existence of certain CHFSs satisfying all the properties (RHFS*) except for (RHFS2c, 3c, 4), called "admissible $(J, X)$-homotopies". It will be shown in part II that the remaining properties (RHFS2c, 3c, 4), (NEP) can be derived from the definition of admissible $(J, X)$-homotopies. This will then conclude the proof of Theorem 4.1.1.

\section{Near a minimally degenerate critical point $y$}

We now return to the context of the specific version of Floer theory described in Section 3. This section contains detailed description of the behaviors of various flows in the loop space near a minimally degenerate critical point $y$ : In Section 5.1 we give decay estimates of a flow in $\mathcal{M}_{P}$ ending at $y$; in Section 5.2, these estimates are applied to obtain the structure theory of moduli spaces such as $\mathcal{M}_{P}(x, y)$; in Section 5.3 we assume that $y$ belongs to a family $y \in \mathcal{P}^{\Lambda}$ for a homotopy of Floer systems generated by a 1 parameter family $\left\{\left(J_{\lambda}, X_{\lambda}\right)\right\}_{\lambda \in \Lambda}$, and describe the neighborhood of $y$ in $\mathcal{P} \Lambda$. These results will be useful for verifying the property (RHFS2d), and the estimates will be important for the proofs of the gluing theorems in Part II.

5.1. A decay estimate for the flows. Following [36], we use center manifold theory on Hilbert manifolds to describe the asymptotics of flows ending at $y$ (Proposition 5.1.3 below).

5.1.1. Preparations. Some preliminaries are required to state the assumptions of the Proposition.

First, notice that for any (possibly degenerate) $(y,[v]) \in \tilde{\mathcal{P}}$, the generalized Conley-Zehnder index ind $\tilde{n}^{\sigma}(y,[v])$ is well-defined for any nonzero $\sigma$ with sufficiently small $|\sigma|$. Furthermore, it only depends on the sign of $\sigma$.

Definition. For any $(y,[v]) \in \tilde{\mathcal{P}}$,

$$
\begin{cases}\text { ind }_{+}(y,[v]):=\tilde{\text { ind }}^{\sigma}(y,[v]) & \text { for a } \sigma \in \mathbb{R}^{-} \text {with }|\sigma| \ll 1 \\ \text { ind_- }_{-}(y,[v]):=\tilde{\text { ind }}^{\sigma}(y,[v]) & \text { for a } \sigma \in \mathbb{R}^{+} \text {with }|\sigma| \ll 1\end{cases}
$$

It is immediate from the definition that

$$
\tilde{\operatorname{ind}}_{+}(y,[v])-\tilde{\operatorname{ind}}_{-}(y,[v])=\operatorname{dim} \text { coker } A_{y},
$$

in particular, it is 0 if $y$ is non-degenerate; 1 if $y$ is minimally degenerate. 
It is also immediate that $\tilde{i n d} d_{+}, \tilde{i n d}_{-}$are, respectively, the upper semicontinuous and lower semicontinuous extension of the function ind on $\tilde{\mathcal{P}} \backslash \tilde{\mathcal{P}}$ deg . Thus, with the definitions

$$
\begin{aligned}
\operatorname{ind}_{ \pm} & :=\tilde{i n d} \bmod 2, \\
\operatorname{gr}_{+}((x,[w]),(y,[v])) & :=\tilde{\operatorname{ind}}_{+}(x,[w])-\tilde{\operatorname{ind}}_{-}(y,[v]), \\
\operatorname{gr}_{-}((x,[w]),(y,[v])) & :=\tilde{i n d}_{-}(x,[w])-\tilde{\operatorname{ind}}_{+}(y,[v]),
\end{aligned}
$$

(26) indeed follows from (RHFS1).

Next, given a small positive $\epsilon$, let

$$
U_{\epsilon} \subset L_{2}^{2}\left(y^{*} K\right)=T_{y} \mathcal{C}
$$

be an $\epsilon$-neighborhood of 0 . It corresponds to a small neighborhood of $y$ in $\mathcal{C}$ via the exponential map $\exp (y, \cdot)$. The vector field $\mathcal{V}_{X}$ on $\mathcal{C}$ pulls back to a vector field $\Xi$ on $U_{\epsilon}$ :

$$
\Xi(\xi)=A_{y}(\xi)+n_{y}(\xi)
$$

$n_{y}$ being the non-linear term. We leave the reader the easy task of finding the explicit formula for $n_{y}$, and limit ourselves to the following remark: $n_{y}$ is in general a function of both $\xi$ and $\dot{\xi}$, but when $J_{t}$ is integrable near $y(t)$, it depends on $\xi$ only. (A formula for $n_{y}$ in an analogous situation may be found in [10] Lemma 3.2. Note that in the second line of Floer's formula, $X$ and $Y$ should be interchanged. Moreover, since we consider perturbation by symplectic vector fields, there will be an additional term $\Theta_{N}, \Theta(\theta, y, \xi):=\left(D_{3} e\right)^{-1} \check{\theta}_{X}(\theta, \exp (y, \xi))$ in the notation of $\left.[\mathbf{1 0}]\right)$.

We now state the conditions of the Proposition.

5.1.2. The conditions. Let $(x,[w]),(y,[v]) \in \tilde{\mathcal{P}}(X)$ be either nondegenerate or minimally degenerate, with $\operatorname{gr}_{+}((x,[w]),(y,[v]))=i \leq 1$. We want to describe the asymptotic behavior of any $u \in \mathcal{M}_{P}((x,[w]),(y,[v]))$ when one of $x, y$ is minimally degenerate. (The non-degenerate case is well-known.) Without loss of generality, suppose $y$ is minimally degenerate; the case when $x$ is minimally degenerate is completely analogous. Assume additionally the following:

(1) $J \in \mathcal{J}$ is such that $\forall t \in S^{1}, J_{t}$ is integrable in a small neighborhood of $y(t) \in M$.

(2) Let $\mathbf{e}_{y}$ be a unit vector in ker $A_{y}$. Then $\Pi_{\text {ker } A_{y}} \nabla_{\mathbf{e}_{y}} \nabla_{\mathbf{e}_{y}} n_{y}(0) \neq 0$. In fact, we shall choose $\mathbf{e}_{y}$ to be the unique unit vector such that

$$
\Pi_{\mathrm{ker} A_{y}} \nabla_{\mathbf{e}_{y}} \nabla_{\mathbf{e}_{y}} n_{y}(0)=C_{y} \mathbf{e}_{y} \quad \text { for a real } C_{y}>0 .
$$

(3) For any sufficiently small $\sigma>0, \mathcal{M}_{P}^{(\sigma,-\sigma)}((x,[w]),(y,[v]))=\emptyset$.

Note that conditions (2) and (3) above are in fact generic: Condition (2) holds for Hamiltonian perturbations in an open dense set of $V_{\delta}^{2}(J, X)$. Condition (3) holds for a Baire set in $V_{\delta}^{k}(J, X), k>1$, by the weighted 
version of Proposition 3.2.2, since according to Lemma 3.2.3, the expected dimension of $\hat{\mathcal{M}}_{P}^{(\sigma,-\sigma)}((x,[w]),(y,[v]))$ is

$$
\operatorname{gr}_{+}((x,[w]),(y,[v]))-1<0 .
$$

We now state the main result in this subsection:

5.1.3. Proposition. Assuming Section 5.1.2, let $u(s, t)$ be any element in the moduli space $\mathcal{M}_{P}((x,[w],(y,[v]))$, and write

$$
u(s)=\exp (y, \mu(s))
$$

for large s. Then there exist constants $C_{i}, C_{i}^{\prime}, i=0,1,2$, such that:

$$
\begin{aligned}
C_{0}^{\prime} / s & \leq\|\mu(s)\|_{2,2, t} \leq C_{0} / s ; \\
C_{1}^{\prime} s^{-2} & \leq\left\|\partial_{s} \mu(s)\right\|_{2,1, t} \leq C_{1} s^{-2} ; \\
C_{2}^{\prime} s^{-3} & \leq\left\|\partial_{s}^{2} \mu(s)\right\|_{2, t} \leq C_{2} s^{-3} .
\end{aligned}
$$

In fact, the argument below also yields estimates for higher derivatives, but we shall only need them up to order 2 .

The rest of this subsection is devoted to the proof of this Proposition.

First, recall that the usual Gromov compactness (such as in the proof of [10] Theorem 1) implies:

5.1.4. Lemma. For any sufficiently small $\epsilon$, there is an $s_{0}=s_{0}(\epsilon) \gg 1$ such that

$$
\|\mu(s)\|_{2,2, t}+\left\|\partial_{s} \mu(s)\right\|_{2,1, t}+\left\|\partial_{s}^{2} \mu(s)\right\|_{2, t} \leq \epsilon \quad \text { for all } s \geq s_{0} .
$$

In fact, $\|\mu\|_{C_{\epsilon}\left(\left[s_{0}, \infty\right) \times S^{1}\right)}$ is small.

This enables us to focus on the neighborhood $U_{\epsilon}$.

5.1.5. Existence of center manifold. Let ker $A_{y}^{\perp}$ be the $L^{2}$-orthogonal complement of ker $A_{y} \subset L^{2}\left(y^{*} K\right)$.

Lemma. There is a smooth map

$$
\zeta: \operatorname{ker} A_{y} \cap U_{\epsilon} \rightarrow \operatorname{ker} A_{y}^{\perp} \cap U_{\epsilon},
$$

such that its graph is a 1-submanifold of $U_{\epsilon}$ tangent to $\operatorname{ker} A_{y}$ at 0 , and tangent to $\Xi$ elsewhere.

This lemma follows from [36] Theorem 5.1.1 and the smoothness of $J, X$, since $A_{y}$ is $L^{2}$-self adjoint with one-dimensional zero-eigenspace. The graph of $\zeta$ above is a "center manifold" of the flow generated by $\mathcal{V}_{X}$; It can be made unique by extending $\Xi$ linearly outside $U_{\epsilon} \subset L_{2}^{2}\left(y^{*} K\right)$. (See [36].) 
A simple example. Let $M=\mathbb{R}^{2}=\{(x, y) \mid x, y \in \mathbb{R}\}, \omega=d x \wedge d y$. Suppose $J$ and $H$ are both $t$-independent; $J=J_{0}$ is the standard complex structure, and $H_{\lambda}(t, x, y)=x^{2}+\lambda y^{2}+y^{3}$. For the Floer system generated by $\left(J, \chi_{H_{0}}\right)$, the center manifold is simply the $y$-axis, and the flow on the center manifold (to 0 ) is easily seen to decay as $C / s$ by a straightforward computation.

5.1.6. Estimates for flows on the center manifold. We now show that in general, the flow on the center manifold has the same decay behavior exhibited by the example.

To solve for the flow on the center manifold, it suffices to solve for the flow on its projection to $\operatorname{ker} A_{y}$.

Let $b: \mathbb{R} \rightarrow \operatorname{ker} A_{y}$. If $b(s)+\zeta(b(s))$ is a flow of $\Xi$, then $b(s)$ satisfies:

$$
\frac{\mathrm{db}}{d s}+\Pi_{\mathrm{ker} A_{y}} n_{y}(b+\zeta(b))=0,
$$

where $\Pi_{\text {ker } A_{y}}$ denotes the $L^{2}$-orthogonal projection from $L^{2}\left(y^{*} K\right)$ to ker $A_{y}$.

Lemma. Assuming (40) and (41), then any non-constant flow on the center manifold $b(s)+\zeta(b(s))$ satisfies the following estimate:

$$
\bar{C}_{k}^{\prime} / s^{k+1} \leq \| \partial_{s}^{k}\left(b(s)+\zeta(b(s)) \|_{2, t} \leq \bar{C}_{k} / s^{k+1}\right.
$$

for some positive constants $\bar{C}_{k}, \bar{C}_{k}^{\prime}, \quad k=0,1,2$.

Proof. First observe the useful fact that on ker $A_{y}$, all the Sobolev norms are commensurate. Let $b(s)=\beta(s) \mathbf{e}_{y}$ for an $\mathbb{R}$-valued function $\beta(s)$. We have the following key inequalities: for all sufficiently small $b$,

$$
C^{\prime} \beta^{2} \geq\left|\left\langle\mathbf{e}_{y}, n_{y}(b+\zeta(b))\right\rangle_{2, t}\right| \geq C \beta^{2} .
$$

The left inequality is straightforward from the form of $n_{y}$; the right inequality is also straightforward from the definitions of $n_{y}$ and $\zeta$ by (40).

By the right inequality in (43) and (42) we have

$$
\frac{d \beta}{d s} \leq-C \beta^{2} \quad \text { for a positive constant } C .
$$

Suppose $\beta$ is a non-trivial solution. Then $\beta$ is nowhere zero, since it satisfies an ODE. We may thus divide both sides of the above expression by $\beta^{2}$ and integrate over $s$, which gives $\beta(s) \leq 1 /(C s+B)$ if $\beta\left(s_{0}\right) \geq 0$, and if $\beta\left(s_{0}\right)<0, \beta(s)$ goes to $-\infty$ as $s$ increases, contradicting our assumption. Similarly, using the left inequality of $(43)$, we obtain $\beta(s) \geq 1 /\left(C^{\prime} s+B^{\prime}\right)$. $\left(B, B^{\prime}\right.$ are some constants.) These estimates together with the fact that $\zeta$ vanishes up to first order establish the $k=0$ case of the lemma.

The $k=1$ case of the lemma follows from (42) and (43). Taking the $s$-derivative of (42) and using the $k=0,1$ cases of the lemma and (40), we get the $k=2$ case of the lemma. 
5.1.7. Approximation of $u(s)$ to the center manifold. Next, we show that other flows to $y$ approach the center manifold exponentially.

Lemma. Let $u(s)=\exp (y, \mu(s))$ be a flow (i.e., a solution of (15)) to $y$ satisfying (41). Write

$$
\mu(s)=a(s)+c(s)
$$

where $a(s):=b(s)+\zeta(b(s))$ is a path on the center manifold, $b(s) \in \operatorname{ker} A_{y}$, and $c(s) \in \operatorname{ker} A_{y}^{\perp}$. Then there exists positive constants $C, \gamma$ such that

$$
\|c(s)\|_{2,2, t}+\left\|\partial_{s} c(s)\right\|_{2,1, t}+\left\|\partial_{s}^{2} c(s)\right\|_{2, t} \leq C e^{-\gamma s} \quad \forall s \geq s_{0} .
$$

Proof. Observe that since $a(s)$ is in the center manifold, the vector field $\Xi$ is parallel to $\partial_{s} a$. Thus, the flow equation (15) becomes:

$$
\begin{gathered}
-\frac{d a}{d s}=\left(1+\nabla_{b} \zeta\right) \Pi_{\mathrm{ker} A_{y}} n_{y}(a+c) ; \\
-\frac{d c}{d s}=A_{y} c+\left(1-\Pi_{\operatorname{ker} A_{y}}-\nabla_{b} \zeta \Pi_{\operatorname{ker} A_{y}}\right)\left(\left(n_{y}(a+c)-n_{y}(a)\right) .\right.
\end{gathered}
$$

Let's further decompose $c$ into

$$
c=c_{+}+c_{-},
$$

where $c_{+}$is in the completion of the direct sum of eigenspaces of $A_{y}$ with positive eigenvalues; similarly for $c_{-}$.

Note that the estimate

$$
\|c(s)\|_{2, t} \leq C e^{-\gamma s}
$$

follows from the routine arguments in [36] pp. 91-92 and the next lemma. The constant $\gamma$ is a positive number slightly smaller than the number $\nu_{-}$ below.

Sublemma. Assume Section 5.1.2. Let $\nu_{+}$be the minimal positive eigenvalue of $-A_{y}$, and let $-\nu_{-}$be the maximal negative eigenvalue. Then there exist positive constants $\epsilon_{ \pm}$, with $\epsilon_{ \pm} \ll \nu_{ \pm}$, such that the following hold:

$$
\begin{gathered}
\frac{d}{d s}\left\|c_{+}\right\|_{2, t} \geq \nu_{+}\left\|c_{+}\right\|_{2, t}-\epsilon_{+}\|c\|_{2, t} ; \\
\frac{d}{d s}\left\|c_{-}\right\|_{2, t} \leq-\nu_{-}\left\|c_{-}\right\|_{2, t}+\epsilon_{-}\|c\|_{2, t},
\end{gathered}
$$

Proof. The first inequality follows by taking the inner product of (45) with $c_{+}$, using the facts that $C^{1} \subset L_{2}^{2}$ in one-dimension, that $\|a\|_{2,2, t}+\|c\|_{2,2, t}$ is small, and that $n_{y}$ is a function of $\xi$ only under the assumption Section 5.1.2 (2.1).

The second inequality is similar.

To get estimates on higher derivatives of $c$, we perform elliptic bootstrapping in the following way. Re-arranging (45) and writing

$$
A_{y}=J_{t} \partial_{t}+\Upsilon_{y}
$$


we have

$$
\left(\partial_{s}+J_{t} \partial_{t}\right) c=-\Upsilon_{y} c+\left(1-\Pi_{\mathrm{ker} A_{y}}-\nabla_{b} \zeta \Pi_{\mathrm{ker} A_{y}}\right)\left(n_{y}(a+c)-n_{y}(a)\right) .
$$

Let $\beta_{\underline{s}, j}: \mathbb{R} \rightarrow \mathbb{R}$ be a smooth cutoff function that is supported on $(\underline{s}-2 j, \underline{s}+2 j)$, and is 1 over $[\underline{s}-j, \underline{s}+j]$. Let $\Theta_{\underline{s}, j}:=[\underline{s}-j, \underline{s}+j] \times S^{1}$. Multiplying the above expression by $\beta_{\underline{s}, 1}(s)$ and squaring both sides, we obtain

$$
\begin{aligned}
& \left\|\beta_{\underline{s}, 1} \partial_{s} c\right\|_{L^{2}\left(\Theta_{\underline{s}, 2}\right)}^{2}+\left\|\beta_{\underline{s}, 1} \partial_{t} c\right\|_{L^{2}\left(\Theta_{\underline{s}, 2}\right)}^{2} \\
& \quad \leq C\left\|\beta_{\underline{s}, 1} c\right\|_{L^{2}\left(\Theta_{\underline{s}, 2}\right)}^{2}+C^{\prime}\left\|\beta_{\underline{s}, 1}^{\prime} c\right\|_{L^{2}\left(\Theta_{\underline{s}, 2}\right)}\left\|\beta_{\underline{s}, 1} \partial_{t} c\right\|_{L^{2}\left(\Theta_{\underline{s}, 2}\right)} \\
& \quad \leq C^{\prime \prime}\|c\|_{L^{2}\left(\Theta_{\underline{s}, 2}\right)}^{2}+1 / 2\left\|\beta_{\underline{s}} \partial_{t} c\right\|_{L^{2}\left(\Theta_{\underline{s}, 2}\right)}^{2}
\end{aligned}
$$

by the triangle inequality. Rearranging, we get:

$$
\left\|\partial_{s} c\right\|_{L^{2}\left(\Theta_{\underline{s}, 1}\right)}^{2}+\left\|\partial_{t} c\right\|_{L^{2}\left(\Theta_{\underline{s}, 1}\right)}^{2} \leq C_{3}\|c\|_{L^{2}\left(\Theta_{\underline{s}, 2}\right)}^{2} \leq C_{4} e^{-2 \gamma \underline{s}}
$$

by integrating (46).

Now, we may iterate this argument, replacing (47) by $s$ - or $t$-derivatives of the equation, and using (41) repeatedly. The generalizaton of (4.26) boundes $\left\|\nabla^{k} c\right\|_{L^{2}\left(\Theta_{\underline{s}, 2^{1-k}}\right)}^{2}$ in terms of $\left\|\nabla^{j} c\right\|_{L^{2}\left(\Theta_{\underline{s}, 2^{1-j}}\right)}^{2}$ for $j=0,1, \ldots, k-1$, and hence by $C_{k}^{\prime} e^{-\overline{2} \gamma \underline{s}}$.

On the other hand, by Sobolev embedding

$$
\|\eta(\underline{s})\|_{2, t} \leq C_{\varepsilon}\|\eta\|_{L_{1}^{2}\left(\Theta_{\underline{s}, \varepsilon}\right)} ;
$$

so the above estimates of $\left\|\nabla^{k} c\right\|_{L^{2}\left(\Theta_{\underline{s}, \varepsilon}\right)}$ gives the other estimates in Lemma 5.1.7.

5.1.8. Approximation of $a(s)$ to a flow on the center manifold. In Lemma 5.1.7, we showed that the flow $u(s)$ approximates a path $a(s)$ in the center manifold. However, $a(s)$ might not be a flow itself. Nevertheless, the next lemma shows that $a(s)$ approximates the flow on the center manifold described in Lemma 5.1.6 exponentially fast.

Lemma. Let $a(s)$ be a path in the center manifold in $U_{\epsilon}$ satisfying

$$
\begin{aligned}
\| \partial_{s} a(s) & +A_{y}(a(s))+n_{y}(a(s))\left\|_{2,1, t}+\right\| \partial_{s}\left[\partial_{s} a(s)+A_{y}(a(s))\right. \\
& \left.+n_{y}(a(s))\right] \|_{2, t} \leq C e^{-\gamma s} \quad \text { for all } s \geq s_{0} .
\end{aligned}
$$

Then there is a unique flow $z(s)$ on the center manifold such that

$$
\begin{gathered}
\|z(s)-a(s)\|_{2,2, t}+\left\|\partial_{s}(z(s)-a(s))\right\|_{2,1, t}+\| \partial_{s}^{2}(z(s) \\
-a(s)) \|_{2, t} \leq C^{\prime} e^{-\gamma s} \quad \text { for all } s \geq s_{0} .
\end{gathered}
$$

Proof. The estimate for $\|z(s)-a(s)\|_{2, t}$ is proved in [36] Lemma 5.3 .1 by a simple contraction mapping theorem argument. In fact, it is proved there that $\left\|\Pi_{\text {ker } A_{y}}(z(s)-a(s))\right\|_{2, t} \leq C_{1} e^{-\gamma s}$. However, as we noted before, since on the one-dimensional space ker $A_{y}$ the Sobolev norms are commensurate, 
this implies $\left\|\Pi_{\text {ker } A_{y}}(z(s)-a(s))\right\|_{2,2, t} \leq C_{2} e^{-\gamma s}$, which in turn implies the exponential decay of $\|z(s)-a(s)\|_{2,2, t}$ by the uniform bounds on $\nabla \zeta$ in $U_{\epsilon}$. Now the estimates for $\left\|\partial_{s}(z(s)-a(s))\right\|_{2,1, t},\left\|\partial_{s}^{2}(z(s)-a(s))\right\|_{2, t}$ can be derived from this iteratively via the condition (5.11) and the fact that $\partial_{s} z(s)+A_{y} z(s)+n_{y}(z(s))=0$.

5.1.9. Concluding the proof of Proposition 5.1.3. Following the notation of Lemma 5.1.7, if $\exp (y, a(s)+c(s))$ is a flow to $y$, then $a(s)$ satisfies (5.11) by the estimates on $c(s)$ in Lemma 5.1.7. So we may apply Lemma 5.1.8. On the other hand, the flow $z(s)$ obtained in Lemma 5.1 .8 cannot be constant, because otherwise by Lemmas 5.1.7 and 5.1.8, $u(s)$ approaches $y$ exponentially. This would then contradict the assumption Section 5.1.2 (3). Proposition 5.1.3 now follows from the combination of Lemmas 5.1.6, 5.1.7, and 5.1.8.

5.2. Fredholm theory and structure theorem for $\mathcal{M}_{P}(x, y)$. In this subsection we prove the structure theorem for moduli spaces $\mathcal{M}_{P}(x, y)$ when one or both of $x, y$ are minimally degenerate, analogous to the standard Proposition 3.2.2. (See Proposition 5.2.6 below).

Without loss of generality we again assume that $y$ is minimally degenerate.

Because of the degeneracy of $y$, the usual $L^{p}$ Fredholm theory fails in this situation. One does have a good Fredholm theory by working with the exponentially weighted Sobolev spaces introduced in Section 3.2.3: in particular, Lemma 3.2.3 works in this situation for a non-zero weight $\sigma_{2} \neq 0$. However, these Fredholm theories are unsuitable for the purpose of describing $\mathcal{M}_{P}(x, y)$ for the following reasons: Let $\sigma \in \mathbb{R}$ with $|\sigma| \ll 1$. If $\sigma>0$, one may work with the $L_{k:(\sigma,-\sigma)}^{p}$ norms to describe the moduli space $\mathcal{M}_{P}^{(\sigma,-\sigma)}(x, y)=\mathcal{M}_{P} \cap \mathcal{B}_{P}^{(\sigma,-\sigma)}(x, y)$. However, $\mathcal{M}_{P}^{(\sigma,-\sigma)}(x, y)$ does not contain the whole $\mathcal{M}_{P}(x, y)$, since the former contains only exponentially decaying flows, while the latter contains flows that are polynomially decaying, as seen in Section 5.1. On the other hand, one cannot take $\sigma<0$ either, because $L_{k:(\sigma,-\sigma)}^{p}$ would be too large for an appropriate local description of the moduli space: for example, the Kuranishi map would not be suitably bounded with respect to these norms (i.e., estimates for non-linear terms of the type of (II.3) fail).

The decay estimates in Section 5.1 suggest that polynomially weighted spaces would be the natural alternative. Fredholm theories of such spaces is relatively uncommon in the literature, see however $[\mathbf{1 0}, \mathbf{2 8}]$ for similar theories. We shall follow [10] Section 4 closely.

5.2.1. LT-decomposition and polynomial weights. Let $u \in \mathcal{M}_{P}^{i}(x, y)$, and write $u(s)=\exp (y, \mu(s))$ as before. Assume without loss of generality that $x$ is non-degenerate and $y$ is minimally degenerate. 
We saw in last subsection that as $s \rightarrow \infty, \mu^{\prime}(s) /\left\|\mu^{\prime}(s)\right\|_{2, t}$ converges to the degenerate direction ker $A_{y}$. We shall call it the "longitudinal direction". Given an section of $u^{*} K, \xi$, we denote its longitudinal and transversal component by $\xi_{\mathrm{L}}$ and $\xi_{\mathrm{T}}$, respectively. Namely,

$$
\xi=\xi_{\mathrm{T}}+\xi_{\mathrm{L}}
$$

where

$$
\xi_{\mathrm{L}}(s):=\left\|u^{\prime}(s)\right\|_{2, t}^{-2}\left\langle u^{\prime}(s), \xi(s)\right\rangle_{2, t} u^{\prime}(s) .
$$

Roughly speaking, in the transversal direction, things go the same way as in the non-degenerate case, while in the longitudinal direction novelties arise. Let $\Pi_{\mathrm{L}}, \Pi_{\mathrm{T}}$ denote the $L^{2}$-orthogonal projection to the longitudinal/ transversal directions, respectively.

Define the weight

$$
\sigma_{u}(s)= \begin{cases}\left\|u^{\prime}(0)\right\|_{2, t}^{-1} & \text { for } s \leq 0 . \\ \left\|u^{\prime}(s)\right\|_{2, t}^{-1} & \text { for } s \geq 0 .\end{cases}
$$

From the previous subsection we know that $C^{\prime} / s^{2} \geq \sigma_{u} \geq C / s^{2}$ for large $s$.

Definition. Let $u \in \mathcal{M}_{P}^{i}(x, y)$ as above. We define the following norms on $C_{0}^{\infty}\left(u^{*} K\right)$ :

$$
\begin{aligned}
\|\xi\|_{W_{u}} & :=\left\|\sigma_{u}^{1 / 2} \xi\right\|_{p, 1}+\left\|\sigma_{u} \xi_{\mathrm{L}}^{\prime}\right\|_{p} ; \\
\|\xi\|_{L_{u}} & :=\left\|\sigma_{u}^{1 / 2} \xi\right\|_{p}+\left\|\sigma_{u} \xi_{\mathrm{L}}\right\|_{p} .
\end{aligned}
$$

Let $W_{u}=W_{u}\left(u^{*} K\right), L_{u}=L_{u}\left(u^{*} K\right)$ denote the completion of $C_{0}^{\infty}$ with respect to the above two norms, respectively.

5.2.2. Proposition. (Fredholmness) Let $u \in \mathcal{N}_{P}^{i}(x, y)$, where $i \leq 1$, $y$ is minimally degenerate, and $x$ is non-degenerate. Then the operator $E_{u}: W_{u} \rightarrow L_{u}$ is bounded and Fredholm of index $i$.

With the obvious modification of the defnition of $W_{u}$ and $L_{u}$, the statement of this Proposition also holds when $x$ is minimally degenerate, and $y$ is non-degenerate or minimally degenerate.

Notation. In the case when at least one of the two end critical points is minimally degenerate, we shall always reserve the notation $E_{u}$ for the deformation operator between $W_{u}$ and $L_{u}$. The deformation operator between exponentially weighted Sobolev spaces will come with a superscript, of the form $E_{u}^{\left(\sigma_{1}, \sigma_{2}\right)}$ (cf. Section 3.2.3).

The proof of this Proposition occupies Section 5.2.3-5.2.5 below. Since it is not very different from the proof of $[\mathbf{1 0}]$ Theorem $4 \mathrm{a}$, we shall only provide details where modification or clarification is needed. 
5.2.3. Boundedness of $E_{u}$. Via (50), $E_{u}$ decomposes into four components:

$$
E_{\mathrm{LT}}:=\Pi_{\mathrm{T}} E_{u} \Pi_{\mathrm{L}},
$$

and similarly $E_{\mathrm{TT}}, E_{\mathrm{TL}}, E_{\mathrm{LL}}$. The boundedness is straightforward to check by looking at each of these components: The boundedness of $E_{\mathrm{TT}}$ is the same as the usual (non-degenerate) case; the boundedness of $E_{\mathrm{LT}}, E_{\mathrm{TL}}, E_{\mathrm{LL}}$ follows, respectively, from the observations (5.14), (5.16), (54) below.

5.2.4. Fredholmness of $E_{u}$. Simple linear algebra shows that if the diagonal components $E_{\mathrm{TT}}, E_{\mathrm{LL}}$ are Fredholm, and one of the off-diagonal components vanishes while the other is bounded, then $E_{u}$ is Fredholm and

$$
\text { ind } E_{u}=\text { ind } E_{\mathrm{TT}}+\text { ind } E_{\mathrm{LL}} \text {. }
$$

Our task is thus to verify each of the above.

- $E_{L T}$ is zero:

This follows immediately from the fact that $E_{u} u^{\prime}=0$.

- $E_{T L}$ is bounded:

Following Floer's computation (4.18) of [10] (but replacing $J \partial_{t}$ there by $\left.A_{u(s)}\right)$, when $s$ is large

$$
\left(E_{u} \xi_{\mathrm{T}}\right)_{\mathrm{L}}(s)=-2 \sigma_{u}^{2}(s)\left\langle u_{\mathrm{T}}^{\prime \prime}(s), \xi_{\mathrm{T}}(s)\right\rangle_{2, t} u^{\prime}(s) .
$$

$\left\|u_{\mathrm{T}}^{\prime \prime}(s)\right\|_{2, t}$ is estimated as follows. Write $u(s)=\exp (y, \mu(s))$, where

$$
\mu(s)=b(s)+\zeta(b(s))+c(s)
$$

as in the previous subsection, we see that

$$
\begin{aligned}
\mu^{\prime} & =(1+\nabla \zeta) b^{\prime}+c^{\prime} \\
\mu^{\prime \prime} & =b^{\prime \prime}+\nabla_{b^{\prime \prime}} \zeta+\nabla_{b^{\prime}} \nabla_{b^{\prime}} \zeta+c^{\prime \prime} .
\end{aligned}
$$

We know from Lemma 5.1.7 that $\left\|c^{\prime}(s)\right\|_{2, t},\left\|c^{\prime \prime}(s)\right\|_{2, t}$ decays exponentially. Thus, modulo exponentially decaying terms, $\left\|u_{\mathrm{T}}^{\prime \prime}(s)\right\|_{2, t}$ is bounded by

$$
C\left\|b^{\prime}(s)\right\|_{2,1, t}^{2}+\tilde{C}\|b(s)\|_{2,1, t}\left\|b^{\prime \prime}(s)\right\|_{2, t} \leq C^{\prime} s^{-4} .
$$

Thus, for large $s$

$$
\begin{aligned}
\left\|\left(E_{u} \xi_{\mathrm{T}}\right)_{\mathrm{L}}\right\|_{\mathrm{L}_{u}} & =\left\|\sigma_{u}\left(E_{u} \xi_{\mathrm{T}}\right)_{\mathrm{L}}\right\|_{p} \\
& \leq 2\left\|\sigma_{u}^{2}\left\langle u^{\prime \prime}, \xi_{\mathrm{T}}\right\rangle_{2, t}(s)\right\|_{p} \\
& \leq C\|\| \xi_{\mathrm{T}}\left\|_{2, t}(s)\right\|_{p, s} \\
& \leq C^{\prime}\left\|\xi_{\mathrm{T}}\right\|_{p},
\end{aligned}
$$

where the subscripts $s$ and $t$ in the third line above indicate which variable the norm is respect to; and the final $L^{p}$ norm is with respect to both variables $s$ and $t$. In the last step we used the fact that on $S^{1}$ the $L^{2}$-norm is bounded by the $L^{p}$ norm. 
- $E_{T T}$ and $E_{L L}$ are Fredholm: By a typical excision argument (see e.g., $[\mathbf{1 0}])$, it suffices to consider only $\xi$ supported on $\{(s, t): s \in$ $(R, \infty)\}$ for a large $R$, and to replace $u$ by $v$, which is a small loop in $\mathcal{C}$ from $y$ to itself, such that $v$ agrees with $u$ for large $s$. We assume further that $\left\|v^{\prime}(s)\right\|_{2,1, t} \neq 0$ and that $v$ is symmetric about $s=0$ for simplicity.

The Fredholmness of $E_{\mathrm{TT}}$ is shown in [10] in a way similar to the non-degenerate case. To see the Fredholmness of $E_{\mathrm{LL}}$, write $\xi_{\mathrm{L}}=: f \sigma_{v} v^{\prime}$, where $f$ is an $\mathbb{R}$-valued function on $s$. Then $E_{\mathrm{LL}}$ is equivalent to the operator $\frac{d}{d s}+\sigma_{v}^{\prime} \sigma_{v}^{-1}$ with domain and range being the completion of $C_{0}^{\infty}(\mathbb{R})$ with respect to the norms $N_{1}, N_{2}$, respectively:

$$
\|f\|_{N_{1}}:=\left\|\sigma_{v}^{1 / 2} f\right\|_{p}+\left\|\sigma_{v} f^{\prime}\right\|_{p} ; \quad\|f\|_{N_{2}}:=\left\|\sigma_{v} f\right\|_{p} .
$$

Conjugating this operator by $\sigma_{v}$ makes it the operator $\frac{d}{d s}$ between the completion with respect to the $N$ norm and $L^{p}$, where

$$
\|g\|_{N}:=\left\|\sigma_{v}^{-1 / 2} g\right\|_{p}+\left\|g^{\prime}\right\|_{p}
$$

This operator $\frac{d}{d s}: N \rightarrow L^{p}$ is obviously bounded,

and has a one-dimensional kernel, namely, the space of constant functions. Let $N_{0} \subset N$ be the codimension 1-subspace

$$
N_{0}:=\{g(s) \mid g(s) \in N, \quad g(0)=0\} .
$$

We claim that the restriction $d /\left.d s\right|_{N_{0}}$ has a bounded inverse. This would then imply the Fredholmness of $d / d s: N \rightarrow L^{p}$, and hence $E_{\mathrm{LL}}$.

A natural candidate for its right-inverse is the integral:

$$
\left(\frac{d}{d s}\right)^{-1} q(s):=\int_{0}^{s} q(\tilde{s}) d \tilde{s} .
$$

To see that this is well-defined and bounded, notice the following estimate for compactly supported $L_{1, \text { loc }}^{p}$ functions on $(0, \infty)$ :

$$
\begin{aligned}
\left\|g^{\prime}\right\|_{p}+\left\|\sigma_{v}^{-1 / 2} g\right\|_{p} & \leq\left\|g^{\prime}\right\|_{p}+C\left\|s^{-1} g\right\|_{p} \\
& \leq\left\|s \frac{d}{d s}\left(s^{-1} g\right)\right\|_{p}+(C+1)\left\|s^{-1} g\right\|_{p} \\
& \leq C^{\prime}\left\|\left(s \frac{d}{d s}+1\right)\left(s^{-1} g\right)\right\|_{p} \\
& =C^{\prime}\left\|\frac{d}{d s} g\right\|_{p}
\end{aligned}
$$

The third step above follows from the fact that the integral kernel of the operator $s \frac{d}{d s}+1$ decays as $s^{-1}$ for large $s$. 
Let $g(s)=\chi_{R}(s) \tilde{g}(s)$, where $\tilde{g}(s)=\int_{0}^{s} q(\tilde{s}) d \tilde{s}$, and $\chi_{R}(s)=$ : $\chi(s / R), \chi$ being a smooth cutoff function supported on $(-2,2)$, with $\chi(s)=1$ for $s \in[-1,1]$. Then the above estimate (56) yields

$$
\begin{aligned}
& \left\|\chi_{R} \tilde{g}^{\prime}\right\|_{L^{p}\left(\mathbb{R}^{+}\right)}+\left\|\sigma_{v}^{-1 / 2} \chi_{R} \tilde{g}\right\|_{L^{p}\left(\mathbb{R}^{+}\right)} \\
& \quad \leq C^{\prime}\left\|\chi_{R} q\right\|_{L^{p}\left(\mathbb{R}^{+}\right)}+C^{\prime \prime} R^{-1}\left\|\chi^{\prime}(s / R) \tilde{g}(s)\right\|_{L^{p}\left(\mathbb{R}^{+}\right)} \\
& \quad \leq C^{\prime}\left\|\chi_{R} q\right\|_{L^{p}\left(\mathbb{R}^{+}\right)}+C_{2}\|q\|_{L^{p}([0,2 R])} .
\end{aligned}
$$

(cf. Lemma II.3.3.3(c) for the second step). Taking $R \rightarrow \infty$ and combining with the analogous estimates on the other half of the real line, we see that $\tilde{g} \in N_{0}$ when $q \in L^{p}$; in fact,

$$
\|\tilde{g}\|_{N} \leq C_{n}\|q\|_{p}
$$

Thus, the expression (4.33) does give a well-defined, bounded inverse of $d / d s: N_{0} \rightarrow L^{p}$.

We may now conclude that $E_{\mathrm{LL}}$ is Fredholm, and hence so is $E_{u}$.

5.2.5. Computation of the index. Let $\sigma$ be a small positive number. We claim that

$$
\text { ind } E_{u}=\text { ind } E_{u}^{(-\sigma, \sigma)} \text {. }
$$

The index computation of $E_{u}$ stated in Proposition 5.2.2 would then follow from Lemma 3.2.3.

Since $L_{1:(-\sigma, \sigma)}^{p}$ and $L_{(-\sigma, \sigma)}^{p}$ contain $W_{u}$ and $L_{u}$, respectively, it suffices to show that $\operatorname{ker}\left(E_{u}^{(-\sigma, \sigma)}\right) \subset W_{u}$, and coker $E_{u} \subset\left(L_{(-\sigma, \sigma)}^{p}\right)^{*}$. We shall only show the first, since the second is similar. This boils down to estimating the solution $\xi$ of $E_{u}^{(-\sigma, \sigma)} \xi=0$ where $s$ is large. If $\xi$ solves $E_{u}^{(-\sigma, \sigma)} \xi=0$, then

$$
\begin{gathered}
\left(E_{u}^{(-\sigma, \sigma)} \xi_{\mathrm{T}}\right)_{\mathrm{T}}=0 \\
\left(E_{u}^{(-\sigma, \sigma)} \xi_{\mathrm{L}}\right)_{\mathrm{L}}+\left(E_{u}^{(-\sigma, \sigma)} \xi_{\mathrm{T}}\right)_{\mathrm{L}}=0,
\end{gathered}
$$

since as noted before, $\left(E_{u}^{(-\sigma, \sigma)} \xi_{\mathrm{L}}\right)_{\mathrm{T}}=0$.

Using these, we estimate $\left\|\xi_{\mathrm{T}}(s)\right\|_{2, t}$ like (4.23) of [10] as follows.

$$
\begin{aligned}
& \frac{d^{2}}{d s^{2}}\left\langle\xi_{\mathrm{T}}, \xi_{\mathrm{T}}\right\rangle_{2, t} \\
& \quad=2 \frac{d}{d s}\left\langle\xi_{\mathrm{T}}^{\prime}, \xi_{\mathrm{T}}\right\rangle_{2, t} \\
& \quad=-2 \frac{d}{d s}\left\langle A_{u(s)} \xi_{\mathrm{T}}, \xi_{\mathrm{T}}\right\rangle_{2, t} \\
& \quad=-4\left\langle A_{u(s)} \xi_{\mathrm{T}}, \xi_{\mathrm{T}}^{\prime}\right\rangle_{2, t}-2\left\langle A_{u(s)}^{\prime} \xi_{\mathrm{T}}, \xi_{\mathrm{T}}\right\rangle_{2, t} \\
& \quad=4\left\|A_{u(s)} \xi_{\mathrm{T}}\right\|_{2, t}^{2}+4\left\langle\left(A_{u(s)} \xi_{\mathrm{T}}\right)_{\mathrm{L}},\left(E_{u}^{(-\sigma, \sigma)} \xi_{\mathrm{L}}\right)_{\mathrm{L}}\right\rangle_{2, t}-2\left\langle A_{u(s)}^{\prime} \xi_{\mathrm{T}}, \xi_{\mathrm{T}}\right\rangle_{2, t}
\end{aligned}
$$


When $s$ is large (so that $u(s)$ is close to $y$ ), the terms in the last line above can be estimated as follows. For the first term, we have the standard estimate:

$$
4\left\|A_{u(s)} \xi_{\mathrm{T}}\right\|_{2, t}^{2} \geq 4 \nu^{2}\left\|\xi_{\mathrm{T}}(s)\right\|_{2, t}^{2},
$$

where $\nu$ is a positive number slightly smaller than $\min \left(\left|\mu_{i}\right|\right)$, and $\mu_{i}$ are non-zero eigenvalues of $A_{y}$.

For the second term, use the fact that $\left(\xi_{\mathrm{T}}^{\prime}\right)_{\mathrm{L}}=-\sigma_{u}\left\langle u_{\mathrm{T}}^{\prime \prime}, \xi_{\mathrm{T}}\right\rangle_{2, t}$ (take the $s$-derivative of the equation $\left\langle u^{\prime}, \xi_{\mathrm{T}}\right\rangle_{2, t}=0$ to see this) and the estimates for $E_{\mathrm{TL}}$ and $\left\|u_{\mathrm{T}}^{\prime \prime}\right\|_{2, t}$ obtained earlier. We get:

$$
\begin{aligned}
& 4\left\langle\left(A_{u(s)} \xi_{\mathrm{T}}\right)_{\mathrm{L}},\left(E_{u}^{(-\sigma, \sigma)} \xi_{\mathrm{L}}\right)_{\mathrm{L}}\right\rangle_{2, t} \\
& \quad=-4\left\langle\left(E_{u}^{(-\sigma, \sigma)} \xi_{\mathrm{T}}\right)_{\mathrm{L}},\left(E_{u}^{(-\sigma, \sigma)} \xi_{\mathrm{T}}\right)_{\mathrm{L}}\right\rangle_{2, t}+4\left\langle\left(\xi_{\mathrm{T}}^{\prime}\right)_{\mathrm{L}},\left(E_{u}^{(-\sigma, \sigma)} \xi_{\mathrm{L}}\right)_{\mathrm{L}}\right\rangle_{2, t} \\
& \quad \geq-C \sigma_{u}^{-2}\left\|\xi_{\mathrm{T}}(s)\right\|_{2, t}^{2} .
\end{aligned}
$$

Under the assumption Section 5.1.2 (1), the last term can both be bounded below by

$$
\begin{aligned}
& -C\left\|u^{\prime}(s)\right\|_{\infty, 1, t}\left\|\xi_{\mathrm{T}}(s)\right\|_{2, t}^{2} \\
& \quad \geq-C^{\prime}\left\|u^{\prime}(s)\right\|_{2,2, t}\left\|\xi_{\mathrm{T}}(s)\right\|_{2, t}^{2} \\
& \quad \geq-C^{\prime \prime} s^{-2}\left\|\xi_{\mathrm{T}}(s)\right\|_{2, t}^{2} \quad \text { for large } s .
\end{aligned}
$$

In summary, letting $q(s):=\left\|\xi_{\mathrm{T}}(s)\right\|_{2, t}^{2}$, we have

$$
q^{\prime \prime}(s) \geq 4 \nu^{2} q(s) \quad \text { for all large enough } s .
$$

Now if $q^{\prime}>-2 \nu q$ at some $s_{0}$, then $q$ must grow faster than $e^{2} \nu s$ for large $s$. This is because that by comparison principle, $q$ is larger than the solution of $q_{0}^{\prime \prime}=4 \nu^{2} q_{0}$ with initial conditions $q_{0}^{(k)}\left(s_{0}\right)=q^{(k)}\left(s_{0}\right)$ for $k=0,1$. And when $q^{\prime}(0)>-2 \nu q(0)$ such a solution grows exponentially with exponent $2 \nu$ at infinity. However, such a $q$ cannot come from an $\xi \in L_{1:(-\sigma, \sigma)}^{p}$, since the weight $\sigma$ is chosen to be much smaller than $\min \left|\mu_{i}\right|$, and thus smaller than $\nu$. On the other hand, if $q^{\prime} \leq-2 \nu q$ for all sufficiently large $s$, by integration one easily sees that $q \leq C e^{-2 \nu s}$; namely $\left\|\xi_{\mathrm{T}}(s)\right\|_{2, t} \leq C^{\prime} e^{-\nu s}$. Since $\xi_{\mathrm{T}}^{\prime}=-A_{u} \xi_{\mathrm{T}}-\left(E_{u}^{(-\sigma, \sigma)} \xi_{\mathrm{T}}\right)_{\mathrm{L}}$ by $(57)$, we have a similar estimate for $\xi_{\mathrm{T}}^{\prime}(s)$, and therefore $\left\|\xi_{\mathrm{T}}\right\|_{W_{u}}<\infty$.

Next we estimate the $\xi_{\mathrm{L}}$ component. Writing $\xi_{\mathrm{L}}(s)=f(s) \sigma_{u}(s) u^{\prime}(s)$ again, it satisfies the equation:

$$
f^{\prime}(s)+\sigma_{u}^{\prime}(s) \sigma_{u}^{-1} f+\left\langle u^{\prime}, E_{u}^{(-\sigma, \sigma)} \xi_{\mathrm{T}}\right\rangle_{2, t} \sigma_{u}=0 .
$$


We have seen that $\left\|\left(E_{u}^{(-\sigma, \sigma)} \xi_{\mathrm{T}}(s)\right)_{\mathrm{L}}\right\|_{2, t} \leq C \sigma_{u}(s)^{-1}\left\|\xi_{\mathrm{T}}(s)\right\|_{2, t}$ decays exponentially. Thus

$$
\begin{aligned}
f(s) & =C \sigma_{u}^{-1}(s)+\text { exponentially decaying terms, } \\
\left|\sigma_{u} f^{\prime}(s)\right| & \leq\left|\sigma_{u}^{\prime}(s) f(s)\right|+\text { exponentially decaying terms. } \\
\left|\sigma_{u}^{\prime}(s)\right| & =\sigma_{u}^{3}(s)\left|\left\langle u^{\prime}(s), u^{\prime \prime}(s)\right\rangle_{2, t}\right| \leq \sigma_{u}^{2}(s)\left\|u^{\prime \prime}(s)\right\|_{2, t} \leq C \sigma_{u}(s) s^{-1} .
\end{aligned}
$$

Thus $\left\|\xi_{\mathrm{L}}\right\|_{W_{u}} \leq C\left\|\beta s^{-1}\right\|_{p}+\varepsilon$ is bounded. The proof of Proposition 5.2.2 is now complete.

5.2.6. Proposition. (Structure of $\mathcal{M}_{P}(x, y)$ ) Suppose $\mathcal{P}(X)$ consists of either non-degenerate or minimally degenerate critical points, and that $J$ satisfies Section 5.1.2 (1) near any minimally degenerate critical point. Let $\delta$ be an arbitrary small positive number, and either $k>2, X$ satisfies (40), or $k=2, X$ arbitrary. Then there is a Baire set $V_{\delta}^{k, \operatorname{Preg}}(J, X) \subset V_{\delta}^{k}(J, X)$, such that for any $H \in V_{\delta}^{k, \operatorname{Preg}}(J, X)$, (RHFS2d) holds for the Floer system generated by $\left(J, X+\chi_{H}\right)$ (regarded as a constant homotopy of Floer systems).

With the Fredholm theory established, this basically follows from the routine arguments in Section 3. Thus, we shall only very briefly comment on the proof.

Since we restrict the Hamiltonian perturbation to be within $V_{\delta}^{2}(J, X)$, the set of critical points $\mathcal{P}\left(X+\chi_{H}\right)$ is independent of $H$, so are the deformation operators $A_{x}$ for any $x \in \mathcal{P}\left(X+\chi_{H}\right)$. The definition of the norms $W_{u}, L_{u}$ given in Section 5.2.1 involves $u$, and hence depends on $H$; however, it is useful to observe that the spaces $W_{u}$ and $L_{u}$ have an alternative description which is independent of $H$, as follows.

Notation. Let $p, q \in \mathcal{C}$, and $q=\exp (p, \tilde{q})$, then $T_{p, q}:=D_{2} \exp (p, \tilde{q})$ denotes the isomorphism from $T_{p} \mathrm{e}$ to $T_{q} \mathrm{e}$, where $D_{i}$ denotes the differential with respect to the $i$-th variable.

The following Lemma follows from Proposition 5.1.3 by straightforward estimates.

Lemma. Let $x, y, u$ be as in Proposition 5.2.2. Then on $C_{0}^{\infty}\left(u^{*} K\right)$, the norms $W_{u}$ and $L_{u}$ are commensurate with the the norms $W_{y}, L_{y}$ below, respectively:

$$
\begin{aligned}
\|\xi\|_{W_{y}} & :=\left\|\sigma_{y}^{1 / 2} \xi\right\|_{p, 1}+\left\|\sigma_{y} \Pi_{\mathrm{T}_{\mathrm{y}, \mathrm{u}(\mathrm{s})} \mathbf{e}_{\mathrm{y}}} \xi^{\prime}(s)\right\|_{p} \\
\|\xi\|_{L_{y}} & :=\left\|\sigma_{y}^{1 / 2} \xi\right\|_{p}+\left\|\sigma_{y} \Pi_{\mathrm{T}_{\mathrm{y}, \mathrm{u}(\mathrm{s})} \mathbf{e}_{\mathrm{y}}} \xi\right\|_{p},
\end{aligned}
$$

where $\sigma_{y}(s)=s^{-2}$ for $s \geq 1$, and $\sigma_{y}=1$ otherwise.

As usual, $W_{y}\left(u^{*} K\right), L_{y}\left(u^{*} K\right)$ will denote the completion of $C_{0}^{\infty}\left(u^{*} K\right)$ with respect to these norms. The necessary modification on the definition in the case when $x$ is minimally degenerate, $y$ is non-degenerate or minimally 
degenerate will also be implied. We usually prefer to work with the original defintions of $W_{u}, L_{u}$ in Section 5.2.1, because they give simpler estimates due to the fact that $E_{u} u^{\prime}=0$. However, the alternative definition just given is more convenient for describing the configuration space: Let

$$
\begin{aligned}
& \mathcal{B}_{P}^{W}(x, y)= \\
& \left\{\begin{array}{ll}
u & \begin{array}{l}
u \in L_{1, l o c}^{p}\left(\Theta, p_{2}^{*} T_{f}\right), \\
u(s, \cdot)=\exp \left(y, \xi_{+}(s, \cdot)\right) \text { for some } \xi_{+} \in W_{y}\left(p_{2}^{*}\left(y^{*} K\right)\right) \text { if } s>\rho_{+}(u), \\
u(s, \cdot)=\exp \left(x, \xi_{-}(s, \cdot)\right) \text { for some } \xi_{-} \in L_{1}^{p}\left(p_{2}^{*}\left(x^{*} K\right)\right) \text { if } s<\rho_{-}(u)
\end{array}
\end{array}\right\},
\end{aligned}
$$

where $\rho_{+}, \rho_{-}, p_{2}$ are as in Definition 3.2.3. The usual arguments show that this is a Banach manifold, with open neighborhoods modeling on $T_{u} \mathcal{B}_{P}^{W}(x, y)=W_{u}$, and there is a Banach bundle over $\mathcal{B}_{P}^{W}(x, y)$ with fibers $L_{u}$. Thus, $\mathcal{M}_{P}^{i, W}(x, y):=\mathcal{M}_{P}^{i} \cap \mathcal{B}_{P}^{W}(x, y)$ is again described as the zero locus of a Fredholm section. Since we showed in Section 5.2.5 that coker $E_{u}=\operatorname{coker} E_{u}^{(-\sigma, \sigma)}$, an exponentially weighted version of the transversality arguments in Section 3 implies that $\mathcal{M}_{P}^{i, W}\left(x, y ; J, X+\chi_{H}\right)$ is non-degenerate for $H$ in a Baire set $U_{1} \subset V_{\delta}^{2}(J, X)$. On the other hand, as remarked before, the conditions Section 5.1.2 (2) and (3) hold for a Baire set, say $U_{2}$, in $V_{\delta}^{2}(J, X)$. The decay estimate of Proposition 5.1 .3 implies that for $H \in U_{2}, \mathcal{M}_{P}^{i, W}\left(x, y ; J, X+\chi_{H}\right)$ contains the whole $\mathcal{M}_{P}^{i}\left(x, y ; J, X+\chi_{H}\right)$. (Thus we are justified in dropping the superscript $W$ henceforth.) Let $V_{\delta}^{2, \text { reg }}(J, X ; x, y):=U_{1} \cap U_{2} . \quad V^{2 \text {,Preg }}(J, X)$ is the intersection of such $V_{\delta}^{2, \text { reg }}(J, X ; x, y)$ for all pairs of $x, y$.

The compactness of the moduli space is the consequence of Gromov compactness plus the decay estimate of Proposition 5.1.3.

5.3. Estimates for the new critical points $y_{+}, y_{-}$. Let $\left\{\left(J_{\lambda}, X_{\lambda}\right)\right\}_{\lambda \in \Lambda}$ be a path in $\mathcal{J}_{K} \times \mathcal{X}$ generating a CHFS, where $\Lambda=[-1,1]$. Let $y \in \mathcal{P}^{\Lambda, \text { deg }}$, with $\Pi_{\Lambda}(y)=\lambda_{0}$. We saw that if $y$ is a (Zariski) smooth point of $\mathcal{P}^{\Lambda}$, then it is a minimally degenerate element of $\mathcal{P}\left(X_{\lambda_{0}}\right)$. For explicit estimates near $y$, it is convenient to further assume that $y$ is standard in the following sense.

5.3.1. Definition. A minimally degenerate $y$ in $\mathcal{P}^{\Lambda, \text { deg }}$ is said to be in a standard $d-b$ neighborhood if the following hold:

(1a) Section 5.1.2 (2) holds, namely $\Pi_{\mathbf{e}_{y}}\left(\nabla_{\mathbf{e}_{y}} \nabla_{\mathbf{e}_{y}} n_{y}(0)\right)=C_{y} \mathbf{e}_{y}$ for $C_{y}>0$,

(1b) $\Pi_{\mathbf{e}_{y}}\left(\nabla_{\mathbf{e}_{y}} \nabla_{c} n_{y}(0)\right)=0$ for all $c \perp \mathbf{e}_{y}$,

(1c) $\Pi_{\mathbf{e}_{y}}\left(\nabla_{\mathbf{e}_{y}} \nabla_{\mathbf{e}_{y}} \nabla_{\mathbf{e}_{y}} n_{y}(0)\right)=0$.

(2a) $J_{\lambda}$ is constant in $\lambda$ in a small neighborhood of $\Lambda$ containg $\lambda_{0}$, and $J_{\lambda, t}$ is integrable in a small neighborhood of $y(t) \in M$ for all $t \in S^{1}$. (Namely Section 5.1.2 (1)).

(2b) At $\lambda=\lambda_{0}, \partial_{\lambda} \check{\theta}_{X_{\lambda}}(y)=C_{y}^{\prime} \mathbf{e}_{y}$ for a constant $C_{y}^{\prime} \neq 0$,

(2c) At $\lambda=\lambda_{0}, \Pi_{\mathbf{e}_{y}} \partial_{\lambda} \nabla_{v} \check{\theta}_{X_{\lambda}}(y)=0 \quad \forall v \in L_{t}^{2}\left(y^{*} K\right)$, 
(2d) $\partial_{\lambda} \check{\theta}_{X_{\lambda}}$ is supported in a neighborhood of $y$ away from all the other critical points.

This assumption will be particularly useful in the proofs of the gluing theorems discussed in Part II.

Assuming this, in this subsection we show that $y$ bifurcates into two nondegenerate critical points $y_{\lambda \pm}$, and give some essential estimates of these new critical points. These estimates will be useful for the gluing theorems in Part II.

5.3.2. Lemma. Let $y \in \mathcal{P}^{\Lambda, \operatorname{deg}}$ be in a standard $d$-b neighborhood. Then $y$ is a local extremum of $\Pi_{\Lambda}$ : it is a local maximum when the constant $C_{y}^{\prime}$ in Section 5.3.1 (2b) is positive, and a local minimum otherwise.

Furthermore, let $\lambda \in \Lambda$ be close to $\lambda_{0}:=\Pi_{\Lambda}(y)$, and $\lambda<\lambda_{0}$ when $C_{y}^{\prime}>0$; $\lambda>\lambda_{0}$ otherwise. Let

$$
y_{\lambda+}=\exp \left(y, \eta_{\lambda_{+}}\right), \quad y_{\lambda_{-}}=\exp \left(y, \eta_{\lambda_{-}}\right) \in \mathcal{P}_{\lambda}
$$

be the two points near $y \in \mathcal{C}$. Then they are both non-degenerate, of index $\operatorname{ind}_{+}(y)$ and ind $-(y)$, respectively. Moreover,

$$
C_{ \pm}\left|\lambda-\lambda_{0}\right|^{-1 / 2} \leq \pm\left\langle\mathbf{e}_{y}, \eta_{\lambda \mp}\right\rangle_{2, t} \leq C_{ \pm}^{\prime}\left|\lambda-\lambda_{0}\right|^{-1 / 2},
$$

where $C_{ \pm}, C_{ \pm}^{\prime}$ are positive constants. Furthermore, the eigenvalue of $A_{y_{\lambda \mp}}$ with minimal absolute value is bounded above and below by multiples of $\pm\left|\lambda-\lambda_{0}\right|^{1 / 2}$, respectively.

Proof. Without loss of generality, let $C_{y}^{\prime}>0$.

For each $\eta \in L_{t}^{2}\left(y^{*} K\right)$, write

$$
\eta_{\mathrm{L}}:=\Pi_{\mathrm{ker} A_{y}} \eta=: \underline{\eta}_{\mathrm{L}} \mathbf{e}_{y}, \quad \eta_{\mathrm{T}}:=\eta-\eta_{\mathrm{L}} .
$$

Using the assumption that $y$ is in a standard d-b neighborhood, the defining equation of critical points, $\mathcal{V}_{X}=0$, takes the following form in the local coordinates about $y$ :

$$
\begin{aligned}
A_{y} \eta_{\mathrm{T}} & +\left(1-\Pi_{\text {ker } A_{y}}\right) n_{y}\left(\eta_{\mathrm{L}}+\eta_{\mathrm{T}}, \eta_{\mathrm{L}}+\eta_{\mathrm{T}}\right) \\
& +O\left(\left|\lambda-\lambda_{0}\right|^{2}\right)+O\left(\left|\lambda-\lambda_{0}\right|\left(\left\|\eta_{\mathrm{L}}\right\|_{2,1, t}^{2}+\left\|\eta_{\mathrm{T}}\right\|_{2,1, t}^{2}\right)^{1 / 2}\right)=0 ; \\
C_{y} \underline{\eta}_{\mathrm{L}}^{2}+C_{y}^{\prime}\left(\lambda-\lambda_{0}\right)+\Pi_{\mathrm{ker} A_{y}} n_{y}\left(\eta_{\mathrm{T}}, \eta_{\mathrm{T}}\right)+O\left(\|\eta\|_{2,1, t}\left(\left\|\eta_{\mathrm{L}}\right\|_{2,1, t}+\left\|\eta_{\mathrm{T}}\right\|_{2,1, t}^{2}\right)\right) & +O\left(\left|\lambda-\lambda_{0}\right|^{2}\right)+O\left(\left|\lambda-\lambda_{0}\right|\left(\left\|\eta_{\mathrm{L}}\right\|_{2,1, t}^{2}+\left\|\eta_{\mathrm{T}}\right\|_{2,1, t}^{2}\right)\right)=0 .
\end{aligned}
$$

Notation. In this paper, $O(\cdot)$ or $o(\cdot)$ can be either a number, or a function of $t$ whose $L_{1, t}^{2}$ norm is of the order indicated. 
When $\left|\lambda-\lambda_{0}\right|$ is small, we can assume that $\left(\left\|\eta_{\mathrm{L}}\right\|_{2,1, t}^{2}+\left\|\eta_{\mathrm{T}}\right\|_{2,1, t}^{2}\right)^{1 / 2}$ is small, and thus the first equation above implies:

$$
\left\|\eta_{\mathrm{T}}\right\|_{2,1, t} \leq C_{1}\left(\left\|\eta_{\mathrm{L}}\right\|_{2,1, t}^{2}+\left|\lambda-\lambda_{0}\right|^{2}\right)
$$

Substituting this into the second equation in (60), we see that there are two solutions for $\eta_{\mathrm{L}}$, and for both

$$
C_{2}\left|\lambda-\lambda_{0}\right|^{1 / 2} \leq\left\|\eta_{\mathrm{L}}\right\|_{2,1, t} \leq C_{2}^{\prime}\left|\lambda-\lambda_{0}\right|^{1 / 2} .
$$

This then implies via (61) that

$$
\left\|\eta_{\mathrm{T}}\right\|_{2,1, t} \leq C_{3}\left|\lambda-\lambda_{0}\right|
$$

The solution with positive/negative value of $\eta_{\mathrm{L}}$ is denoted by $\eta_{\lambda_{-}}, \eta_{\lambda_{+}}$, respectively.

We now estimate the small eigenvalue of $A_{y_{\lambda_{ \pm}}}$. Let $\xi_{\lambda} \in L_{1, t}^{2}\left(y_{\lambda_{-}}^{*} K\right)$, and let $\left(\xi_{\lambda}\right)_{0} \in L_{1, t}^{2}\left(y^{*} K\right)$ be defined by

$$
\exp \left(y, \eta_{\lambda-}+\left(\xi_{\lambda}\right)_{0}\right)=\exp \left(y_{\lambda-}, \xi_{\lambda}\right) .
$$

Using the relation between $\xi_{\lambda}$ and $\left(\xi_{\lambda}\right)_{0}$, we see that the lowest order term of $A_{y_{\lambda-}}$ is conjugate to the linearization of the left hand side of (60) at $\eta_{\lambda_{-}}$. The latter has the form

$$
\left(\begin{array}{cc}
A_{y}+O\left(\left\|\left(\eta_{\lambda-}\right)_{\mathrm{L}}\right\|_{2,1, t}\right) & O\left(\left\|\left(\eta_{\lambda-}\right)_{\mathrm{T}}\right\|_{2,1, t}\right) \\
0 & 2 C_{y} \underline{\left(\eta_{\lambda-}\right)} \mathrm{L}+o\left(\left\|\left(\eta_{\lambda-}\right)_{\mathrm{L}}\right\|_{2,1, t}\right)
\end{array}\right)
$$

in terms of the decomposition $L_{t}^{2}\left(y^{*} K\right)=\left(\operatorname{ker} A_{y}\right)^{\perp} \oplus$ ker $A_{y}$. Using the estimates on $\left\|\left(\eta_{\lambda_{-}}\right)_{\mathrm{L}}\right\|_{2,1, t},\left\|\left(\eta_{\lambda_{-}}\right)_{T}\right\|_{2,1, t}$ above, we see that the smallest eigenvalue is bounded above and below by positive multiples of $\left|\lambda-\lambda_{0}\right|^{1 / 2}$. Similarly for $A_{y_{\lambda+}}$.

\section{Existence of admissible $(J, X)$-homotopies}

In this section, we show that given a path $\left\{X_{\lambda}\right\}_{\lambda \in \Lambda}$ as in the statement of Theorem 4.1.1, and an arbitrary path $\left\{J_{\lambda}\right\}_{\lambda \in \Lambda}$ connecting $J_{1}, J_{2}$, the path $\left\{J_{\lambda}, X_{\lambda}\right\}_{\lambda \in \Lambda}$ can be perturbed into an "admissible $(J, X)$-homotopy" fixing the end points. (See Definition 6.2.1, Proposition 6.2.2 below.)

6.1. Structure of parameterized moduli spaces. In this subsection we discuss structure theorems of moduli spaces parameterized by an interval, parallel to the results in Sections 3.2 and 3.3. Since they follow from routine modification of Section 3, we shall omit most of the proofs, except for some brief comments on the form of the relevant deformation operators and configuration spaces, which we shall need in Part II. 
6.1.1. Some terminologies. Let $\Lambda \subset \mathbb{R}$ be an interval. We shall use the notation $J^{\Lambda}$ to denote either a $C_{\epsilon}$ complex structure on the pull-back bundle $p_{2}^{*} K$, where $p_{2}: \Lambda \times T_{f} \rightarrow T_{f}$ is the projection, or the path of complex structures $\left\{J_{\lambda}\right\}_{\lambda \in \Lambda} \subset \partial_{K}$ this defines. Similarly, $X^{\Lambda}$ denotes either a section in $C_{\epsilon}\left(p_{2}^{*} K\right)$, or a path $\left\{X_{\lambda}\right\}_{\lambda \in \Lambda}$. We denote $X^{\Lambda}:=C_{\epsilon}\left(p_{2}^{*} K\right)$, and let $\mathcal{J}_{K}^{\Lambda}$ be the space of $C_{\epsilon}$ complex structures on $p_{2}^{*} K$.

A $(J, X)$-homotopy is an element $\left(J^{\Lambda}, X^{\Lambda}\right) \in \mathcal{J}_{K}^{\Lambda} \times X^{\Lambda}$, or equivalently, the path $\left\{\left(J_{\lambda}, X_{\lambda}\right)\right\}_{\lambda \in \Lambda}$ in $\mathcal{J}_{K} \times \mathcal{X}$ this defines. $\Lambda$ is said to be the parameter of this $(J, X)$-homotopy.

A $(J, X) \in \mathcal{J}_{K} \times X$ is said to belong to a $(J, X)$-homotopy $\left\{\left(J_{\lambda}, X_{\lambda}\right)\right\}_{\lambda \in \Lambda}$ if $(J, X)=\left(J_{\lambda}, X_{\lambda}\right)$ for some $\lambda \in \Lambda$. If $S \subset \Lambda$ is a sub-interval, the $(J, X)$ homotopy $\left\{\left(J_{\lambda}, X_{\lambda}\right)\right\}_{\lambda \in S}$ is said to be a sub-homotopy (over $\left.S\right)$ of the $(J, X)$ homotopy $\left\{\left(J_{\lambda}, X_{\lambda}\right)\right\}_{\lambda \in \Lambda}$.

Let $\Lambda \subset \mathbb{R}$ be an interval, and $N \subset \Lambda$ consists of finite points. Then

$$
\mathcal{H}^{\Lambda}:=C_{\epsilon}^{\infty}\left(\Lambda \times T_{f}\right), \quad \mathcal{H}_{N}^{\Lambda}:=\left\{H^{\Lambda} \mid H^{\Lambda} \in \mathcal{H}^{\Lambda}, \quad H_{\lambda}=0 \forall \lambda \in N\right\} .
$$

We shall often call an $H^{\Lambda} \in \mathcal{H}^{\Lambda}$ a Hamiltonian isotopy, since it corresponds to a path of Hamiltonians $\left\{H_{\lambda}\right\}_{\lambda \in \Lambda}$, and hence also a path of Hamiltonian sympletomorphisms. Let $\chi_{H^{\Lambda}} \in X^{\Lambda}$ denote the path of symplectic vector fields $\left\{\chi_{H_{\lambda}}\right\}_{\lambda \in \Lambda}$.

\subsubsection{The structure of $\mathcal{P}^{\Lambda}$.}

Definition. Let $J^{\Lambda} \in \partial_{K}^{\Lambda}$ be such that $J_{\lambda, t}$ is semipositive for all $\lambda, t$. (Such shall be called a semipositive path). An $X^{\Lambda} \in X^{\Lambda}$ is said to be $J^{\Lambda}$-nondegenerate if the following hold:

(a) $\mathcal{P}^{\Lambda}\left(X^{\Lambda}\right)$ is a (Zariski) smooth, compact 1-manifold;

(b) for any $x_{\lambda} \in \mathcal{P}^{\Lambda}\left(X^{\Lambda}\right), x_{\lambda}(t) \notin M_{0}\left(J_{\lambda, t}\right) \forall t$;

(c) The projection $\Pi_{\Lambda}: \mathcal{P}^{\Lambda}\left(X^{\Lambda}\right) \rightarrow \Lambda$ is smooth.

Proposition. Fix a semipositive path $J^{\Lambda}$, and an $X^{\Lambda} \in X^{\Lambda}$. Then there is a Baire set $\mathcal{H}^{\Lambda, \operatorname{ndg}}\left(J^{\Lambda}, X^{\Lambda}\right) \subset \mathcal{H}^{\Lambda}$ such that $X^{\Lambda}+\chi_{H^{\Lambda}}$ is $J^{\Lambda}$-non-degenerate for all $H^{\Lambda} \in \mathcal{H}^{\Lambda, n d g}\left(J^{\Lambda}, X^{\Lambda}\right)$.

Suppose in addition that $\left(J_{\lambda}, X_{\lambda}\right)$ are regular pairs $\forall \lambda \in \partial \Lambda$, then $\mathcal{H}_{\partial \Lambda}^{\Lambda, \operatorname{ndg}}\left(J^{\Lambda}, X^{\Lambda}\right):=\mathcal{H}^{\Lambda, \operatorname{ndg}}\left(J^{\Lambda}, X^{\Lambda}\right) \cap \mathcal{H}_{\partial \Lambda}^{\Lambda}$ is Baire in $\mathcal{H}_{\partial \Lambda}^{\Lambda}$.

Note that $\mathcal{H}^{\Lambda, \text { ndg }}$ is in fact open dense, by the compactness of $\mathcal{P} \Lambda$.

The deformation operator that describes the local structure of $\mathcal{P}^{\Lambda}$ is an extension of $A_{x}$ : Let $x_{\lambda} \in \mathcal{P} \Lambda$,

$$
\begin{gathered}
\hat{A}_{x_{\lambda}}: \mathbb{R} \oplus L_{1}^{p}\left(x_{\lambda}^{*} K\right) \rightarrow L^{p}\left(x_{\lambda}^{*} K\right), \\
\hat{A}_{x_{\lambda}}(\alpha, \xi)=A_{x_{\lambda}} \xi+\alpha \partial_{\lambda} \check{\theta}_{X_{\lambda}}\left(x_{\lambda}\right)+\alpha \partial_{\lambda} J_{\lambda}\left(\partial_{t} x_{\lambda}-X_{\lambda}\right) .
\end{gathered}
$$

Note that the last term vanishes when $x_{\lambda} \in \mathcal{P}\left(X_{\lambda}\right)$. 
Notation. When necessary, we shall insert $J, X$ or $J^{\Lambda}, X^{\Lambda}$ as superscripts to emphasize the dependence of the operators on them.

\subsubsection{The structure of $\mathcal{P}^{\Lambda, \operatorname{deg}}$.}

Proposition. Let $\left(J^{\Lambda}, X^{\Lambda}\right)$ be as in Proposition 6.1.2. Then there is a Baire set

$$
\mathcal{H}^{\Lambda, r g}\left(J^{\Lambda}, X^{\Lambda}\right) \subset \mathcal{H}^{\Lambda, \operatorname{ndg}}\left(J^{\Lambda}, X^{\Lambda}\right)
$$

such that $\mathcal{P}_{\operatorname{deg}}^{\Lambda}\left(X^{\Lambda}+\chi_{H^{\Lambda}}\right)$ consists of finitely many (Zariski) smooth points for any $H^{\Lambda} \in \mathcal{H}^{\Lambda, r g}\left(J^{\Lambda}, X^{\Lambda}\right)$.

Proof. Let

$$
\mathcal{P}^{\mathcal{H}^{\Lambda}}\left(X^{\Lambda}\right):=\bigcup_{H^{\Lambda} \in \mathcal{H}^{\Lambda}} \mathcal{P}^{\Lambda}\left(X^{\Lambda}+\chi_{H^{\Lambda}}\right) \subset \Lambda \times \mathcal{C} \times \mathcal{H}^{\Lambda}
$$

and let

$$
\operatorname{pr}: \mathcal{P}^{\mathcal{H}^{\Lambda}}\left(X^{\Lambda}\right) \rightarrow \mathcal{H}^{\Lambda}, \quad \tilde{\Pi}_{\Lambda}: \mathcal{P}^{\mathcal{H}^{\Lambda}}\left(X^{\Lambda}\right) \rightarrow \Lambda
$$

denote the projections.

We denote an element of $\mathcal{P H}^{\mathcal{H}^{\Lambda}}\left(X^{\Lambda}\right)$ by a pair $\left(x_{\lambda}, H^{\Lambda}\right)$, where $H^{\Lambda} \in \mathcal{H}^{\Lambda}$; $x_{\lambda} \in \mathcal{P}^{\Lambda}\left(X^{\Lambda}+\chi_{H^{\Lambda}}\right)$. An element in

$$
T_{\left(x_{\lambda}, H^{\Lambda}\right)} \mathcal{P}^{\mathcal{H}^{\Lambda}}\left(X^{\Lambda}\right) \subset T_{\lambda} \Lambda \times T_{x_{\lambda}} \mathcal{e} \times T_{H^{\Lambda}} \mathcal{H}^{\Lambda}
$$

shall be written as a triple $\left(\alpha, \xi, h^{\Lambda}\right)$, the three components belonging to the three factors in the RHS of (4.42) respectively.

The kernel of $\operatorname{pr}_{*}: T \mathcal{P H}^{\mathcal{H}^{\Lambda}}\left(X^{\Lambda}\right) \rightarrow T \mathcal{H}^{\Lambda}$, when restricted to

$$
\mathcal{P}^{\mathcal{H}^{\Lambda, \mathrm{ndg}}}\left(X^{\Lambda}\right):=\operatorname{pr}^{-1} \mathcal{H}^{\Lambda, \operatorname{ndg}}\left(J^{\Lambda}, X^{\Lambda}\right),
$$

has constant rank. Thus it defines a real line bundle $\mathcal{T}$,

$$
\Pi_{\mathcal{T}}: \mathcal{T} \rightarrow \mathcal{P}^{\mathcal{H}^{\Lambda, \mathrm{ndg}}}\left(X^{\Lambda}\right)
$$

so that for each $H^{\Lambda} \in \mathcal{H}^{\Lambda, \operatorname{ndg}}\left(J^{\Lambda}, X^{\Lambda}\right)$,

$$
\left.\mathcal{T}\right|_{\mathcal{P}^{\Lambda}\left(J^{\Lambda}, X^{\Lambda}+\chi_{H^{\Lambda}}\right)}=T \mathcal{P}^{\Lambda}\left(J^{\Lambda}, X^{\Lambda}+\chi_{H^{\Lambda}}\right) .
$$

Regarding $\mathcal{T}$ as a sub-bundle of $T \mathcal{P}^{\mathcal{H}^{\Lambda}}\left(X^{\Lambda}\right)$, the induced map $\tilde{\Pi}_{\Lambda *}: \mathcal{T} \rightarrow T \Lambda$ corresponds to a section $\mathfrak{s}$ of $\mathcal{T} \otimes \tilde{\Pi}_{\Lambda}^{*} T^{*} \Lambda$. The zero locus

$$
\mathfrak{s}^{-1}(0)=\bigcup_{H \in \mathcal{H}^{\Lambda, \text { ndg }}} \mathcal{P}^{\Lambda, \operatorname{deg}}\left(X^{\Lambda}+\chi_{H^{\Lambda}}\right)
$$

is the universal moduli of degenerate critical points. If $\mathfrak{s}$ is transversal to the zero section, then the claim of the Proposition follows from the usual argument via the Sard-Smale theorem. 
The transversality is verified as follows. Consider $\left(x_{\lambda}, H^{\Lambda}\right) \in \mathfrak{s}^{-1}(0) \subset$ $\mathcal{P}^{\mathcal{H}^{\Lambda}, \text { ndg }}\left(X^{\Lambda}\right)$, and let $\left(\beta, 0, h^{\Lambda}\right) \in T_{\left(x_{\lambda}, H^{\Lambda}\right)} \mathcal{P}^{\mathcal{H}^{\Lambda}}\left(X^{\Lambda}\right)$, where $\beta \neq 0$. It satisfies:

$$
\begin{gathered}
\beta \partial_{\lambda}\left(\check{\theta}_{X_{\lambda}}+\nabla H_{\lambda}\right)\left(x_{\lambda}\right)+\nabla h_{\lambda}\left(x_{\lambda}\right)=0 ; \\
\Pi_{\mathbf{e}_{x_{\lambda}}} \partial_{\lambda}\left(\check{\theta}_{X_{\lambda}}+\nabla H_{\lambda}\right)\left(x_{\lambda}\right) \neq 0 .
\end{gathered}
$$

To prove transversality, it suffices to show that for some $\left(\beta, 0, h^{\Lambda}\right)$ as above,

$$
\left(\hat{A}_{x_{\lambda}}^{J^{\Lambda}, X^{\Lambda}+\chi_{H^{\Lambda}}}\right)^{-1}\left(\delta_{\left(\beta, 0, h^{\Lambda}\right)} \hat{A}_{x_{\lambda}}^{J^{\Lambda}, X^{\Lambda}+\chi_{H^{\Lambda}}}\right)\left(0, \mathbf{e}_{x_{\lambda}}\right) \in \mathbb{R} \oplus L_{1}^{p}\left(x_{\lambda}^{*} K\right)
$$

has non-trivial $\mathbb{R}$-component. (The expression $\delta_{\left(\beta, \eta, h^{\Lambda}\right)} \hat{A}_{x_{\lambda}}^{J^{\Lambda}, X^{\Lambda}+\chi_{H^{\Lambda}}}$ above denotes the variation of $\hat{A}_{x_{\lambda}}^{J^{\Lambda}, X^{\Lambda}+\chi_{H^{\Lambda}}}$, regarded as an operator-valued function on $\mathcal{P}^{\mathcal{H}^{\Lambda}}\left(X^{\Lambda}\right)$.)

According to (65), this is equivalent to requiring

$$
\begin{aligned}
& \Pi_{\mathbf{e}_{x_{\lambda}}}\left(\delta_{\left(\beta, 0, h^{\Lambda}\right)} \hat{A}_{x_{\lambda}}^{J^{\Lambda}, X^{\Lambda}+\chi_{H^{\Lambda}}}\right)\left(0, \mathbf{e}_{x_{\lambda}}\right) \\
& \quad=\left\langle\mathbf{e}_{x_{\lambda}}, \nabla \mathbf{e}_{x_{\lambda}}\left(\nabla h_{\lambda}\left(x_{\lambda}\right)+\beta \partial_{\lambda} \check{\theta}_{X_{\lambda}}\left(x_{\lambda}\right)+\beta \partial_{\lambda} \nabla H_{\lambda}\left(x_{\lambda}\right)\right)\right\rangle_{2, t} \mathbf{e}_{x_{\lambda}} \\
& \quad \neq 0 .
\end{aligned}
$$

Notice that $\mathbf{e}_{x_{\lambda}}$ is nowhere vanishing, being a non-trivial solution of a linear ODE. Thus, one may easily choose a pair of $\beta, h^{\Lambda}$ satisfying (66). Transversality is now verified.

To verify the compactness, suppose the opposite, that there are infinitely many degenerate critical points of $\mathcal{P}^{\Lambda, \operatorname{deg}}\left(J^{\Lambda}, X^{\Lambda}+\chi_{H^{\Lambda}}\right)$ for an $H^{\Lambda} \in$ $\mathcal{H}^{\Lambda, r g}\left(J^{\Lambda}, X^{\Lambda}\right)$. Since $\mathcal{P}^{\Lambda, \operatorname{deg}}\left(J^{\Lambda}, X^{\Lambda}+\chi_{H^{\Lambda}}\right)$ lies in the compact 1-manifold $\mathcal{P}^{\Lambda}\left(J^{\Lambda}, X^{\Lambda}+\chi_{H^{\Lambda}}\right)$, it must contain a subset accumulating at a point $x_{\lambda} \in$ $\mathcal{P}^{\Lambda}\left(J^{\Lambda}, X^{\Lambda}+\chi_{H^{\Lambda}}\right)$. This $x_{\lambda}$ cannot be in $\mathcal{P}^{\Lambda}\left(J^{\Lambda}, X^{\Lambda}+\chi_{H^{\Lambda}}\right) \backslash \mathcal{P}^{\Lambda, \operatorname{deg}}\left(J^{\Lambda}, X^{\Lambda}+\right.$ $\chi_{H^{\Lambda}}$ ), since non-degeneracy (as a critical point) is an open condition. Thus, $x_{\lambda}$ is an accumulation point in $\mathcal{P}^{\Lambda, \operatorname{deg}}\left(J^{\Lambda}, X^{\Lambda}+\chi_{H^{\Lambda}}\right)$. On the other hand, the assumption $H^{\Lambda} \in \mathcal{H}^{\Lambda, r g}$ means that $H^{\Lambda}$ is a regular value of the projection map pr $\left.\right|_{\mathfrak{s}^{-1}(0)}$, hence the linearization of $\left.\mathfrak{s}\right|_{\mathcal{P}^{\Lambda}\left(J^{\Lambda}, X^{\Lambda}+\chi_{H^{\Lambda}}\right)}$ at $x_{\lambda}$ must be surjective. This implies that $x_{\lambda}$ is an isolated point in $\mathcal{P} \Lambda$,deg $\left(J^{\Lambda}, X^{\Lambda}+\chi_{H^{\Lambda}}\right)$, and we have arrived at a contradiction.

\section{Remarks.}

(a) Notice that the linearization of $\left.\mathfrak{s}\right|_{\mathcal{P} \Lambda}$ corresponds to the second derivative of the projection map $\Pi_{\Lambda}: \mathcal{P}^{\Lambda} \rightarrow \Lambda$. Thus, a (Zariski) smooth point of $\mathcal{P}^{\Lambda, d e g}$ is a local extremum of $\Pi_{\Lambda}$. In another word, (RHFS1) holds for the CHFS generated by $\left(J^{\Lambda}, X^{\Lambda}+\chi_{H^{\Lambda}}\right)$, for any $H^{\Lambda} \in \mathcal{H}^{\Lambda, r g}\left(J^{\Lambda}, X^{\Lambda}\right)$. 
(b) A simple computation shows that $x_{\lambda}$ is a (Zariski) smooth point of $\mathcal{P}^{\Lambda, \mathrm{deg}}$ if it is in a standard d-b neighborhood.

6.1.4. The structure of $\mathcal{M}_{P}^{\Lambda}, \mathcal{M}_{O}^{\Lambda}$. Let $\left(J^{\Lambda}, X^{\Lambda}\right)$ be a $(J, X)$-homotopy such that $J^{\Lambda}$ is semipositive, and $X^{\Lambda}$ is $H^{1}$-codirectional. Furthermore, suppose (RHFS1) and (RHFS2d) hold for the CHFS associated to $\left(J^{\Lambda}, X^{\Lambda}\right)$. Our next goal is show that (RHFS2, 3) hold for generic Hamiltonian perturbations in the following set: Given integers $k, \kappa, k>1, \kappa \geq 0$, and a small positive real number $\delta$, let

$$
\begin{aligned}
& V_{\delta}^{\Lambda ; k}\left(J^{\Lambda}, X^{\Lambda}\right):=\left\{H^{\Lambda} \mid \begin{array}{c}
\left\|H^{\Lambda}\right\|_{C_{\epsilon}} \leq \delta ; \nabla^{i} H_{\lambda, t}\left(x_{\lambda}(t)\right)=0 \\
\forall x_{\lambda} \in \mathcal{P}\left(J_{\lambda}, X_{\lambda}\right), \lambda \in \Lambda, 0 \leq i \leq k
\end{array}\right\} ; \\
& V_{\delta}^{\Lambda ; k, \kappa}\left(J^{\Lambda}, X^{\Lambda}\right):=\left\{H^{\Lambda} \mid \begin{array}{c}
H^{\Lambda} \in V_{\delta}^{\Lambda ; k}\left(J^{\Lambda}, X^{\Lambda}\right),\left.\partial_{\lambda}^{j} \nabla^{i} H_{\lambda}\right|_{\lambda=\lambda_{0}}=0 \\
\forall \lambda_{0} \in \Lambda_{\mathrm{db}} 0 \leq i \leq k, 0 \leq j \leq \kappa
\end{array}\right\} ; \\
& V_{N, \delta}^{\Lambda ; k, \kappa}\left(J^{\Lambda}, X^{\Lambda}\right):=\left\{H^{\Lambda} \mid \begin{array}{c}
\left.H^{\Lambda} \in V_{\delta}^{\Lambda ; k, \kappa}\left(J^{\Lambda}, X^{\Lambda}\right), H_{\lambda}=0 \forall \lambda \in N\right\},
\end{array}\right.
\end{aligned}
$$

where $N \subset \Lambda$ consists of finitely many points.

Given a semi-positive path $J^{\Lambda}$, an element $u_{\lambda} \in \mathcal{M}_{P}^{\Lambda}$ or $\mathcal{M}_{O}^{\Lambda}$ is said to be $J^{\Lambda}$-regular if it is non-degenerate and satisfies:

$$
u_{\lambda}(\cdot, t) \cap M_{0}\left(J_{\lambda, t}\right)=\emptyset \forall t .
$$

A moduli space $\mathcal{M}_{P}^{\Lambda}$ or $\mathcal{M}_{O}^{\Lambda}$ is said to be $J^{\Lambda}$-regular if it consists of $J^{\Lambda}$-regular elements.

Proposition. Let $\left(J^{\Lambda}, X^{\Lambda}\right)$ be a $(J, X)$-homotopy such that $J^{\Lambda}$ is semipositive, and $X^{\Lambda}$ is $H^{1}$-codirectional. Suppose furthermore that $X^{\Lambda}$ is $J^{\Lambda}$ non-degenerate, and (RHFS1), (RHFS2d) hold for the CHFS generated by $\left(J^{\Lambda}, X^{\Lambda}\right)$. Let $k, \kappa, \delta$ be numbers specified before (67). Then there is a Baire set

$$
V_{\delta}^{\Lambda ; k, \kappa, \operatorname{reg}}\left(J^{\Lambda}, X^{\Lambda}\right) \subset V_{\delta}^{\Lambda ; k, \kappa}\left(J^{\Lambda}, X^{\Lambda}\right)
$$

such that for any $H^{\Lambda} \in V_{\delta}^{\Lambda ; k, \kappa, \mathrm{reg}}\left(J^{\Lambda}, X^{\Lambda}\right)$ and any subinterval $S \subset \Lambda$ :

(a) the properties (RHFS2, 3) hold for the CHFS associated to $\left(J^{\Lambda}, X^{\Lambda}+\right.$ $\left.\chi_{H^{\Lambda}}\right)$;

(b) for any integer $i \leq 1$, the parameterized moduli spaces $\hat{\mathcal{M}}_{P}^{S, i}\left(J^{\Lambda}, X^{\Lambda}+\right.$ $\left.\chi_{H^{\Lambda}}\right), \hat{\mathcal{M}}_{O}^{S, i, \operatorname{sim}}\left(J^{\Lambda}, X^{\Lambda}+\chi_{H^{\Lambda}}\right)$ are $J^{\Lambda}$-regular.

Suppose, in addition, that there is a subset $N \subset \Lambda$ of finitely many elements such that $\left(J_{\lambda}, X_{\lambda}\right)$ is a regular pair for any $\lambda \in N$. Then statements (a) and (b) above hold for $H^{\Lambda}$ in a Baire subset

$$
V_{N ; \delta}^{\Lambda ; k, \kappa, \mathrm{reg}}\left(J^{\Lambda}, X^{\Lambda}\right) \subset V_{N ; \delta}^{\Lambda ; k, \kappa}\left(J^{\Lambda}, X^{\Lambda}\right) .
$$


Notice that if $\left(J^{\Lambda}, X^{\Lambda}\right)$ satisfies the conditions of this proposition, then any of its sub-homotopy satisfies the same conditions.

A $(J, X)$-homotopy is said to be regular if it can be written in the form $\left(J^{\Lambda}, X^{\Lambda}+\chi_{H^{\Lambda}}\right)$, where $\left(J^{\Lambda}, X^{\Lambda}\right)$ satisfies the conditions of the above proposition, and $H^{\Lambda} \in V_{\delta}^{\Lambda ; k, \kappa, \text { reg }}\left(J^{\Lambda}, X^{\Lambda}\right)$. If we only require statements (a) and (b) in the Proposition to hold for weight-truncated versions of moduli spaces $\hat{\mathcal{M}}_{P}^{S, i}\left(J^{\Lambda}, X^{\Lambda}+\chi_{H^{\Lambda}} ; \mathrm{wt}_{-\mathrm{y}, \mathrm{Lf}} \leq \Re\right), \hat{\mathcal{M}}_{O}^{S, i}\left(J^{\Lambda}, X^{\Lambda}+\chi_{H^{\Lambda}} ; \mathrm{wt}-\mathrm{y}, \mathrm{Lf} \leq \Re\right)$, $\forall \Re<R$, then $\left(J^{\Lambda}, X^{\Lambda}+\chi_{H^{\Lambda}}\right)$ is said to be a $R$-regular ( $\left.J, X\right)$-homotopy.

\subsubsection{Configuration spaces and deformation operators: parame-} terized versions. We introduce here the configuration space $\mathcal{B}_{P}^{\Lambda}$ and the deformation operator $\hat{E}_{u_{\lambda}}$. These notions will be needed in Part II.

Let $\left(J^{\Lambda}, X^{\Lambda}\right), S$ be as in Proposition 6.1.4, and let $\mathbf{x}, \mathbf{y} \in \aleph_{\Lambda}$. The topology of $\mathcal{M}_{P}^{S,\left(\sigma_{1}, \sigma_{2}\right)}(\mathbf{x}, \mathbf{y})$ is given by its embedding into:

$$
\mathcal{B}_{P}^{S,\left(\sigma_{1}, \sigma_{2}\right)}(\mathbf{x}, \mathbf{y}):=\bigcup_{\lambda \in S \cap \Lambda_{\mathbf{x}} \cap \Lambda_{\mathbf{y}}} \mathcal{B}_{P}^{\left(\sigma_{1}, \sigma_{2}\right)}\left(x_{\lambda}, y_{\lambda}\right),
$$

which is a Banach manifold, according to the argument of [10] Theorem 3a.

As usual, we omit the superscript $\left(\sigma_{1}, \sigma_{2}\right)$ when it is $(0,0)$.

Notation. An element of $\mathcal{B}_{P}^{S}(\mathbf{x}, \mathbf{y})$ will be denoted either by $u_{\lambda}$, as before, or as a pair $(\lambda, u)$, where $\lambda \in S$ and $u \in \mathcal{B}_{P}\left(x_{\lambda}, y_{\lambda}\right)$. The second notation is better suited for the discussion that follows.

The local model of a neighborhood of $(\lambda, u) \in \mathcal{B}_{P}^{S,\left(\sigma_{1}, \sigma_{2}\right)}(\mathbf{x}, \mathbf{y})$ is

$$
T_{(\lambda, u)} \mathcal{B}_{P}^{S,\left(\sigma_{1}, \sigma_{2}\right)}(\mathbf{x}, \mathbf{y})=\mathbb{R} \times L_{1:\left(\sigma_{1}, \sigma_{2}\right)}^{p}\left(u^{*} K\right),
$$

given via the map sending $(\mu, \xi) \in \mathbb{R} \times L_{1:\left(\sigma_{1}, \sigma_{2}\right)}^{p}\left(u^{*} K\right)$ to $(\lambda+\mu, e(\lambda, u ; \mu, \xi))$ $\in \mathcal{B}_{P}^{S,\left(\sigma_{1}, \sigma_{2}\right)}(\mathbf{x}, \mathbf{y})$, where $e(\lambda, u ; \mu, \xi) \in \mathcal{B}_{P}^{\left(\sigma_{1}, \sigma_{2}\right)}\left(x_{\lambda+\mu}, y_{\lambda+\mu}\right)$ is:

$$
\begin{aligned}
e(\lambda, u ; \mu, \xi)(s) & =e_{R, R^{\prime}}(\lambda, u ; \mu, \xi)(s) \\
& :=\exp \left(u(s), \xi(s)+\beta(-R-s) \bar{x}_{\mu}^{\lambda, u}(s)+\beta\left(s-R^{\prime}\right) \bar{y}_{\mu}^{\lambda, u}(s)\right),
\end{aligned}
$$

$R, R^{\prime}$ being two large positive constants, and $\bar{x}_{\mu}^{\lambda, u}(s), \bar{y}_{\mu}^{\lambda, u}(s) \in T_{u(s)} \mathrm{C}$ are defined by

$$
\exp \left(u(s), \bar{x}_{\mu}^{\lambda, u}(s)\right)=x_{\lambda+\mu} ; \quad \exp \left(u(s), \bar{y}_{\mu}^{\lambda, u}(s)\right)=y_{\lambda+\mu} .
$$

Note that the manifold structure of $\mathcal{B}_{P}^{S,\left(\sigma_{1}, \sigma_{2}\right)}(\mathbf{x}, \mathbf{y})$ does not depend on the choice of $R, R^{\prime}$, though the local coordinates certainly do. For later applications, we often choose $R, R^{\prime}$ to depend on $u, \lambda$. (See e.g., II.2.2.2). 
There is a Banach bundle over $\mathcal{B}_{P}^{S,\left(\sigma_{1}, \sigma_{2}\right)}(\mathbf{x}, \mathbf{y})$, of which the parameterized moduli space $\mathcal{M}_{P}^{S,\left(\sigma_{1}, \sigma_{2}\right)}\left(\mathbf{x}, \mathbf{y} ; J^{\Lambda}, X^{\Lambda}\right)$ is the zero locus of the Fredholm section $\bar{\partial}_{J^{\Lambda}, X^{\Lambda}}$,

$$
\bar{\partial}_{J^{\Lambda}, X^{\Lambda}}(\lambda, u):=\bar{\partial}_{J_{\lambda}, X_{\lambda}} u
$$

The linearization of $\bar{\partial}_{J^{\Lambda}, X^{\Lambda}}$ with respect to the above local coordinates of $\mathcal{B}_{P}^{S}(\mathbf{x}, \mathbf{y})$ has the form:

$$
\hat{E}_{(\lambda, u)}(\alpha, \xi)=E_{u}^{J_{\lambda}, X_{\lambda}} \xi+\alpha Y_{(\lambda, u)},
$$

where $Y_{(\lambda, u)} \in L^{p}\left(u^{*} K\right)$ has the following properties:

- It is supported on $\left(-R-1, R^{\prime}+1\right) \times S^{1}, R, R^{\prime}$ being the real numbers in $(68)$.

- Over $\left(-R, R^{\prime}\right) \times S^{1}$, it agrees with $\partial_{\lambda} \check{\theta}_{X_{\lambda}}(u)+\partial_{\lambda} J_{\lambda}\left(\partial_{t} u-X_{\lambda}\right)$.

- The difference $Y_{(\lambda, u)}-\left(\partial_{\lambda} \check{\theta}_{X_{\lambda}}\left(x_{\lambda}\right)+\partial_{\lambda} J_{\lambda}\left(\partial_{t} x_{\lambda}-X_{\lambda}\right)\right)$ consists of terms supported on $\left(-R-1, R^{\prime}+1\right) \backslash\left(-R, R^{\prime}\right) \times S^{1}$, that either involves a product of a $i$-th order derivative of $\beta\left(s-R^{\prime}\right)$ and a $j$-th order derivative of $\bar{y}_{\alpha}^{\lambda, u}$, or in parallel, a product of a $i$-th order derivative of $\beta(-R-s)$ and a $j$-th order derivative of $\bar{x}_{\alpha}^{\lambda, u}, i, j$ being 0 or 1 .

We shall see later that for practical purposes, the difference described in item 3 above is usually ignorable. Finally, note that the form of $\hat{E}_{(\lambda, u)}$ depends on the choice of local coordinates, and hence on the numbers $R, R^{\prime}$.

6.2. From a CHFS to an admissible homotopy. The purpose of this subsection is to use the structure theorems in Section 6.1 to establish the existence of admissible $(J, X)$-homotopies. We first state the definition:

6.2.1. Definition. A $(J, X)$-homotopy $\left(J^{\Lambda}, X^{\Lambda}\right)$ is said to be admissible, if the following three conditions hold:

(1) $J^{\Lambda}$ is a semi-positive path in $\partial_{K}$, and $X^{\Lambda}$ is $H^{1}$-codirectional. Plus, for $\lambda \in \partial \Lambda,\left(J_{\lambda}, X_{\lambda}\right)$ is regular.

(2) All the properties (RHFS*) hold except for (RHFS2c, 3c, 4).

(3) Any $x_{\lambda} \in \mathcal{P}^{\Lambda, \operatorname{deg}}\left(J^{\Lambda}, X^{\Lambda}\right)$ lies in a standard d-b neighborhood.

6.2.2. Proposition. Let $M$ be $w^{+}$-monotone, and $\left(J_{1}, X_{1}\right),\left(J_{2}, X_{2}\right)$ be two regular pairs. Suppose there is a $H^{1}$-codirectional path $X_{(0)}^{\Lambda}, \Lambda=$ $[1,2]$, connecting $X_{1}, X_{2}$. Then there exists an admissible $(J, X)$-homotopy connecting $\left(J_{1}, X_{1}\right),\left(J_{2}, X_{2}\right)$.

Remark. The Morse-theoretic picture leads one to expect that a generic $(J, X)$-homotopy satisfying condition 1 of Definition 6.2 .1 always generates an RHFS. In particular, condition 3 of Definition 6.2.1 should be unnecessary. Since we only need the existence, not the genericity of RHFSs, there is no harm in imposing this condition to simplify the estimates in Part II.

The rest of this subsection is devoted to the proof. 
First, we give a linear ordering of the properties of an admissible $(J, X)$ homotopy. We shall find a sequence of $(J, X)$-homotopies, $\left\{\left(J_{(k)}^{\Lambda}, X_{(k)}^{\Lambda}\right)\right\}_{k=0}^{K}$, so that the $k$-th step $\left(J_{(k)}^{\Lambda}, X_{(k)}^{\Lambda}\right)$ satisfies Properties $(0)-(k)$. Thus, the last of the sequence will be an admissible $(J, X)$-homotopy.

\subsubsection{Ordering the properties of admissibility.}

(0) Definition 6.2.1, item 1. (Semi-positivity of $J^{\Lambda}$ and $H^{1}$-codirection of $X^{\Lambda}$.)

(1) (RHFS1). (Smoothness and compactness properties of $\mathcal{P}^{\Lambda}, \mathcal{P}^{\Lambda, \mathrm{deg}}$.)

(2) (RHFS1i). (Injectivity of $\left.\Pi_{\Lambda}\right|_{\mathcal{P} \Lambda, \text { deg }}$.)

(3) Condition (2a) of Definition 5.3.1 $\forall y \in \mathcal{P}^{\Lambda, \mathrm{deg}}$. (Integrability and $\lambda$-independence of $J^{\Lambda}$ near $\mathcal{P}^{\Lambda, \text { deg }}$.)

(4) Conditions (1a) $-(1 \mathrm{c})$ of Definition 5.3.1 $\forall y \in \mathcal{P}^{\Lambda, \mathrm{deg}}$. (Constraints on higher derivatives of $n_{y}$ for degenerate critical points.)

(5) (RHFS2d). (Smoothness and compactness properties of $\mathcal{M}_{P}\left(X_{\lambda}\right)$, for $\left.\lambda \in \Lambda_{\mathrm{db}}.\right)$

(6) Conditions (2b) $-(2 \mathrm{~d})$ of Definition 5.3.1 $\forall y \in \mathcal{P}^{\Lambda \text {,deg }}$. (Constraints on $\partial_{\lambda} \check{\theta}_{X_{\lambda}}$ and its derivatives for $\lambda \in \Lambda_{\mathrm{db}}$.)

(7) (RHFS2, 3). (Smoothness and compactness properties of $\mathcal{M}_{P}^{\Lambda}, \mathcal{M}_{O}^{\Lambda}$.)

(8) (RHFS2i). (Injectivity of $\left.\Pi_{\Lambda}\right|_{\hat{\mathcal{M}}_{P}^{\Lambda, 0}} \cdot$ )

To achieve these properties, we allow $J^{\Lambda}$ to vary among semi-positive paths, and $X^{\Lambda}$ to vary within the set $\left\{X_{(0)}^{\Lambda}+\chi_{H^{\Lambda}} \mid H^{\Lambda} \in \mathcal{H}^{\Lambda}\right\}$. Notice that such $X^{\Lambda}$ is $H^{1}$-codirectional if $X_{(0)}^{\Lambda}$ is. This ordering is chosen so that $J_{(k)}^{\Lambda}$, $\mathcal{P}_{(k)}^{\Lambda}$ are fixed from Step (3) on. (So that notions such as $\Lambda_{\mathrm{db}}$ is independent of $k$ for sufficiently large $k$, and we are free to suppress the superscript or parenthetical reference to $\left(J_{(k)}^{\Lambda}, X_{(k)}^{\Lambda}\right)$ from the notations in this case). The Hamiltonian perturbation $H^{\Lambda}$ is fixed step by step: The low order derivatives of $H_{\lambda}$ at $y_{\lambda}$ for $y_{\lambda} \in \mathcal{P}^{\Lambda, \text { deg }}$ are fixed in Step (4). The functions $H_{\lambda}$ for $\lambda \in \Lambda_{\mathrm{db}}$ are fixed in Step (5). The low order derivatives of $\partial_{\lambda} H_{\lambda}$ at $y_{\lambda} \forall y_{\lambda} \in \mathcal{P}^{\Lambda, \operatorname{deg}}$ are fixed in Step (6). Steps (7) and (8) use the remaining freedom of $H^{\Lambda}$.

6.2.4. Achieving properties (0)-(7). Step (0). By assumption, $X_{(0)}^{\Lambda}$ and $\left(J_{1}, X_{1}\right),\left(J_{2}, X_{2}\right)$ already satisfy the requirements of Property (0). To obtain the 0 -th $(J, X)$-homotopy, we just need to find a semipositive path $J_{(0)}^{\Lambda}$ connecting $J_{1}, J_{2}$.

This is easy under the assumption that $M$ is $w^{+}$-monotone: in this case, any two regular elements in $\partial_{K}$ can be connected by a semi-positive path. To see this, notice that $\mathcal{J}_{K}$ is path-connected, and by an analog of Lemma 3.1.2 (b), any path connecting $J_{1}, J_{2}$ may be perturbed into a "regular path", namely, a path $\left\{J_{\lambda}\right\}$ such that the space $\bigcup_{\lambda} S\left(A, J_{\lambda}\right) / G$ is a smooth manifold 
of expected dimension $\left(2 n+2 c_{1}(A)-4\right)$. On the other hand, the analog of Lemma 3.1.2 (a) says that regular paths are semi-positive.

Let $J_{(0)}^{\Lambda}$ be one such regular path; $\left(J_{(0)}^{\Lambda}, X_{(0)}^{\Lambda}\right)$ is our 0-th $(J, X)$-homotopy.

Step (1). Let $\left(J_{(1)}^{\Lambda}, X_{(1)}^{\Lambda}\right)=\left(J_{(0)}^{\Lambda}, X_{(0)}^{\Lambda}+\chi_{H_{(1)}^{\Lambda}}\right)$, where $H_{(1)}^{\Lambda}$ is in $\mathcal{H}^{\Lambda, r g}\left(J_{(0)}^{\Lambda}, X_{(0)}^{\Lambda}\right)$. By Propositions 6.1.2, 6.1.3, $\left(J_{(1)}^{\Lambda}, X_{(1)}^{\Lambda}\right)$ satisfies Properties (0) and (1).

Step (2). There might be two distinct $x, y \in \mathcal{P}_{\mathrm{deg}}^{\Lambda}\left(J_{(1)}^{\Lambda}, X_{(1)}^{\Lambda}\right)$ with $\Pi_{\Lambda} x=\Pi_{\Lambda} y$. However, a simple perturbation to $X_{(1)}^{\Lambda}$ may distinguish their values under the projection map $\Pi_{\Lambda}$. For example, one may vary $X_{(1)}^{\Lambda}$ locally near the image of $x$, setting $X_{(2)}^{\Lambda}$ so that

$$
X_{(2), \lambda}= \begin{cases}X_{(1), f(\lambda)} & \text { over } U_{x} \\ X_{(1), \lambda} & \text { outside } V_{x},\end{cases}
$$

where $U_{x} \subset V_{x}$ are two small regular neighborhoods of the image of the section $x: S^{1} \rightarrow T_{f}$, and $f: \Lambda \rightarrow \Lambda$ is a diffeomorphism that agrees with the identity outside a neighborhood of $\Pi_{\Lambda}(x)$, and that $f\left(\Pi_{\Lambda}(x)\right) \neq \Pi_{\Lambda}(x)$.

Notice that since $x$ and $y$ have disjoint image (being distinct solutions of a first-order ODE), $U_{x}, V_{x}$ may be chosen so that the image of $y$ lies outside of $V_{x}$. On the other hand, since the difference between $X_{(2), \lambda}$ and $X_{(1), \lambda}$ is supported on a contractible space, it is necessarily Hamiltonian. Thus, such $X_{(2)}^{\Lambda}$ is still $H^{1}$-codirectional. Now, $\left(J_{(1)}^{\Lambda}, X_{(2)}^{\Lambda}\right)$ might no longer satisfy Property (1), but one may add a further small Hamiltonian perturbation to regain Property (1), using again Propositions 6.1.2, 6.1.3. As long as the perturbation is sufficiently small, Property (2) is preserved. Setting $J_{(2)}^{\Lambda}=J_{(1)}^{\Lambda}$, we have obtained our 2 nd $(J, X)$-homotopy, $\left(J_{(2)}^{\Lambda}, X_{(2)}^{\Lambda}\right)$, satisfying Properties (0), (1), (2).

Step (3). For each $\lambda \in \Pi_{\Lambda} \mathcal{P}^{\Lambda, \operatorname{deg}}\left(X_{(2)}^{\Lambda}\right)$, we choose $J_{(3) ; \lambda}$ such that:

- $J_{(3) ; \lambda} \in \mathrm{J}_{K}^{\mathrm{reg}}$

- $\left.J_{(3) ; \lambda, t}\right|_{U_{y_{\lambda}} \cap \pi_{S}^{-1}\{t\}}$ is integrable $\forall t \in S^{1}$, where $y_{\lambda} \in \mathcal{P}\left(X_{\lambda}\right)$ is the unique degenerate critical point, $U_{y_{\lambda}}, V_{y_{\lambda}}$ are as in Step (2) above, and $\pi_{S}: T_{f} \rightarrow S^{1}$ is the projection.

- $J_{(3) ; \lambda}$ is close to $J_{(2) ; \lambda}$ in $C_{\epsilon}$-norm over $T_{f} \backslash V_{y_{\lambda}}$.

This is possible because of Lemma 3.1.2 (c). Moreover, arguing as in Step $(0)$, such $\left\{J_{(3) ; \lambda}\right\}_{\lambda \in \Lambda_{\mathrm{db}}}$ may be extended into a semi-positive (in fact, regular) path $J_{(3)}^{\Lambda}$. If one so desires, one may also take $J_{(3)}^{\Lambda}$ to $C_{\epsilon}$-approximate $J_{(2)}^{\Lambda}$, away from the image of all $x \in \mathcal{P}^{\Lambda, \operatorname{deg}}\left(X_{(2)}^{\Lambda}\right)$ in $\Lambda \times T_{f}$. By a reparametrization of $\Lambda$, one may assume that $J_{(3)}^{\Lambda}$ is constant in $\lambda$ in a 
small neighborhood of $\Pi_{\Lambda} \mathcal{P}^{\Lambda, \operatorname{deg}}\left(X_{(2)}^{\Lambda}\right) \subset \Lambda$. Noticing that reparametrizing $\Lambda$ preserves semipositivity, we now have a semipositive path $J_{(3)}^{\Lambda}$ satisfying Property (3).

The $(J, X)$-homotopy $\left(J_{(3)}^{\Lambda}, X_{(2)}^{\Lambda}\right)$ now satisfies Properties (0), (2), (3), but Property (1) may be lost. In this case, one again apply Propositions 6.1.2, 6.1.3 to find a $X_{(3)}^{\Lambda}=X_{(2)}^{\Lambda}+\chi_{H_{(3)}^{\Lambda}}$, so that $\left(J_{(3)}^{\Lambda}, X_{(3)}^{\Lambda}\right)$ satisfies Properties (0), (1), (3). If $H_{(3)}^{\Lambda}$ is sufficiently small, Property (2) will still be preserved.

Step (4). $\quad$ Property (4) may be obtained by simply setting $X_{(4)}^{\Lambda}=X_{(3)}^{\Lambda}$ $+\chi_{H_{(4)}^{\Lambda}}$, with $H_{(4)}^{\Lambda} \in V_{\partial \Lambda, \delta}^{\Lambda ; 2}\left(J_{(3)}^{\Lambda}, X_{(3)}^{\Lambda}\right)$, varying $\nabla^{2} \check{\theta}_{X_{\lambda}}\left(y_{\lambda}\right), \nabla^{3} \check{\theta}_{X_{\lambda}}\left(y_{\lambda}\right)$ for $y_{\lambda} \in \mathcal{P}^{\Lambda, \operatorname{deg}}\left(X_{(3)}^{\Lambda}\right)$, but leaving the lower order derivatives unchanged. Notice that the fact that $H_{(4)}^{\Lambda} \in V_{\partial \Lambda, \delta}^{\Lambda ; k}$ for $k>1$ implies that $\mathcal{P}^{\Lambda}\left(X_{(4)}^{\Lambda}\right)=\mathcal{P}^{\Lambda}\left(X_{(3)}^{\Lambda}\right)$ in $\Lambda \times \mathcal{C}$, and the deformation operators $A_{y_{\lambda}}, \hat{A}_{y_{\lambda}}$ also remain the same. Thus, Properties (0)-(3) are still valid for $\left(J_{(4)}^{\Lambda}, X_{(4)}^{\Lambda}\right):=\left(J_{(3)}^{\Lambda}, X_{(4)}^{\Lambda}\right)$.

Step (5). $\quad$ Let $\left(J_{(5)}^{\Lambda}, X_{(5)}^{\Lambda}\right)=\left(J_{(4)}^{\Lambda}, X_{(4)}^{\Lambda}+\chi_{H_{(5)}^{\Lambda}}\right)$, where $H_{(5)}^{\Lambda}$ is such that $H_{(5) ; \lambda} \in V_{\delta}^{k, \text { Preg }}\left(J_{(4) ; \lambda}, X_{(4), \lambda}\right), \forall \lambda \in \Lambda_{\mathrm{db}}$. By Proposition 5.2.6, Property (5) then holds for $\left(J_{(5)}^{\Lambda}, X_{(5)}^{\Lambda}\right)$. By taking $H_{(5)}^{\Lambda} \in V_{\partial \Lambda, \delta}^{\Lambda ; k, \kappa}\left(J_{(4)}^{\Lambda}, X_{(4)}^{\Lambda}\right)$ for $k>2$, Properties (0)-(4) remain valid.

Step (6). $\quad \operatorname{Set}\left(J_{(6)}^{\Lambda}, X_{(6)}^{\Lambda}\right)=\left(J_{(5)}^{\Lambda}, X_{(5)}^{\Lambda}+\chi_{H_{(6)}^{\Lambda}}\right)$, where $H_{(6)}^{\Lambda} \in V_{\partial \Lambda, \delta}^{\Lambda ; k, 0}\left(J_{(5)}^{\Lambda}, X_{(5)}^{\Lambda}\right)$ for $k>2$, choosing $\partial_{\lambda} \nabla H_{(6), \lambda}$ near the neighborhood of all $y_{\lambda} \in \mathcal{P}^{\Lambda, \operatorname{deg}}\left(J_{(5)}^{\Lambda}, X_{(5)}^{\Lambda}\right)$ so that Conditions (2b)-(2d) of Definition 5.3.1 are met. The smallness of $H_{(6)}^{\Lambda}$ and the constraints on its low order derivatives imply that Properties (0)-(5) remain valid.

Step (7). Set $\left(J_{(7)}^{\Lambda}, X_{(7)}^{\Lambda}\right)=\left(J_{(6)}^{\Lambda}, X_{(6)}^{\Lambda}+\chi_{H_{(7)}^{\Lambda}}\right)$, where $H_{(7)}^{\Lambda} \in V_{\partial \Lambda, \delta}^{\Lambda ; k, \kappa, \text { reg }}\left(J_{(6)}^{\Lambda}, X_{(6)}^{\Lambda}\right)$ for $k>2, \kappa \geq 1$. By Proposition 6.1.4, Property (7) holds for $\left(J_{(7)}^{\Lambda}, X_{(7)}^{\Lambda}\right)$; on the other hand, Properties (0)-(6) remain valid by the constraints on $k, \kappa$.

6.2.5. The final step: injectivity of $\left.\Pi_{\Lambda}\right|_{\hat{\mathcal{M}}_{P}^{\Lambda, 0}}$. More care is required for this last step, because unlike the case of Step (2), $\hat{\mathcal{M}}_{P}^{\Lambda, 0}$ consists of possibly infinitely many elements, while we are also under the extra constraints to preserve the many properties already established above.

To obtain Property (8), we shall again set $\left(J_{(8)}^{\Lambda}, X_{(8)}^{\Lambda}\right)=\left(J_{(7)}^{\Lambda}, X_{(7)}^{\Lambda}+\right.$ $\left.\chi_{H_{(8)}^{\Lambda}}\right)$, where $H_{(8)}^{\Lambda} \in V_{\partial \Lambda, \delta}^{\Lambda ; k, \kappa, \operatorname{reg}}\left(J_{(7)}^{\Lambda}, X_{(7)}^{\Lambda}\right)$ for $k>2, \kappa \geq 1$. The Hamiltonian perturbation $H_{(8)}^{\Lambda}$ will be chosen iteratively below, via a refinement of the standard transversality argument.

First, observe the following 
Lemma. Suppose Properties (0)-(7) above hold for the $(J, X)$-homotopy $\left(J^{\Lambda}, X^{\Lambda}\right)$, and let

$$
\left\{\left(\lambda_{1}, u_{1}\right),\left(\lambda_{2}, u_{2},\right), \ldots,\left(\lambda_{n}, u_{n}\right)\right\} \subset \hat{\mathcal{M}}_{P}^{\Lambda, 0}\left(J^{\Lambda}, X^{\Lambda}\right)
$$

be such that $\lambda_{1}, \lambda_{2}, \ldots, \lambda_{n-1} \in \Lambda$ are mutually distinct, and $\lambda_{n}$ agrees with one $\lambda_{i}$, for some $i \in\{1, \ldots, n-1\}$. Let $k, \kappa$ be as in Step (7). Then for any sufficiently small $\delta$, there exists an $H^{\Lambda} \in V_{\partial \Lambda, \delta}^{\Lambda, k, \kappa, \mathrm{reg}}\left(J^{\Lambda}, X^{\Lambda}\right)$, such that:

Denoting by $\left(\tilde{\lambda}_{j}, \tilde{u}_{j}\right)$ the unique element in $\hat{\mathcal{M}}_{P}^{\Lambda, 0}\left(J^{\Lambda}, X^{\Lambda}+\chi_{H^{\Lambda}}\right)$ close to $\left(\lambda_{j}, u_{j}\right)$ in $\mathcal{B}_{P}^{\Lambda}$, the points $\tilde{\lambda}_{1}, \tilde{\lambda}_{2}, \ldots, \tilde{\lambda}_{n-1}, \tilde{\lambda}_{n}$ are mutually distinct in $\Lambda$.

Proof. Ideally, we would like the Hamiltonian perturbation to:

(i) shift $\lambda_{n}$, but meanwhile

(ii) leave $\lambda_{1}, \ldots, \lambda_{n-1}$ unchanged.

To guarantee (ii), one may try an $h^{\Lambda} \in V_{\partial \Lambda, \delta}^{\Lambda, k, \kappa}\left(J^{\Lambda}, X^{\Lambda}\right)$, such that:

$h_{\lambda}$ vanishes when $\lambda \in \Lambda \backslash S_{\lambda_{n}}$, where $S_{\lambda_{n}} \subset \Lambda$ is a small interval about $\lambda_{n}$,

so that $S_{\lambda_{n}} \cap\left(\Lambda_{\mathrm{db}} \cup\left\{\lambda_{1}, \ldots, \lambda_{n-1}\right\}\right)=\emptyset ;$

(71) $h_{\lambda}$ is supported away from the image of $u_{i}$ in $T_{f}, \forall i \in\{1, \ldots, n-1\}$.

To explain how to achieve (i), some preliminary discussion is required. Let $\mathcal{B}_{P}^{\Lambda}=\coprod_{\mathbf{x}, \mathbf{y} \in \aleph_{\Lambda}} \mathcal{B}_{P}^{\Lambda}(\mathbf{x}, \mathbf{y})$, and

$$
\mathcal{M}_{P}^{1, V_{\partial \Lambda, \delta}^{\Lambda ; k, \kappa}}\left(J^{\Lambda}, X^{\Lambda}\right):=\bigcup_{H^{\Lambda} \in V_{\partial \Lambda, \delta}^{\Lambda ; k, \kappa}\left(J^{\Lambda}, X^{\Lambda}\right)} \mathcal{M}_{P}^{\Lambda, 1}\left(J^{\Lambda}, X^{\Lambda}+\chi_{H^{\Lambda}}\right) \subset \mathcal{B}_{P}^{\Lambda}
$$

be the universal moduli space. Let $(\lambda, u) \in \mathcal{M}_{P}^{\Lambda, 1}\left(J^{\Lambda}, X^{\Lambda}\right)$, and let $((\lambda, u), 0)$ denote the associated element in $\mathcal{M}_{P}^{1, V_{\partial \Lambda, \delta}^{\Lambda ; k, \kappa}}\left(J^{\Lambda}, X^{\Lambda}\right)$. An element in its tangent space

$$
\left(\alpha, \xi, h^{\Lambda}\right) \in \mathbb{R} \times L_{1}^{p}\left(u^{*} K\right) \times V_{\partial \Lambda, \delta}^{\Lambda ; k, \kappa}\left(J^{\Lambda}, X^{\Lambda}\right)=T_{((\lambda, u), 0)} \mathcal{M}_{P}^{1, V_{\partial \Lambda, \delta}^{\Lambda ; k, \kappa}}\left(J^{\Lambda}, X^{\Lambda}\right)
$$

satisfies

$$
E_{u}^{J_{\lambda}, X_{\lambda}}(\xi)+\alpha Y_{(\lambda, u)}+\nabla h_{\lambda}(u)=0
$$

$Y_{(\lambda, u)}$ being as in (69).

Let $\mathfrak{f}_{u}$ be a unit vector in coker $E_{u}$. Since $\left(J^{\Lambda}, X^{\Lambda}\right)$ is assumed to satisfy (RHFS2), $\left(\lambda_{j}, u_{j}\right)$ is an non-degenerate point of $\mathcal{M}_{P}^{\Lambda, 1}\left(J^{\Lambda}, X^{\Lambda}\right)$ for any $j$; hence coker $E_{u_{j}}=\operatorname{Span}\left\{\mathfrak{f}_{u_{j}}\right\}$ and

$$
\Pi_{\mathfrak{f}_{u_{j}}} Y_{(\lambda, u)} \neq 0 \text {. }
$$

Thus, by (72), a small $h^{\Lambda}$ would satisfy (i) if

$$
\Pi_{\mathfrak{f}_{u}} \nabla h_{\lambda}\left(u_{n}\right) \neq 0 \text {. }
$$


We now show the existence of $h^{\Lambda}$ satisfying all the three conditions (6.7), (6.8), (6.10).

As in the standard transversality argument (as in the proof of Theorem 5.1 (i) of [14]), consider the following three possibilities for $\mathfrak{f}_{u_{n}}$ :

Case 1: The set

$$
Q_{1}:=\left\{(s, t) \mid(s, t) \in \mathbb{R} \times S_{1}^{1}, \mathfrak{f}_{u_{n}}(s, t) \neq C u_{n}^{\prime}(s, t) \forall C \in \mathbb{R}\right\}
$$

is non-empty.

Case 2: $\mathfrak{f}_{u_{n}}=g(s, t) u_{n}^{\prime}$, and the set

$$
Q_{2}:=\left\{(s, t) \mid \partial_{s} g(s, t) \neq 0\right\}
$$

is non-empty.

Case 3: $\mathfrak{f}_{u_{n}}=g(t) u_{n}^{\prime}$.

Case 3 was shown to be impossible in the proof of Theorem 5.1 (i) of [14]. For the other two cases, apply unique continuation (cf. [14]) as in Section 3 to see that the image of $u_{i}, u_{n}$ at most intersect at discrete points. On the other hand, both sets $Q_{1}$ and $Q_{2}$ are open. Thus, in both cases 1 and 2, we can find a neighborhood $B$ in $Q_{1}$ or $Q_{2}$, such that $u_{n}(B)$ does not intersect the image of $u_{i}$.

In either case, we can choose a small $\underline{H}_{\lambda_{n}} \in V_{\delta}^{k}\left(J_{\lambda_{n}}, X_{\lambda_{n}}\right)$ supported in a small neighborhood $\mathfrak{B} \subset T_{f}$, so that $u_{n}^{-1}(\mathfrak{B}) \subset B$, and $\left\langle\mathfrak{f}_{u_{n}}, \nabla \underline{H}_{\lambda_{n}}\left(u_{n}\right)\right\rangle_{2} \neq$ 0, as in the proof of Theorem 5.1 (i) of [14]. This $\underline{H}_{\lambda_{n}}$ can be extended smoothly to get a small $\underline{H}^{\Lambda} \in V_{\partial \Lambda, \delta}^{\Lambda ; k, \kappa}\left(J^{\Lambda}, X^{\Lambda}\right)$ satisfying (6.7).

$\underline{H}^{\Lambda}$ now satisfies (6.7), (6.8), (6.10) by construction. Since $V_{\partial \Lambda, \delta}^{\Lambda, k, \kappa, \text { reg }}$ $\left(J^{\Lambda}, X^{\Lambda}\right)$ is dense in $V_{\partial \Lambda, \delta}^{\Lambda ; k, \kappa}\left(J^{\Lambda}, X^{\Lambda}\right)$, we can approximate $\underline{H}^{\Lambda}$ by an element $H^{\Lambda} \in V_{\partial \Lambda, \delta}^{\Lambda ; k, \kappa, \text { reg }}\left(J^{\Lambda}, X^{\Lambda}\right)$, still keeping $\Pi_{\Lambda}$ injective on $\left\{\left(\tilde{\lambda}_{1}, \tilde{u}_{1}\right), \ldots,\left(\tilde{\lambda}_{n-1}, \tilde{u}_{n-1}\right),\left(\tilde{\lambda}_{n}, \tilde{u}_{n}\right)\right\} \subset \hat{\mathcal{M}}_{P}^{\Lambda, 0}\left(J^{\Lambda}, X^{\Lambda}+\chi_{H^{\Lambda}}\right)$.

We now return to Step (8) of the proof of Proposition 6.2.2.

Since $\left(J_{(7)}^{\Lambda}, X_{(7)}^{\Lambda}\right)$ satisfies (RHFS2), there are countably many elements in $\hat{\mathcal{M}}_{P}^{\Lambda, 0}\left(J_{(7)}^{\Lambda}, X_{(7)}^{\Lambda}\right)$; let's enumerate them as $\left(\lambda_{1}, u_{1}\right),\left(\lambda_{2}, u_{2}\right), \ldots\left(\lambda_{n}, u_{n}\right), \ldots$, such that the weight $\mathrm{wt}_{-\langle y\rangle, e_{\mathcal{P}}}$ increases monotonically in $n$. An small Hamiltonian perturbation to $X_{(7)}^{\Lambda}$ will only change them slightly.

We now apply the previous Lemma recursively to the first $n$ elements of $\mathcal{M}_{P}^{\Lambda, 0}\left(J^{\Lambda}, X^{\Lambda}\right)$ (in the order of $\left.\mathrm{wt}_{-\langle y\rangle, e_{\mathcal{P}}}\right)$ for each $n$, labeling the $\left(J^{\Lambda}, X^{\Lambda}\right)$ used in the $n$-th step by $\left(\tilde{J}_{[n]}^{\Lambda}, \tilde{X}_{[n]}^{\Lambda}\right)$, the number $\delta$ used in the $n$-th step by $\tilde{\delta}_{[n]}$, and the small Hamiltonian pertubation $H^{\Lambda}$ obtained in the $n$-th step 
by $\tilde{H}_{[n]}^{\Lambda}$. Set

$$
\begin{gathered}
\tilde{J}_{[n]}^{\Lambda}=J_{(7)}^{\Lambda} \quad \forall n \in \mathbb{Z}^{+}, \\
\tilde{X}_{[1]}^{\Lambda}:=X_{(7)}^{\Lambda}, \quad \tilde{X}_{[n]}^{\Lambda}=\tilde{X}_{[n-1]}^{\Lambda}+\chi_{\tilde{H}_{[n-1]}^{\Lambda}} \quad \text { for } n>1 .
\end{gathered}
$$

Notice that

$$
V_{\partial \Lambda, \delta}^{\Lambda ; k, \kappa}\left(J^{\Lambda}, X^{\Lambda}\right)=V_{\partial \Lambda, \delta}^{\Lambda ; k, \kappa}\left(J^{\Lambda}, X^{\Lambda}+\chi_{H^{\Lambda}}\right) \quad \text { if } H^{\Lambda} \in V_{\partial \Lambda, \delta}^{\Lambda ; k, \kappa}\left(J^{\Lambda}, X^{\Lambda}\right) .
$$

Thus, all the various $\tilde{H}_{[n-1]}^{\Lambda}$, or any sum of them, are actually in the same space, namely $V_{\partial \Lambda, \delta}^{\Lambda ; k, \kappa}\left(J_{(7)}^{\Lambda}, X_{(7)}^{\Lambda}\right)$ for certain $\delta>0$.

To explain our choice of $\tilde{\delta}_{[n]}$, we need to first give an explicit description of the Baire set $V_{\partial \Lambda, \delta}^{\Lambda ; k, \kappa, \operatorname{reg}}\left(J^{\Lambda}, X^{\Lambda}\right)$ as a countable intersection of certain open dense sets.

Let $V_{\partial \Lambda, \delta}^{\Lambda ; k, \kappa, R \text {-reg }}\left(J^{\Lambda}, X^{\Lambda}\right) \subset V_{\partial \Lambda, \delta}^{\Lambda ; k, \kappa}\left(J^{\Lambda}, X^{\Lambda}\right)$ be the subset consisting of all $H^{\Lambda}$ such that $\left(J^{\Lambda}, X^{\Lambda}+\chi_{H^{\Lambda}}\right)$ is $R$-regular. By the compactness of truncated moduli spaces, this is an open dense set. By construction,

$$
V_{\partial \Lambda, \delta}^{\Lambda ; k, \kappa, \operatorname{reg}}\left(J^{\Lambda}, X^{\Lambda}\right)=\bigcap_{R>0} V_{\partial \Lambda, \delta}^{\Lambda ; k, \kappa, R \text {-reg }}\left(J^{\Lambda}, X^{\Lambda}\right) .
$$

When $\left(J^{\Lambda}, X^{\Lambda}\right)$ is regular, given $R \in \mathbb{R}^{+}$, there is an $\varepsilon_{R}\left(J^{\Lambda}, X^{\Lambda}\right) \in \mathbb{R}^{+}$such that

$$
V_{\partial \Lambda, \delta}^{\Lambda ; k, \kappa, R-\operatorname{reg}}\left(J^{\Lambda}, X^{\Lambda}\right)=V_{\partial \Lambda, \delta}^{\Lambda ; k, \kappa}\left(J^{\Lambda}, X^{\Lambda}\right) \quad \forall \delta \leq \varepsilon_{R}\left(J^{\Lambda}, X^{\Lambda}\right) .
$$

Now we are ready to state:

$$
\tilde{\delta}_{[n]}:=\min \left\{2^{-n+j-1} \varepsilon_{R_{j}}\left(\tilde{J}_{[j]}^{\Lambda}, \tilde{X}_{[j]}^{\Lambda}\right) \mid 1 \leq j \leq n\right\},
$$

where $R_{j}=\mathrm{wt}_{-\langle y\rangle, e_{\mathcal{P}}}\left(u_{j}\right)$. Since the weight is defined by cohomological pairing, this is independent of Hamiltonian perturbations. Namely, $R_{j}=$ $\mathrm{wt}_{-\langle y\rangle, e_{\mathrm{p}}}\left(\tilde{u}_{j}\right)$ also, in the notation of the above lemma.

Finally, set

$$
\begin{gathered}
J_{(8)}^{\Lambda}=J_{(7)}^{\Lambda} ; \\
X_{(8)}^{\Lambda}=X_{(7)}^{\Lambda}+\sum_{n=1}^{\infty} \chi_{\tilde{H}_{[n]}^{\Lambda}}=\lim _{n \rightarrow \infty} \tilde{X}_{[n]}^{\Lambda} .
\end{gathered}
$$

Notice that the limit exists by (6.11). The limit of a sequence of regular $(J, X)$-homotopies might not be regular; however, (6.11) also implies:

$$
\left\|\sum_{j=n}^{\infty} \tilde{H}_{[j]}^{\Lambda}\right\|_{C_{\epsilon}} \leq \varepsilon_{R_{n}}\left(\tilde{J}_{[n]}^{\Lambda}, \tilde{X}_{[n]}^{\Lambda}\right) .
$$


Thus,

$$
X_{(8)}^{\Lambda}:=\tilde{X}_{[n]}^{\Lambda}+\chi_{\sum_{j=n}^{\infty} \tilde{H}_{[n]}^{\Lambda}}
$$

is $R_{n}$-regular, for any $n \in \mathbb{Z}^{+}$.

Depending on whether $\hat{\mathcal{M}}_{P}^{\Lambda, 0}\left(J_{(7)}^{\Lambda}, X_{(7)}^{\Lambda}\right)$ consists of finitely many points, either $n$ has a maximum, or $R_{n} \rightarrow \infty$. Either way, $\left(J_{(8)}^{\Lambda}, X_{(8)}^{\Lambda}\right)$ will be a regular $(J, X)$-homotopy: in the first case, $\left(J_{(8)}^{\Lambda}, X_{(8)}^{\Lambda}\right)=\left(\tilde{J}_{[n]}^{\Lambda}, \tilde{X}_{[n]}^{\Lambda}\right)$ for certain $n$, and is regular by definition; in the second case, the above argument shows that it is $R$-regular for all $R>0$.

$\left(J_{(8)}^{\Lambda}, X_{(8)}^{\Lambda}\right)$ is the admissible $(J, X)$-homotopy we desire. End of the proof of Proposition 6.2.2.

\section{References}

[1] A. Banyaga, Sur la structure du groupe des difféomorphismes qui préservent une forme symplectique, Comment. Math. Helv. 53(2) (1978), 174-227.

[2] M. Bershadsky, S. Cecotti, H. Ooguri and C. Vafa, Kodaira-Spencer theory of gravity and exact results for quantum string amplitudes, Commun. Math. Phys. 165 (1994), 311-427.

[3] D. Burghelea and S. Haller, Non-contractible periodic trajectories of symplectic vector fields. Floer cohomology and symplectic torsion, math.SG/0104013.

[4] K. Cieliebak, A. Floer and H. Hofer, Symplectic homology. II. A general construction, Math. Z. 218(1) (1995), 103-122.

[5] R. Cohen, J. Jones and G. Segal, Morse theory and classifying space, preprint available from http://math.stanford.edu/ ${ }^{\sim}$ ralph/ papers.html.

[6] S. Dostoglou and D. Salamon, Self-dual instantons and holomorphic curves, Ann. of Math. (2) 139(3) (1994), 581-640.

[7] T. Ekholm, J. Etnyre and M. Sullivan, Submanifolds in $R^{2 n+1}$ and contact homology, math.SG/0210124.

[8] Y. Eliashberg, Topological characterization of Stein manifolds of dimension > 2. Int. J. Math. 1(1) (1990), 29-46.

[9] Y. Eliashberg and M. Gromov, Lagrangian intersection theory: finitedimensional approach, in 'Geometry of differential equations', AMS, 1998.

[10] A. Floer, The unregularized gradient flow of the symplectic action. Commun. Pure Appl. Math. 41(6) (1988), 775-813. 
[11] A. Floer, Morse theory for Lagrangian intersections, J. Diff. Geom. 28(3) (1988), 513-547.

[12] A. Floer, Symplectic fixed points and holomorphic spheres. Commun. Math. Phys. 120(4) (1989), 575-611.

[13] A. Floer and H. Hofer, Coherent orientations for periodic orbit problems in symplectic geometry, Math. Z. 212(1) (1993), 13-38.

[14] A. Floer, H. Hofer and D. Salamon, Transversality in elliptic Morse theory for the symplectic action, Duke Math. J 80, (1995), 251.

[15] K. Fukaya, The symplectic s-cobordism conjecture: a summary, in 'Geometry and physics' (Aarhus, 1995), Dekker.

[16] K. Fukaya, Y. Oh, Zero-loop open strings in cotangent bundles and Morse homotopy, Asian J. Math. 1 (1998), 96.

[17] K. Fukaya and K. Ono, Arnold conjecture and Gromov-Witten invariant, Topology 38(5), (1999), 933-1048.

[18] Fukaya, Oh, Ohta, Ono, Lagrangian intersection Floer theoryAnomaly and obstruction (2000), preprint available from http://www.math.kyoto-u.ac.jp/ fukaya/fukaya.html.

[19] M. Gromov, Pseudo-holomorphic curves in symplectic manifolds, Invent. Math. 82(2) (1985), 307-347.

[20] H. Hofer and D. Salamon, Floer homology and Novikov rings, in 'The Floer memorial volume'. Progr. Math., 133, Birkhauser, Basel, 1995.

[21] M. Hutchings, Reidemeister torsion in generalized Morse theory. Forum Math. 14(2) (2002), 209-244.

[22] M. Hutchings and Y-J. Lee, Circle-valued Morse theory, Reidemeister torsion, and Seiberg-Witten invariants of 3-manifolds, Topology 38(4), (1999), 861-888.

[23] M. Hutchings and Y-J. Lee, Circle-valued Morse theory and Reidemeister torsion, Geom. Topol. 3 (1999), 369-396.

[24] K. Igusa, Higher Franz-Reidemeister torsion. AMS/IP Stud. Adv. Math., 31 (2002).

[25] E. Ionel and T. Parker, Gromov invariants and symplectic maps. Math. Ann. 314(1) (1999), 127-158.

[26] M. Kontsevitch, Homological algebra in mirror symmetry, in Proceedings of 1994, ICM.

[27] Y-J. Lee, Reidemeister torsion in symplectic Floer theory and counting pseudo-holomorphic tori II, J. Symplectic Geom., 3(3) (2006), 387-482.

[28] Y-J. Lee, Seiberg-Witten theory on 3-manifolds with euclidean ends, Commun. Anal. Geom., 13(1) (2005), 1-88. 
[29] Y-J. Lee and M. Sullivan, Reidemeister in the Floer of Lagrangian intersections, unfinished manuscript.

[30] Y-J. Lee, Noncontractible periodic orbits, Gromov invariants, and Floer-theoretic torsions, math.SG/0308185.

[31] Y-J. Lee, Corrected open Gromov invariants, in preparation.

[32] Le Hong Van and K. Ono, Symplectic fixed points, the Calabi invariant and Novikov homology, Topology 34(1) (1995), 155-176.

[33] G. Liu and G. Tian, Floer homology and Arnold conjecture, J. Diff. Geom. 49(1) (1998), 1-74.

[34] D. McDuff and D. Salamon, J-holomorphic curves and quantum cohomology, University Lecture Series, 6, AMS, 1994.

[35] J. Milnor, Whitehead torsion, Bull. Amer. Math. Soc. 72 (1966), $358-426$.

[36] J. Morgan and T. Mrowka, D. Ruberman, The $L^{2}$-moduli space and a vanishing theorem for Donaldson polynomial invariants, IP, 1994.

[37] S. Novikov, Multivalued functions and functionals. An analogue of the Morse theory. (Russian) Dokl. Akad. Nauk SSSR 260(1) (1981), 31-35.

[38] Y. Ruan, Virtual neighborhoods and pseudo-holomorphic curves. Proceedings of 6th G okova Geometry-Topology Conference, Turkish J. Math. 23(1) (1999), 161-231.

[39] D. Salamon, Lectures on Floer homology, in 'Symplectic geometry and topology'. (Park City, UT, 1997), AMS, 1999.

[40] D. Salamon and E. Zehnder, Morse Theory for Periodic Solutions of Hamiltonian Systems and the Maslov index, Commun. Pure Appl. Math, 17 (1992), 1303 .

[41] M. Schwarz, Morse homology, Birkhauser, 1993.

[42] P. Seidel, Symplectic Floer homology and the mapping class group, Pacific J. Math. 206(1) (2002), 219-229.

[43] M. Sullivan, K-theoretic invariants for Floer homology. Geom. Funct. Anal. 12(4) (2002), 810-872.

[44] C.H. Taubes, Counting Pseudo-holomorphic submanifolds in dimension 4, J. Diff. Geom., 44(4) (1996), 818-893.

[45] V. Turaev, Torsion invariants of Spin ${ }^{c}$-structures on 3-manifolds. Math. Res. Lett. 4(5) (1997), 679-695.

[46] C. Viterbo, Functors and computations in Floer homology with applications. I. Geom. Funct. Anal. 9(5) (1999), 985-1033. 
Department of Mathematics

Purdue University

WEST LAFAYETTE, IN 47907

E-mail address: yjlee@math.purdue.edu

This work began in 1997 as a joint project with Michael Hutchings. The author is indebted to M.H. for his early input during 1997-1998; especially, the finite-dimensional picture he worked out in [21] was indispensable. She also thanks P. Seidel for suggesting this problem, M. Sullivan for explaining his related work, D. Burghelea for communicating their relevant preprint when this work was first circulated in 2001. The author was supported by MSRI, IAS, and NSF grant DMS \# 0333163 during the multiple re-writings of this work. 
